\title{
PHYSICS AT THE MEV-SCALE IN LIQUID ARGON TIME PROJECTION CHAMBERS
}

\section{BY}

IVAN THOMAS LEPETIC

Submitted in partial fulfillment of the requirements for the degree of Doctor of Philosophy in Department of Physics in the Graduate College of the Illinois Institute of Technology

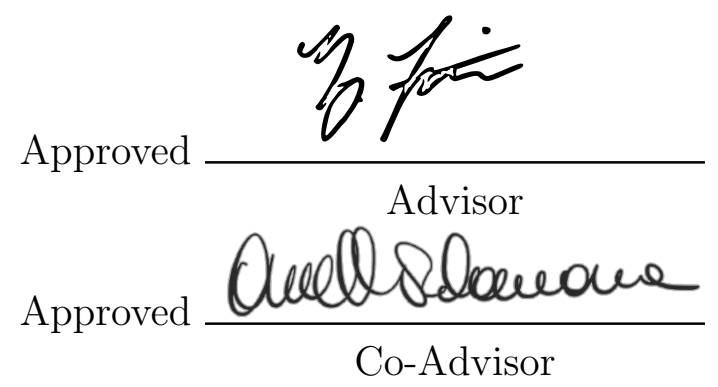

Chicago, Illinois

May 2020 



\section{ACKNOWLEDGMENT}

I would like to thank my advisor Bryce Littlejohn for his constant and unwavering support during graduate school. From the very beginning, he patiently guided me in the right direction, pointing to paths I could not envision at the time. His encouragement and guidance during many challenging moments were indispensable for reaching the point where I am now. Writing a paper, getting a grant and awards to support my studies would not have been possible without his help. He entrusted in me the responsibility to mentor several undergraduates, so I was able to mimic some of his knowledge and generosity to others.

I was also very fortunate to have Ornella Palamara as my Fermilab advisor. Her knowledge and energy were not only challenging but inspiring too. Her advice and guidance went way above and beyond the ordinary. She spent countless hours teaching me about particle physics and the minutiae of scientific analysis. It is impossible to appropriately express the appreciation and gratitude I feel for Ornella's personal and friendly touch to all the work we have done together.

Among many other people whose invaluable help made my graduate work easier and often fun, I particularly want to thank Flavio Cavanna and Roni Harnik. Although their vast knowledge of physics was often intimidating, I immensely profited from their input and advice. It was a pleasure to work with Zhen Liu on the millicharged particle search. Jonathan Asaadi introduced me to Fermilab and helped me get started. Paola Sala was generous with her time, making my stay at CERN productive and pleasant. Tingjun Yang answered the many questions I had when I began working on ArgoNeuT.

Matt, Bishu, Jyoti, Steven and Connor have been wonderful colleagues and officemates. Our conversations were a nice respite. I thank them all. 
I want to thank Yudong for her encouragement and love. Finally, I want to thank my parents for their unwavering love and support throughout my life. 
TABLE OF CONTENTS

Page

ACKNOWLEDGEMENT . . . . . . . . . . . . . . . iii

LIST OF TABLES . . . . . . . . . . . . . . . . . . vii

LIST OF FIGURES . . . . . . . . . . . . . . . . . . . . . xiii

LIST OF ABREVIATIONS . . . . . . . . . . . . . . . xiv

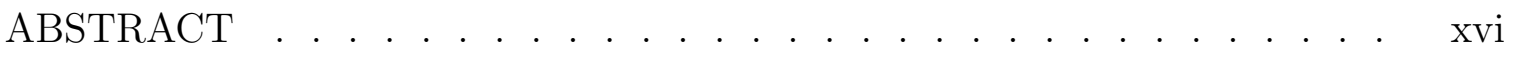

CHAPTER

1. EXECUTIVE SUMMARY . . . . . . . . . . . . . . . 1

2. THE STANDARD MODEL, NEUTRINOS AND BEYOND . . . 3

2.1. The Standard Model . . . . . . . . . . . . . . . . . . 3

2.2. History of Neutrinos . . . . . . . . . . . . . . . . . . 6

2.3. Sources of Neutrinos . . . . . . . . . . . . . . . . . . 10

2.4. Neutrino Interactions . . . . . . . . . . . . . . . . . . . 21

2.5. Neutrino Oscillations . . . . . . . . . . . . . . . . . 25

2.6. Neutrino Mass . . . . . . . . . . . . . . . . . . . . . . 30

2.7. Neutrino Detection . . . . . . . . . . . . . . 31

2.8. Beyond the Standard Model and Millicharged Particles . . 31

3. THE LIQUID ARGON TIME PROJECTION CHAMBER . . . 37

3.1. Principle of Operation . . . . . . . . . . . . . . . . 39

3.2. Particle Propagation Through Argon and Detection . . . . 41

3.3. The ArgoNeuT LArTPC Experiment at Fermilab . . . . . 46

3.4. Other LArTPC Neutrino Experiments at Fermilab . . . . . 52

3.5. Event Reconstruction in LArTPCs . . . . . . . . . 55

4. LARTPC PHYSICS STUDIES . . . . . . . . . . . . . . . 62

4.1. Neutrino Physics Studies at GeV Scales . . . . . . . . 62

4.2. Benefits of MeV-Scale Abilities for GeV-Scale Physics Studies 63

4.3. Neutrino Physics Studies at MeV Scales . . . . . . . . . 66 
4.4. BSM Physics Studies and MeV-Scale Capabilities . . . . . 70

5. FIRST DEMONSTRATION OF MEV-SCALE PHYSICS IN LIQUID ARGON TIME PROJECTION CHAMBERS USING ARGONEUT . . . . . . . . . . . . . . . . . . . . . 72

5.1. MeV Scales in LArTPCs . . . . . . . . . . . . . . . . 72

5.2. Production and Interaction of Low-Energy Photons in NeutrinoArgon Interactions . . . . . . . . . . . . . . . . . . . . 73

5.3. Datasets . . . . . . . . . . . . . . . . . 74

5.4. Event Reconstruction . . . . . . . . . . . . . . . . . . 78

5.5. Results . . . . . . . . . . . . . . . . . . 86

5.6. Conclusions . . . . . . . . . . . . . . . . . . . . . . . 93

6. MILLICHARGED PARTICLE SEARCH USING ARGONEUT . 95

6.1. Production of Millicharged Particles Reaching ArgoNeuT . 95

6.2. Detection Signature . . . . . . . . . . . . . . 96

6.3. Datasets . . . . . . . . . . . . . . . . . . . 97

6.4. Reconstruction . . . . . . . . . . . . . . . . . . . . . 98

6.5. High-Beam vs Low-Beam Data . . . . . . . . . . . . . . 99

6.6. Analysis . . . . . . . . . . . . . . . . . . . . . . . . . 99

6.7. Expected Signal and Background . . . . . . . . . . . . . 102

6.8. Search for mCP Candidates . . . . . . . . . . . . . . . 104

6.9. Summary . . . . . . . . . . . . . . . . 106

7. CONCLUSIONS . . . . . . . . . . . . . . . . . . . . . 109

BIBLIOGRAPHY . . . . . . . . . . . . . . . . . . . . . . . 110 


\section{LIST OF TABLES}

Table

Page

2.1 Masses and Charges of Particles in the Standard Model. Neutrinos are treated as massless in the SM. Charge is given in units of the charge of the electron. Values from [1]. . . . . . . . . . . . . . . 35

2.2 Generations of the Standard Model. From [2] . . . . . . . . . . . 36

2.3 Current best-fit values for neutrino mixing angles, mass splittings and $\mathrm{CP}$ violating phase. From [1]. . . . . . . . . . . . . . . . . . . 36

4.1 Expected interaction rates in DUNE of neutrinos from a supernova 10 kpc away. Livermore model does not assume oscillations, but GKVM assumes collective oscillations. From [3]. . . . . . . . . . . . .

5.1 Effectiveness of different cuts for collection plane hits. Cuts are applied sequentially. There are 552 neutrino, 1970 background and 3889 $\mathrm{MC}$ events. . . . . . . . . . . . . . . . . . . . . . . . . . . . .

5.2 Comparison of neutrino and background datasets when examining hits passing all cuts. The difference in the first four metrics indicates neutrino-induced $\mathrm{MeV}$-scale activity is visible. . . . . . . . . . . .

5.3 Relative contributions of de-excitation and neutron-produced photon components in FLUKA MC. . . . . . . . . . . . . . . . . . .

6.1 Number of plane matched clusters passing cuts in an event for events with low-beam intensity ("low-beam") and events without high-energy activity ("high-beam"). . . . . . . . . . . . . . . . . . . 


\section{LIST OF FIGURES}

Figure

Page

2.1 The Standard Model of particle physics. Image from [4]. . . . . .

2.2 The sources and energies of neutrinos. The $\mathrm{x}$-axis indicates the energy of the neutrino. The y-axis indicates the cross section of $\bar{\nu}_{e}-e$ scattering. Image from $[5] .$. . . . . . . . . . . . . . . . .

2.3 An illustration of the effect on the paths of charged hadrons from magnetic focusing horns. The proton beam enters from the left, striking the target and producing hadrons. The vertical scale is four times the horizontal scale. Image from [6]. . . . . . . . . . . . .

2.4 Diagram of infrastructure for the NuMI beam. Image from [6]. . .

2.5 Flux of neutrinos from the NuMI beam with different horn settings. Perfect focusing refers to every pion being perfectly focused along the beam axis. The y-axis indicates the event rate at the MINOS far detector; it is included here to describe the relative fluxes. Image

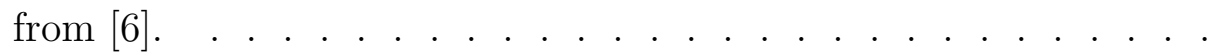

2.6 Flux of neutrinos from the NuMI beam operating in the low-energy, antineutrino mode and entering ArgoNeuT, a liquid argon detector, $1 \mathrm{~km}$ downstream. Image from [7]. . . . . . . . . . . . . . . . .

2.7 Flux of neutrinos from the BNB beam at MiniBooNE. The flux is roughly the same for all BNB experiments. Image from [8]. . . . .

2.8 Solar neutrino flux spectrum listing the sources mentioned in the

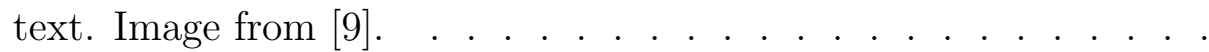

2.9 Supernova neutrino spectrum. The dip in the $\nu_{e}$ spectrum is due to collective effects. Note that oscillation effects in such a dense medium are not fully understood and could substantially alter the spectrum. Image from $[10]$. . . . . . . . . . . . . . . . . . .

2.10 Relative event times and energies of neutrinos from SN1987A. Image from [11]. . . . . . . . . . . . . . . . . . .

2.11 Charged current (left) and neutral current (right) neutrino interactions on neutrons. Note that the neutral current reaction produces no charged lepton. . . . . . . . . . . . . . . . . . . . . . . . .

2.12 Measurements of cross sections for quasielastic scattering, resonance production and deep inelastic scattering for neutrinos. From [5]. . 
2.13 Feynman diagram of a pair of millicharged particles produced by the decay of a $\pi^{0}$ meson. . . . . . . . . . . . . . . . .

3.1 Diagram of LArTPC operation. As charged particles travel through the liquid argon, electrons are ionized. A cathode (left) biased at a high voltage creates an electric field which causes the ionized electrons to drift to planes of wires (middle) which detect the electrons. Wave forms on wires are read out, and the activity of the charged particles is reconstructed (right). No light collection system is shown here, however it is typically placed behind the wire planes. Image

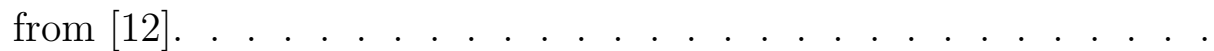

3.2 Cross-sections of photon interactions in liquid argon for energies between $0.1-10 \mathrm{MeV}$. Data from [13]. . . . . . . . . . . . . .

3.3 Cross-sections for inelastic scattering with photon production (gold square), proton emission (blue circle), and neutron emission (red triangles and gray diamonds). Image from [14]. . . . . . . . . . .

3.4 Energies of individual photons from $100 \mathrm{keV}$ neutrons (left) and 10 $\mathrm{MeV}$ neutrons (right). Note the peak at $1.46 \mathrm{MeV}$ due to the first excited state of ${ }^{40} \mathrm{Ar}$ in the right plot. . . . . . . . . . . . .

3.5 Total energy of photons from $100 \mathrm{keV}$ neutrons (left) and $10 \mathrm{MeV}$ neutrons (right). The left plot shows photons only from neutron capture, while the right plot shows photons from neutron capture and inelastic scattering. . . . . . . . . . . . . . .

3.6 Image of the ArgoNeuT TPC (front) and cryostat (back). The wires are seen on the right of the TPC. Image from [15]. . . . . . . . .

3.7 Diagram of the ArgoNeuT TPC showing the layout of the different TPC components. The inset shows the cathoe and field shaping rectangular rings. Image from $[15] \ldots$. . . . . . . . . . .

3.8 ArgoNeuT's coordinate system and wire planes. Image from [15]. .

3.9 Relative locations of ArgoNeuT and the MINOS ND. The neutrino

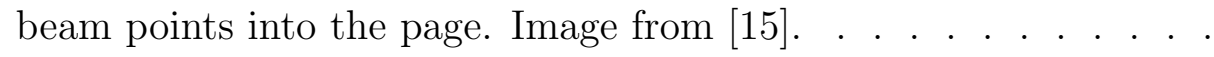

3.10 Matching of tracks between ArgoNeuT and the MINOS ND. A muon (long track) produced in ArgoNeuT (small box in foreground) leaves ArgoNeuT and enters the MINOS ND (large octagon in background). Also shown are other muons produced within the MINOS ND. Note the curvature of the muon tracks due to the magnetic field within the MINOS ND. Image from [15]. . . . . . . . . . . 
3.11 Wire signals from an ArgoNeuT event. Top plane is induction, bottom collection. The horizontal axis indicates time sample number, and the vertical axis indicates signal strength. Raw wire signals are in black (note the bipolar form of the raw signal in the induction plane), deconvoluted wire signals in blue, and fitted peaks in red. .

3.12 Tracks identified by the automated reconstruction in an ArgoNeuT neutrino event. Color indicates the two different tracks. Image

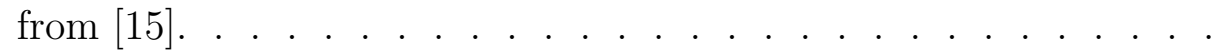

3.13 The same event as in Figure 3.12, reconstructed in 3D. Note that the tracks exit the detector. Image from $[15] \ldots$. . . . . . . . .

3.14 Recombination factor as a function of $d E / d x$ in an electric field of $480 \mathrm{~V} / \mathrm{cm}$ for a variety of models. ArgoNeuT and other LArTPCs currently use the Modified Box Model. Image from [16]. . . . . .

3.15 Amount of energy deposited per unit length $(d E / d x)$ versus residual range for a variety of particles in liquid argon. Image from [15]. . .

4.1 An electron neutrino CC event in ArgoNeuT displaying a highenergy electron, seen as a shower. Other hadronic activity is seen at the vertex. Only the collection plane is shown Wire number is indicated on the x-axis. The y-axis indicates time in ticks. Color indicates amount of charge collected. Image from [7]. . . . . . . .

4.2 An muon neutrino $\mathrm{CC}$ event in ArgoNeuT. The muon is seen as the long, nearly horizontal track. This event display also shows two protons traveling away from the vertex back-to-back, indicating nuclear effects. Top plane is collection, bottom induction. Wire number is indicated on the $\mathrm{x}$-axis. The $\mathrm{y}$-axis indicates time in ticks. Color indicates amount of charge collected. Image from [17].

4.3 Energy spectrum of neutrons as predicted by three neutrino interaction generators for $3 \mathrm{GeV}$ antineutrinos interacting on polystyrene. This energy is typical of beam neutrinos. While there is disagreement between the generators, all three indicate that a substantial portion of the antineutrino's energy can be given to neutrons. Image from $[18] \ldots \ldots \ldots \ldots$

4.4 Energy spectrum of Michel electrons from a simulation of cosmic rays in MicroBooNE. Red denotes the true Michel electron spectrum. Black denotes the reconstructed spectrum using only energy deposited by ionization; this excludes energy lost in the form of radiative photons. Distributions are area normalized. From [12]. . 
4.5 A simulated supernova neutrino CC absorption event in ArgoNeuT. A short electron track is seen along with activity due to deexcitation photons. Top plane is collection, bottom induction. Wire number is indicated on the $\mathrm{x}$-axis. The $\mathrm{y}$-axis indicates time in ticks. Color indicates amount of charge collected. . . . . . . . . . . . . . . .

4.6 Present (left) and future (right) measurements of neutrino mixing parameters with solar and reactor neutrinos. Curves indicate 1, 2 and $3 \sigma$ confidence intervals. DUNE will be able to significantly improve these values by reconstructing solar neutrinos. From [19].

5.1 Energy (top) and multiplicity (bottom) of low-energy photons from charged current interactions of muon neutrinos from the NuMI beam interacting and depositing energy in a volume of liquid argon with the dimensions of ArgoNeuT. Color indicates source of photon (red are de-excitation photons, blue are photons produced by neutrons). For a photon to be tracked in the simulation, it must have an energy $\geq 0.2 \mathrm{MeV}$. . . . . . . . . . . . . . . . . . . . . . . . .

5.2 A neutrino event (raw data) with one track reconstructed as a muon and one track reconstructed as a proton. Possible gamma activity (isolated blips) is visible around the muon track. Top plane is collection, bottom induction. Wire number is indicated on the x-axis. The y-axis indicates time in ticks. Color indicates amount of charge collected. . . . . . . . . . . . . . . . . .

5.3 Energy vs range for electrons and protons for the ranges of interest for this study. Red denotes protons, blue denotes electrons. The clear separation between electron and proton means it is unlikely a proton hit will be mistakenly identified as an electron hit. Data from $[20]$. . . . . . . . . . . . . . . . . . . . . . . . .

5.4 A raw neutrino data event with one track reconstructed as a muon and with gamma activity (isolated blips). Top plane is collection, bottom induction. Wire number is indicated on the $\mathrm{x}$-axis. The $\mathrm{y}$-axis indicates time in ticks. Color indicates amount of charge collected. . . . . . . . . . . . . . . . . . . . .

5.5 The same event as in Figure 5.4 after hit finding and reconstruction. Each square denotes a reconstructed hit. Color indicates whether or not a hit was removed and by which cut (see text). Hits that pass all cuts are in red. . . . . . . . . . . . . . . . . . . . .

5.6 Energy deposited vs collected charge. Line indicates fit used to perform energy calculations from collected charge. . . . . . . . . 
5.7 Reconstructed energy vs true electron energy using the charge method for a sample of simulated electrons with energies between 0 and 5 $\mathrm{MeV}$. Events where the electron was not detectable are excluded. .

5.8 Energy deposited in individual one-hit clusters according to the FLUKA simulation. . . . . . . . . . . . . . . . . . . . . . . .

5.9 Cluster multiplicity (top) and total signal reconstructed energy (bottom) in an event for neutrino data and FLUKA MC events. Events with no reconstructed energy are not included. Data points include statistical error. Dark green line indicates FLUKA prediction with data-driven background added (see text). Dark green shaded area is statistical error in FLUKA, overlaid on total error (statistical + systematic) for FLUKA in light green shading. MC is normalized to the number of neutrino data events. . . . . . . . . . . . . . .

5.10 Cluster multiplicity (top) and total signal reconstructed energy (bottom) in an event for neutrino data and GENIE MC events. Events with no reconstructed energy are not included. Data points include statistical error. Dark blue line indicates GENIE prediction. Light blue shaded area indicates statistical error for GENIE prediction. $\mathrm{MC}$ is normalized to the number of neutrino data events. . . . . .

5.11 Cluster multiplicity (left) and total signal reconstructed energy (right) in an event for neutrino data and FLUKA MC events. Events with no reconstructed energy are not included. Data points include statistical error. Dark green indicates FLUKA prediction with only deexcitation photons (top) or with only neutron-produced photons (bottom). Data-driven background is added to all plots (see text). $\mathrm{MC}$ is normalized to the number of neutrino data events. . . . . .

5.12 Distributions of cluster position with respect to the neutrino interaction vertex in neutrino data and FLUKA MC events. Data includes statistical error. Green indicates the contribution of photons from de-excitation of the final-state nucleus. Red indicates the contribution of photons from inelastic neutron scattering. $\mathrm{MC}$ is area normalized to data. . . . . . . . . . . . . . . . . . . . .

6.1 Number of millicharged particles with charge $10^{-2} e$ entering ArgoNeuT for $10^{20} \mathrm{POT}$, integrated over all millicharged particle energies. Colors indicate which neutral meson decay. Yellow dot-dash indicates Drell-Yan production. From [21]. . . . . . . . . . . . 
6.2 Schematic (not to scale) of the ArgoNeuT detector location relative to the upstream target. Signal is a double-hit event with a line defined by the two hits pointing to the target (top). A background double-hit event generally will not point to the target (bottom). Figure adapted from Ref. [21]. . . . . . . . . . . . . . . . . . .

6.3 Top left: Energy deposited in each cluster in high-beam events with at least two clusters. Top right: Distance between clusters in a line in events with two or more clusters. Bottom left: Energy deposited in individual one-hit clusters for high beam events. The majority (97\%) are below 2.5 MeV. Bottom right: Cosine of angle between line and vector pointing to the target. Any $\mathrm{mCP}$ signal should appear at $\cos \theta=1$. . . . . . . . . . . . . . . . . . . . . . .

6.4 Locations of the points of intersections (shown as grey circles) of lines defined by two clusters with a plane perpendicular to the beam at the downstream target's edge. Uncertainties in the location (see text) are also shown. The target, denoted by the red cross, is located at $(0,0)$. The candidate signal event, denoted with a blue square, is consistent with originating from the target within its uncertainties. Note that the scale in the vertical axis is $10 \times$ that of the horizontal axis, since the horizontal uncertainties are smaller. Only points at a distance $<10(100) \mathrm{m}$ from the target in the horizontal (vertical) direction are shown. . . . . . . . . . . . . . . . . . . . .

6.5 The candidate signal event. Top: Zoomed in image from the collection wire plane. Two isolated clusters are visible in the event. The two clusters are spaced $11.8 \mathrm{~cm}$ apart with an energy of 0.72 (2.82) $\mathrm{MeV}$ in the left (right) cluster. Color in the image indicates the amount of charge collected. The horizontal axis is perpendicular to the collection plane wires. The vertical axis is parallel to the drift direction. Bottom: 3D reconstruction of the event with the reconstructed line superimposed. . . . . . . . . . . . . . . . .

6.6 ArgoNeuT limits (blue) in the $m_{\chi}-\epsilon$ plane for millicharged particles at $95 \%$ C.L., where $\epsilon \equiv Q_{\chi} / e$. The limit is drawn where mCP are unlikely to produce more than the observed number of events. The thickness of the blue band accounts for the systematic uncertainty in detector placement. Existing limits from other experiments, including SLAC MilliQ [22] and collider experiments [23-26], are shown in gray. 


\section{LIST OF ABBREVIATIONS}

Abbreviation/Symbol

ADC

BNB

BSM

CC

CM

$\mathrm{CNO}$

CRT

DONUT

DUNE

$\mathrm{eV}$

$\mathrm{IH}$

LArTPC

$\mathrm{mCP}$

MINOS

$\mathrm{NC}$

ND

$\mathrm{NH}$

NuMI

PMNS

PMT

POT
Definition

Analog to Digital Conversion

Booster Neutrino Beam

Beyond the Standard Model

Charged Current

Center of Momentum

Carbon-Nitrogen-Oxygen

Cosmic Ray Tagger

Direct Observation of the $\mathrm{Nu}$ Tau

Deep Underground Neutrino Experiment

Electron Volt

Inverted Hierarchy

Liquid Argon Time Projection Chamber

MilliCharged Particle

Main Injector Neutrino Oscillation Search

Neutral Current

Near Detector

Normal Hierarchy

Neutrinos at the Main Injector

Pontecorvo-Maki-Nakagawa-Sakata

PhotoMultiplier Tube

Protons on Target

xiv 


$\begin{array}{cl}\text { PP } & \text { Proton-Proton } \\ \text { QE } & \text { Quasi-Elastic } \\ \text { SBND } & \text { Short Baseline Near Detector } \\ \text { SiPM } & \text { Silicon PhotoMultiplier } \\ \text { SM } & \text { Standard Model } \\ \text { SNO } & \text { Sudbury Neutrino Observatory } \\ \text { Super-K } & \text { Super Kamiokande } \\ \text { VUV } & \text { Vacuum UltraViolet }\end{array}$




\begin{abstract}
The liquid argon time projection chamber (LArTPC) is the detection technology chosen for several Fermilab-based neutrino experiments. This technology will be used in studies of neutrino cross-sections and oscillations, neutrinos from supernovae as well as a variety of studies of beyond the Standard Model physics. This thesis explores the use of these detectors to study MeV-scale activity. MeV-scale electrons arising from Compton scatters of deexcitation photons and photons from inelastic neutron scattering in neutrino-nucleus interactions are reconstructed using novel methods presented here. This work represents the first demonstration of $\mathrm{MeV}$ scale physics capabilities in a LArTPC neutrino experiment as well as the first observation of neutrino-produced photons from nuclear de-excitation and inelastic neutron scattering. A search for millicharged particles, postulated by theories of beyond the standard model physics, is also performed using data from a LArTPC and the lowenergy reconstruction techniques developed. The results set world-leading bounds on the parameter space of millicharged particles. The work in this thesis demonstrates that studies of MeV-scale activity and new physics are possible with LArTPC technology and provides the foundation for future LArTPC studies of low energy neutrinos and new physics.
\end{abstract}




\section{CHAPTER 1 \\ EXECUTIVE SUMMARY}

The upcoming Deep Underground Neutrino Experiment (DUNE) [27] and the SBN Program [28] seek to explore a variety of phenomena in the universe, from the properties of neutrinos, to supernovae, to physics beyond our current models. To do this, DUNE and SBN will use one of the most powerful detector technologies available, the liquid argon time projection chamber (LArTPC). This technology has proven its value for a variety of physics studies, particularly using $\mathrm{GeV}$-scale beam neutrinos, but questions remain. LArTPCs have not been used to study activity at the MeV-scale. They have not been used to examine neutrons or low-energy photons. In fact, until the studies presented here, it was not clear that these detectors are even able to see activity from these neutral particles. DUNE wishes to study supernova and possibly even solar neutrinos, but these neutrinos are low-energy, and no lowenergy neutrinos have been detected in LArTPCs. Proper reconstruction of such neutrinos is dependent on the ability to reconstruct low-energy activity, far below the typical energies of beam neutrinos. DUNE and SBN also seek to test theories of new, beyond the standard model (BSM) physics, but no such studies have been performed in LArTPCs.

This thesis presents the first demonstration of MeV-scale physics in LArTPCs by accurately identifying and reconstructing activity due to low-energy photons from neutrino-produced nuclear deexcitation and inelastic neutron scattering. This work is the first reported detection of deexcitation photons and photons from inelastic scattering of final-state neutrons from beam neutrino interactions in argon. The potential detection and reconstruction of activity from neutrino-produced neutrons is barely addressed in the literature, but is of utmost importance for a wide variety of physics 
goals, for example those examining the difference of final state neutron production in neutrino versus antineutrino interactions. By reconstructing the multiplicity and energy of neutrons emitted in a neutrino interaction, it may be possible to determine whether a neutrino or antineutrino interacted.

This work also presents the first search for beyond the standard model physics in a LArTPC. Using the techniques developed in the first part of this work, a search for millicharged particles is carried out with ArgoNeuT data. Millicharged particles have been proposed as a constituent of dark matter and may resolve an astrophysical experimental anomaly. This search places world-leading limits on the parameter space of millicharged particles and demonstrates the potential for LArTPC technology to search for new physics.

Both studies in this thesis were performed using ArgoNeuT, a small (quarterton) LArTPC. ArgoNeuT collected data in the Neutrinos at the Main Injector beam at Fermilab for a short period, less than six months, yet the work presented here is competitive with much larger and longer-running detectors. This proves not just the usefulness of the low-energy techniques presented here, but also the potential of this detection technology as a whole. The work here also sets the stage for and makes possible a variety of physics studies to be performed in the Short Baseline Program [28] and DUNE [3]. 
CHAPTER 2

\section{THE STANDARD MODEL, NEUTRINOS AND BEYOND}

This thesis focuses on reconstructing MeV-scale activity in liquid argon time projection chambers. This detection technology is typically used in neutrino experiments but may also be used for studies of physics beyond the scope of the current theory of particles, called the Standard Model. This chapter presents an introduction to the Standard Model and discusses neutrinos and their role in the Standard Model. This chapter also discusses sources of neutrinos, particularly particle accelerators, the sun and supernovae which span the sub-MeV to multi-GeV energy regime. Studies of neutrinos from all three of these sources would benefit from the work presented later in this thesis. This chapter ends by examining additional physics beyond the Standard Model, with an introduction to millicharged particles.

\subsection{The Standard Model}

The theory describing the known elementary particles and the strong, weak and electromagnetic forces is called the Standard Model (SM). The SM consists of eighteen particles: twelve fermions, which have spin $1 / 2$, and six bosons which have integer spin. The forces are mediated by five of the six bosons. The strong force is mediated by the gluon $(g)$, the electromagnetic force by the photon $(\gamma)$ and the weak force by the charged $W^{+}$and $W^{-}$bosons and neutral $Z$ boson. Finally, the Higgs $(H)$ boson gives the massive particles their mass. ${ }^{1}$

The SM is based on a local symmetry group

$$
\mathrm{SU}(3)_{C} \times \mathrm{SU}(2)_{L} \times \mathrm{U}(1)_{Y}
$$

${ }^{1}$ See Sec. 2.6. 
where $C$ denotes color, $L$ denotes left-handed chirality and $Y$ denotes hypercharge. The theory of strong interactions is based on the $\mathrm{SU}(3)_{C}$ group and is called quantum chromodynamics. While the strong interaction can be separated from the the other two interactions, the electromagnetic and weak interactions cannot be separated, and together are called electroweak. Electroweak interactions are determined by the latter two groups of Eq. 2.1, $\mathrm{SU}(2)_{L} \times \mathrm{U}(1)_{Y}$.

The SM symmetry group (Eq. 2.1) sets the number of vector gauge bosons based on the number of generators for each group. There are eight generators for $\mathrm{SU}(3)_{C}$, thus there are eight gluons. There are three generators of $\mathrm{SU}(2)_{L}$, the weak force mediators $W^{ \pm}$and $Z$, and one $\mathrm{U}(1)_{Y}$ mediator, the photon. While the number of vector gauge bosons is set by the SM, the number of scalar bosons and fermions is not.

Fermions make up the remainder of the SM and are further divided into two types: quarks and leptons (see Fig. 2.1). The six quarks, $u$ (up), $d$ (down), $c$ (charm), $s$ (strange), $t$ (top) and $b$ (bottom) feel the strong, weak and electromagnetic forces. Three of the leptons, $e$ (electron), $\mu$ (muon), $\tau$ (tau), feel the weak and electromagnetic force, and the remaining three leptons, the neutrinos, feel only the weak force. The masses and charges of the fermions are given in Table 2.1. All of the fermions can be grouped into three generations, shown in Table. 2.2 which are similar except for their mass, which increases with generation number. Along with every particle in the SM, there is a corresponding antiparticle. For charged particles, the antiparticle has the same mass but opposite charge. For neutral particles, the antiparticle may be the same particle, as with the photon, or it may be a different particle.

Neutrinos are fundamental particles that are abundant, light, electrically neutral and come in three flavors. They are the second most common particle in the universe, after photons, and are produced from a variety of sources, both natural and 


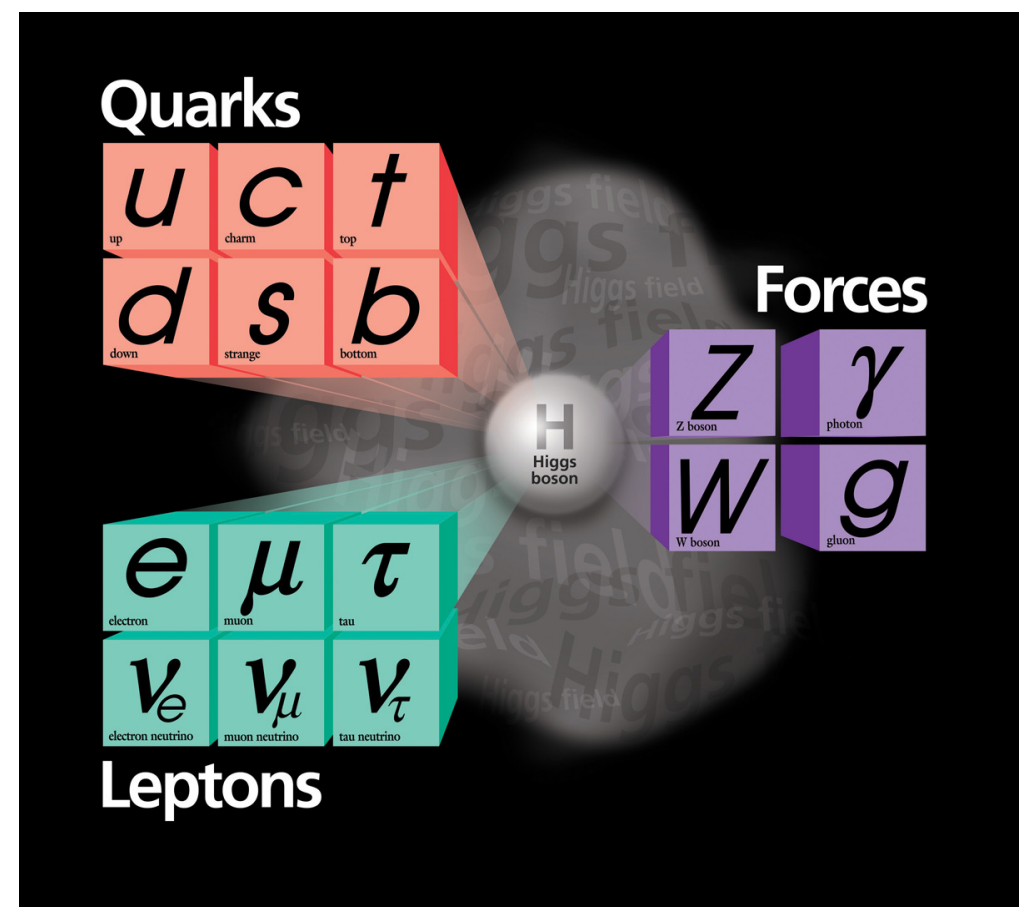

Figure 2.1. The Standard Model of particle physics. Image from [4].

man-made (see Sec. 2.3). Neutrinos are light in that their mass states have a mass many orders of magnitude smaller than all the other massive fundamental particles in the Standard Model.

Because they are electrically neutral, neutrinos do not feel the electromagnetic force. As leptons, they do not couple to the strong interaction, but do couple to the weak interaction. Because of this, detecting a neutrino is very difficult. There are three types, or flavors of neutrinos, electron $\left(\nu_{e}\right)$, muon $\left(\nu_{\mu}\right)$ and tau $\left(\nu_{\tau}\right)$, and each neutrino flavor is associated with a corresponding charged lepton, the electron, muon and tau. For each of the three neutrinos, there is also a corresponding antineutrino, but it is an unresolved question whether neutrinos are their own antiparticles.

The SM sets the masses of the neutrinos at zero, thereby fixing its flavor. It is now known that neutrinos do have mass, a discovery made by the observation of neutrino oscillations, but it is unclear where their mass comes from. A neutrino's 
flavor will change as it travels through space, a phenomenon called oscillation, and the study of this oscillation is a major topic of neutrino research today [29].

\subsection{History of Neutrinos}

The neutrino was postulated by Pauli in 1930 as the solution to a problem posed by beta decay. In beta decay, a heavier nucleus decays into a lighter one along with the emission of an electron and (as we now know) an electron antineutrino. Without the presence of the antineutrino, beta decay is simply a two-body decay $A \rightarrow B+e$. As with any two-body decay, in the center of momentum (CM) frame, the original nucleus is at rest, and the products fly off with momenta of equal magnitude but opposite direction. The electron's energy is then a constant given by:

$$
E_{e}=\left(\frac{m_{A}^{2}-m_{B}^{2}+m_{e}^{2}}{2 m_{A}}\right) c^{2}
$$

where $m_{A}$ is the mass of nucleus $A, m_{B}$ is the mass of nucleus $B, m_{e}$ is the electron mass, and $c$ is the speed of light. Experiments showed this energy is not a constant; instead there is a spectrum of electron energies. Pauli proposed there was a third, undetected particle, which took away some of the energy. Pauli argued his new particle must be electrically neutral, have spin $1 / 2$ and have a mass less than $1 \%$ the mass of the proton. Fermi later incorporated this particle into his theory of beta decay [30] and called it the neutrino. In Fermi's theory, a neutron turns into a proton and creates an electron and an electron antineutrino. (The neutron had not been discovered when beta decay was first observed.) This theory also indicates how a neutron would interact with matter. Fermi's theory correctly explained the shape of the beta decay spectrum but incorrectly gave the neutrino a mass of zero [2].

The existence of the electron antineutrino was confirmed by Cowan and Reines in 1956 [31]. The experiment relied on the Savannah River nuclear reactor as a source 
of electron antineutrinos, which were detected via the inverse of beta decay:

$$
\bar{\nu}_{e}+p \rightarrow n+e^{+}
$$

The detector was a tank of water and $\mathrm{CdCl}_{2}$. The experimental observable was two 0.511 MeV photons from the annihilation of the positron with an electron along with a neutron capture on the Cadmium occurring about a few microseconds later and also producing photons. This detection technique is still used for reactor neutrino experiments [2].

Around this time, the question of whether neutrinos and antineutrinos are the same particle was posed. Rearranging Eq. 2.3 yields a crossed reaction $\nu_{e}+n \rightarrow$ $p+e^{-}$. If neutrinos and antineutrinos are the same particle, then one could replace the electron neutrino with its counterpart and get

$$
\bar{\nu}_{e}+n \rightarrow p+e^{-}
$$

however this reaction does not occur in experiments. Consequently, the idea of conservation of lepton number was introduced. All matter leptons are given a lepton number of +1 , and all antimatter leptons are given a lepton number of -1 . Other particles have a lepton number of zero. Furthermore, experiments indicated the reaction $\mu^{-} \rightarrow e^{-}+\gamma$ does not occur, so it is believed that lepton number is conserved within flavors as well [2].

The existence of a second type of neutrino was proposed as an answer to another three-body decay, this time the decay of the muon $\mu^{-} \rightarrow e^{-}+\bar{\nu}_{e}+\nu_{\mu}$. Experiments showed that the energy of the electron was not a constant, again implying a three body decay. Because lepton number is conserved within each flavor, the reaction $\nu_{\mu}+n \rightarrow p+e^{-}$cannot occur. Pontecorvo then suggested that by using charged pion decays, which produce almost no electron neutrinos or antineutrinos, but do produce muon (anti)neutrinos, one could prove that $\nu_{\mu}$ and $\nu_{e}$ are two different neutrinos. In 
1962, Lederman, Schwartz and Steinberger [32] conducted an experiment using muon neutrinos from the AGS particle accelerator at Brookhaven National Laboratory and observed the reaction

$$
\nu_{\mu}+n \rightarrow p+\mu^{-}
$$

providing the first evidence for two types of neutrinos [2].

As will be discussed in Sec. 2.4, neutrinos interact via the weak force, the theory of which was developed by Glashow, Weinberg and Salam in the 1960s. Their theory proposed the existence of two gauge bosons, the $\mathrm{W}$ and the $\mathrm{Z}$. Interactions by the $\mathrm{W}$ boson, called charged current interactions had already been observed. Neutral current neutrino interactions, caused by the Z boson, were observed in the Gargamelle experiment at CERN in the early 1970s and confirmed at Fermilab [2,11,33].

Finally, in 2000, the Direct Observation of the Nu Tau (DONUT) [34] experiment at Fermilab detected the last neutrino, the tau, by observing tau leptons produced by a tau neutrino beam. Because of the tau lepton's very short lifetime, a very high resolution detector was necessary to observe the tau lepton's production and decay. The short lifetime also makes identifying tau neutrino events difficult, and DONUT observed only five tau neutrino events, but this number was sufficiently larger than background to claim discovery.

The only experimental indication of beyond the Standard Model (BSM) physics is the existence of neutrino mass, made through discovery of neutrino oscillations. In the Standard Model, neutrinos are considered massless, and the flavor of a neutrino should not change as it travels through space. If that is the case, then electron neutrinos produced by the sun should be detected as electron neutrinos on the earth. In 1968, Ray Davis attempted to do this using a large tank of cleaning fluid. He hoped to detect solar neutrinos using the reaction $\nu_{e}+{ }^{37} \mathrm{Cl} \rightarrow{ }^{37} \mathrm{Ar}+e$ and looking for argon atoms in the tank. He detected just a third of the expected number of argon atoms. 
This deficit came to be known as the "solar neutrino problem." In the 1980s, groups studying atmospheric neutrinos noticed the ratio of $\nu_{\mu}$ to $\nu_{e}$ detected was around $1: 1$, whereas the expected ratio is closer to $2: 1[2,33]$.

While some claimed the deficit was due to an inaccurate model of the sun or experimental error, Pontecorvo proposed [35] that one flavor of neutirno could change into another, which Davis could not detect, a process called "oscillation." Solar neutrino oscillations were confirmed by the Sudbury Neutrino Observatory (SNO) in 2002 [36]. Unlike Davis' experiment, SNO is sensitive to all three types of neutrinos. Furthermore, because SNO used heavy water $\left(\mathrm{D}_{2} \mathrm{O}\right)$, it was able to separately identify interactions from just electron neutrinos and those from all neutrinos. This allowed SNO to determine the electron neutrino and total neutrino fluxes $[2,33]$.

Super-Kamiokande (Super-K) studied atmospheric neutrinos [37], and there too it found evidence of oscillations. Atmospheric neutrinos arise from decays of muons and charged pions produced by collisions of protons in the atmosphere. Super$\mathrm{K}$ examined the flux of neutrinos at various angles and found that those coming from directly overhead fit the expected flavor ratio of $2: 1$, but neutrinos coming from the other side of the earth did not. Those from the other side of the earth traveled a greater distance, so more oscillated into another flavor (tau neutrinos). Since atmospheric neutrinos are mostly muon flavor, this observation of oscillations is independent of that of solar neutrinos. Other experiments, using neutrinos from a variety of sources have provided further proof of these oscillations. In the 2000s, reactor and accelerator-based experiments reported their results of oscillations. K2K [38] and MINOS [39] studied $\nu_{\mu}$ disappearance. OPERA [40] studied $\nu_{\mu} \rightarrow \nu_{\tau}$ oscillation, while T2K [41] examined $\nu_{\mu} \rightarrow \nu_{e}$ oscillation, and in 2012, Daya Bay and RENO reported a precise measurement of $\theta_{13}$. These experiments have studied oscillations at different values of $L / E$, providing us with the mass differences of the three SM neutrinos as 
well as the mass range of a possible fourth neutrino. It is also worth mentioning that around this time, other experiments, such as LSND and MiniBooNE noticed anomalies suggesting a fourth neutrino, hinting at physics even further beyond the Standard Model [2, 28, 33].

\subsection{Sources of Neutrinos}

Neutrinos are produced from a variety of sources with a variety of energies. In addition, different sources produce different flavors of neutrinos. Figure 2.2 shows the sources and respective energies.

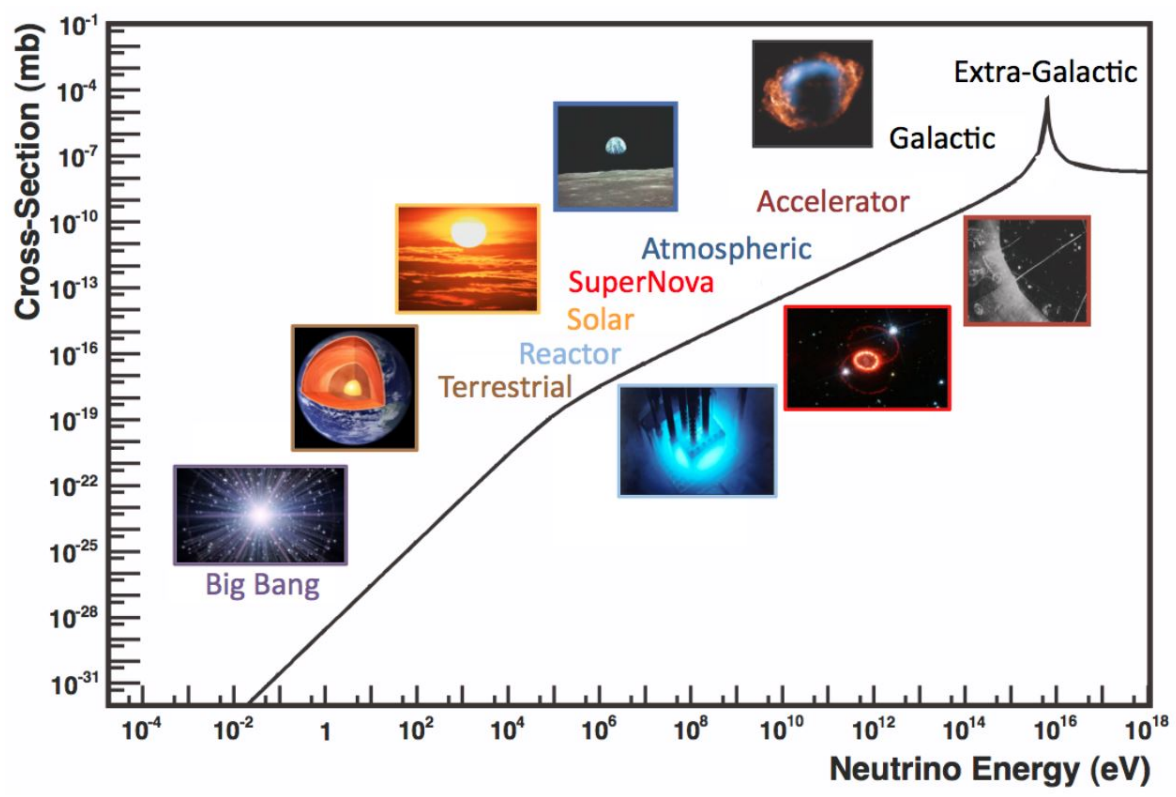

Figure 2.2. The sources and energies of neutrinos. The x-axis indicates the energy of the neutrino. The y-axis indicates the cross section of $\bar{\nu}_{e^{-}} e$ scattering. Image from $[5]$.

Reactors produce only electron antineutrinos, and reactor neutrino experiments seek to study neutrino oscillation and make measurements of reactor power, flux and fuel composition. The sun produces only electron neutrinos, while supernovae produce all flavors of neutrinos and antineutrinos. Experiments examining solar and supernova neutrinos seek to understand the processes stars undergo in the 
middle and end of their lives. Beams of mostly muon neutrinos and antineutrinos from accelerators are used to study oscillation and neutrino-matter interaction cross sections. Beam experiments have discovered two of the three known neutrino flavors and are currently searching for evidence of more (BSM) neutrino types. Finally, astrophysicists examine neutrinos from outer space. This thesis will focus on beam, solar and supernova neutrinos.

2.3.1 Beam Neutrinos. Particle accelerators produce neutrino beams by striking protons on a stationary target. The resulting interactions produce mesons, primarily pions and kaons. The charged mesons are then focused by magnetic horns; an illustration of the effect of the horns is found in Figure 2.3. The horns also filter mesons

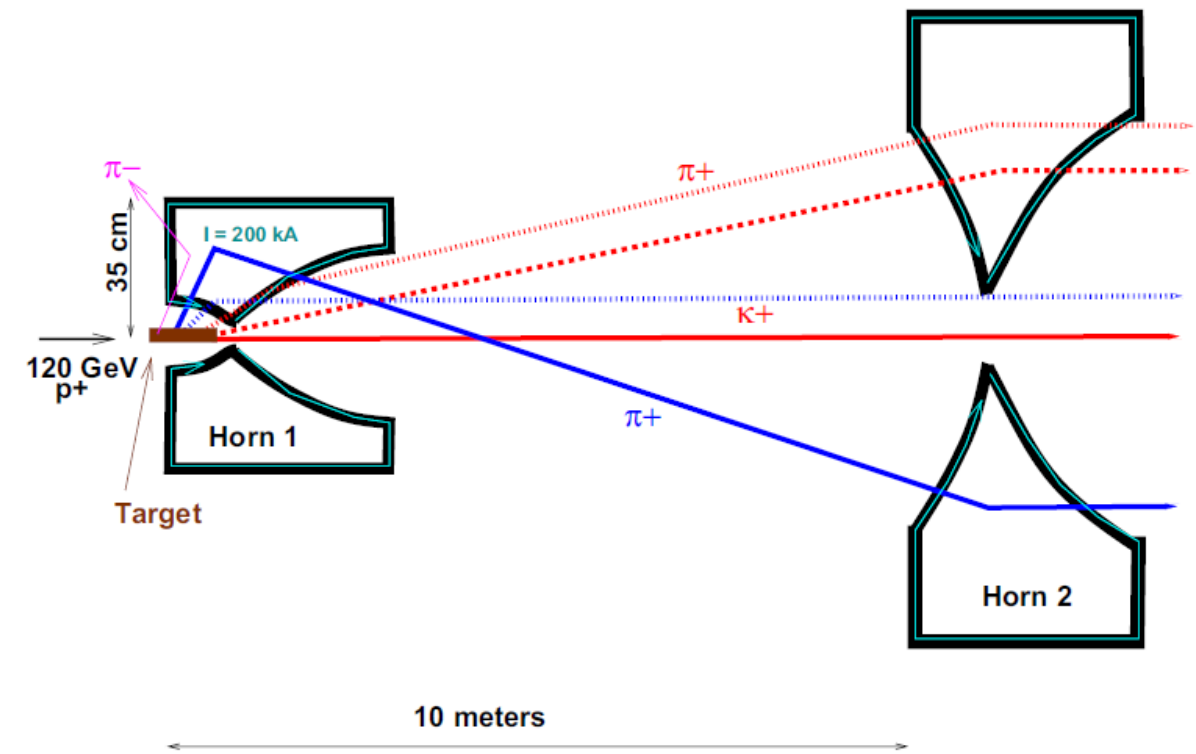

Figure 2.3. An illustration of the effect on the paths of charged hadrons from magnetic focusing horns. The proton beam enters from the left, striking the target and producing hadrons. The vertical scale is four times the horizontal scale. Image from $[6]$.

based on charge sign by changing the polarity of the horns. This in turn changes the ratio of neutrinos to antineutrinos. By adjusting the spacing of the horns, it is possible to produce higher or lower energy neutrino beams. After the horns, the mesons 
decay in the decay pipe, producing muons, muon neutrinos and antineutrinos and a small amount of electron neutrinos and antineutrinos, as shown in Eqs. 2.6 - 2.9. These neutrinos then pass through rock, which stops all particles except neutrinos. Unlike charged particle beams, the neutrinos in a beam do not all have the same energy, and the beam itself covers a degrees-wide angular distribution.

$$
\begin{aligned}
\pi^{-} \rightarrow \mu^{-}+\bar{\nu}_{\mu} & \pi^{+} \rightarrow \mu^{+}+\nu_{\mu} \\
K^{+} \rightarrow \mu^{+}+\nu_{\mu} & K^{-} \rightarrow \mu^{-}+\bar{\nu}_{\mu} \\
\mu^{-} \rightarrow e^{-}+\bar{\nu}_{e}+\nu_{\mu} & \mu^{+} \rightarrow e^{+}+\nu_{e}+\bar{\nu}_{\mu} \\
K^{+} \rightarrow \pi^{0}+e^{+}+\nu_{e} & K^{-} \rightarrow \pi^{0}+e^{-}+\bar{\nu}_{e}
\end{aligned}
$$

There are two neutrino beams currently used at Fermilab: the Neutrinos at the Main Injector (NuMI) beam and the Booster Neutrino Beam (BNB). The NuMI beam is of higher energy and is used for long-baseline experiments. The BNB is used for short-baseline experiments. The neutrino beams are created by protons sent through the Fermilab accelerator complex, which begins with a bottle of hydrogen. An extra electron is added to the hydrogen atom, giving it a negative charge. These ions are accelerated in the Pre-Accelerator to $750 \mathrm{keV}$. The ions are then sent into the Linear Accelerator which accelerates them to $400 \mathrm{MeV}$. Both electrons are then stripped off, and the protons enter a synchrotron called the Booster which accelerates them to $8 \mathrm{GeV}$. After the Booster, some protons are directed to the BNB target, while others are sent to the Main Injector. The Main Injector accelerates protons to 120 $\mathrm{GeV}$ after which they are sent to the NuMI target or other fixed target experiments.

The NuMI beam was designed for the Main Injector Neutrino Oscillation Search (MINOS) experiment [42] and is the beam used for three other Fermilab experiments, NO $\nu \mathrm{A}, \mathrm{MINER} \nu \mathrm{A}$ and ArgoNeuT. The NuMI beam uses $120 \mathrm{GeV}$ pro- 
tons from the Main Injector which strike a graphite target. The target is $15 \mathrm{~mm}$ tall, $6.4 \mathrm{~mm}$ wide and $95.38 \mathrm{~cm}$ long. The infrastructure also features two magnetic horns, a hadron monitor, hadron absorber and three muon monitors, shown in Figure 2.4.

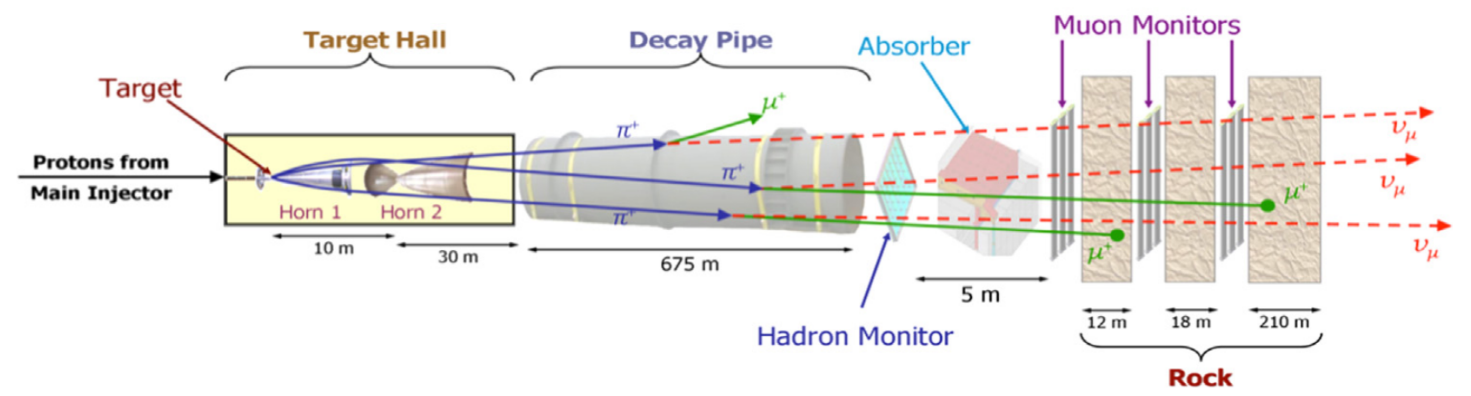

Figure 2.4. Diagram of infrastructure for the NuMI beam. Image from [6].

The NuMI beam can be operated in several modes. By adjusting the polarity of the horns, either positively or negatively charged hadrons can be focused. Thus, the beam can be operated in neutrino mode, where the beam is mostly (94\%) muon neutrinos, or in antineutrino mode, where the beam is $58 \%$ muon neutrino and $40 \%$ muon antineutrino. The beam also contains also a small amount of electron neutrinos and antineutrinos. When the NuMI beam was designed, the neutrino mass difference $\Delta m_{32}^{2}$, an important number for oscillation studies, was not well known. Since neutrino oscillation probabilities depend on this value, the MINOS collaboration wanted a beam whose energy could be modified depending on the value of $\Delta m_{32}^{2}$. Consequently, one of the horns can be moved, allowing for changes to the energy spectrum of the beam. The NuMI beam has three configurations: low, medium and high-energy. The spectra of these configurations is given in Figure 2.5. During the majority of the beam's operation, the low-energy setting was used. The profile of this mode is seen in Figure 2.6; more detail about the construction and operation of the NuMI beam is given in [6].

To create the $\mathrm{BNB}, 8 \mathrm{GeV}$ protons from the Booster strike a Beryllium target. 


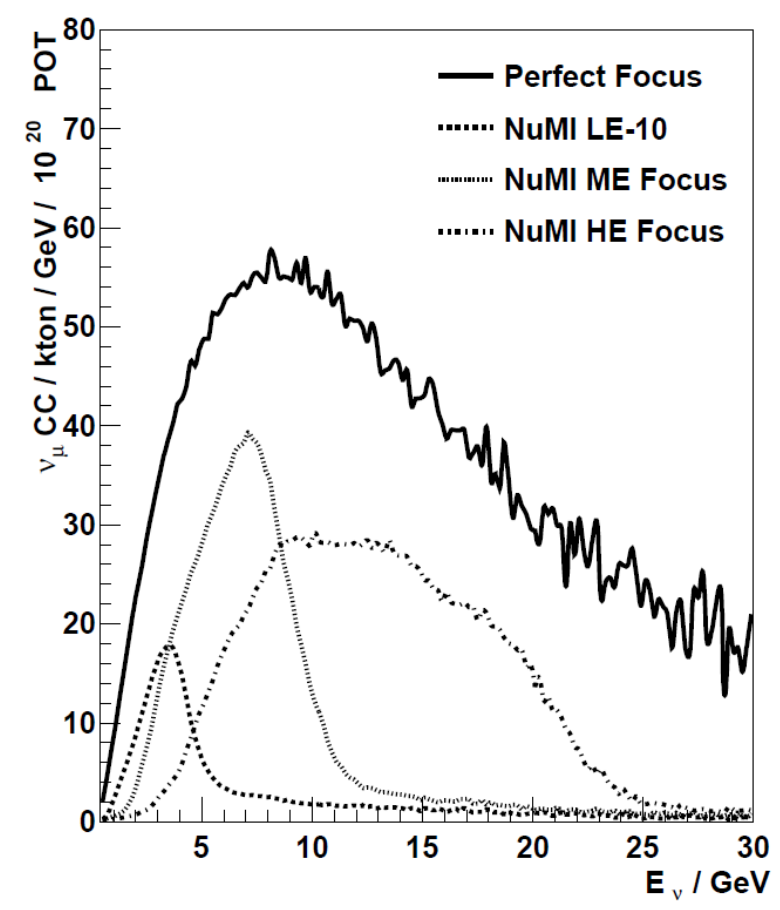

Figure 2.5. Flux of neutrinos from the NuMI beam with different horn settings. Perfect focusing refers to every pion being perfectly focused along the beam axis. The $y$-axis indicates the event rate at the MINOS far detector; it is included here to describe the relative fluxes. Image from [6].

There is only one fixed horn, so while the polarity of the horn can be changed, the energy spectrum cannot. The BNB does not have any hadron monitors, but does have a muon counter located at an angle off the beamline. Because the proton beam is of lower energy, the neutrino spectrum, shown in Figure 2.7, is also of lower energy.

Beam neutrinos have several advantages over other sources. For one, the source can be turned on or off, allowing an experiment to obtain data describing non-beam backgrounds. Backgrounds can be minimized by reading out the detector only when neutrinos are expected to arrive at the detector. This is known due to the timing of the accelerator. The energy and flavor spectrum of the neutrinos can also be changed by changing the polarity of the horns and/or by changing the spacing of the horns. While accelerators produce mostly muon neutrinos and antineutrinos, with some electron 


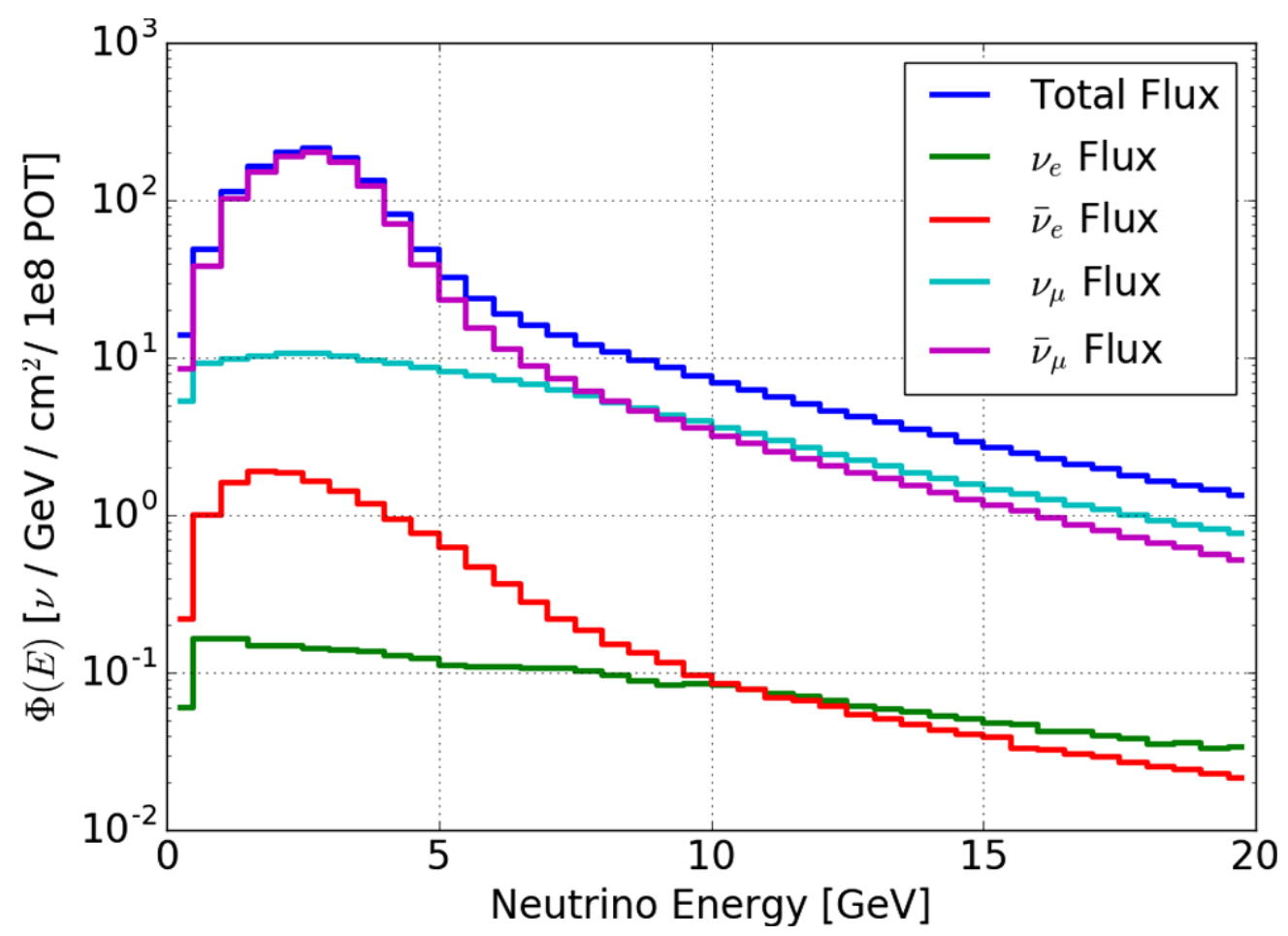

Figure 2.6. Flux of neutrinos from the NuMI beam operating in the low-energy, antineutrino mode and entering ArgoNeuT, a liquid argon detector, $1 \mathrm{~km}$ downstream. Image from [7].

(anti)neutrinos, it is also possible to produce tau neutrinos, as was done for the DONUT experiment. Furthermore, as shown in Figure 2.2, higher energy neutrinos have larger cross-sections, which results in more detections. Accelerator sources do have some disadvantages. For one, an accelerator is expensive to operate. In addition, the beam is not perfectly flavor/matter pure, unlike the sun and nuclear reactors.

2.3.2 Solar Neutrinos. The sun is powered by nuclear fusion, which also makes it a source of neutrinos. For all main sequence stars, the primary process is the proton-proton (pp) chain, which begins with the following reaction:

$$
p+p \rightarrow d+e^{+}+\nu_{e}+0.42 \mathrm{MeV} .
$$

It is also possible, but far less common ( $0.4 \%$ probability), to produce deuterium $d$ by the reaction

$$
p+e^{-}+p \rightarrow d+\nu_{e}+1.442 \mathrm{MeV},
$$




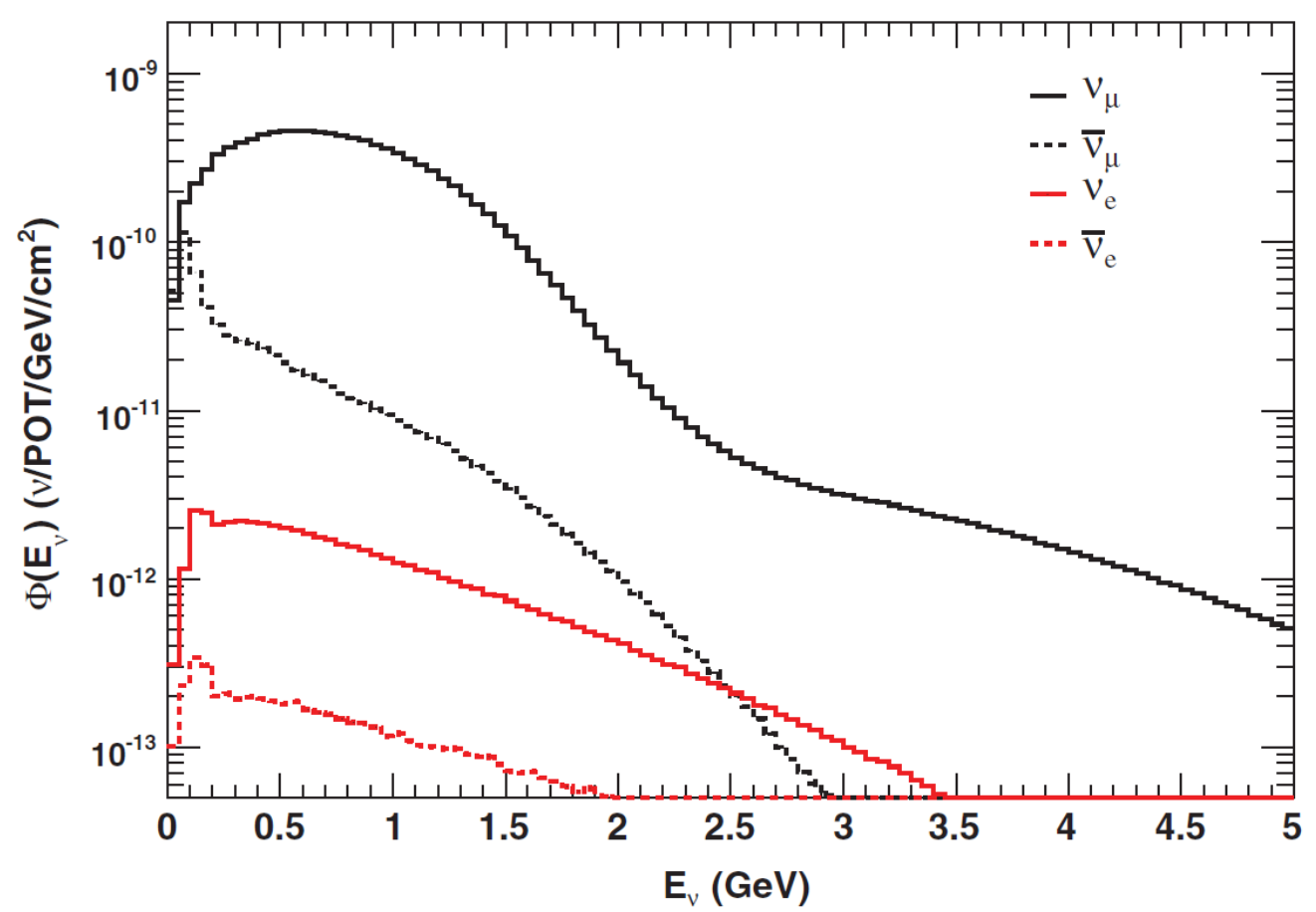

Figure 2.7. Flux of neutrinos from the BNB beam at MiniBooNE. The flux is roughly the same for all BNB experiments. Image from [8].

called the "pep" reaction. The neutrinos in this reaction are monoenergetic at 1.442 $\mathrm{MeV}$. The deuterium can then react with another proton to produce ${ }^{3} \mathrm{He}$ and a photon. There are many possible reactions involving ${ }^{3} \mathrm{He}$. One is called the "hep" reaction:

$$
{ }^{3} \mathrm{He}+p \rightarrow{ }^{4} \mathrm{He}+e^{+}+\nu_{e}+18.77 \mathrm{MeV}
$$

Alternatively, the ${ }^{3} \mathrm{He}$ can then react with another ${ }^{3} \mathrm{He}$ to produce ${ }^{4} \mathrm{He}$, or it can react with a ${ }^{4} \mathrm{He}$ to produce ${ }^{7} \mathrm{Be}$ and a photon. The ${ }^{7} \mathrm{Be}$ can interact with an electron,

$$
{ }^{7} \mathrm{Be}+e^{-} \rightarrow{ }^{7} \mathrm{Li}+\nu_{e}+0.862 \mathrm{MeV}
$$

producing ${ }^{7} \mathrm{Be}$ neutrinos, which are monoenergetic at $0.862 \mathrm{MeV}$. There is a small probability $(0.02 \%)$ that ${ }^{7} \mathrm{Be}$ will react with a proton, producing ${ }^{8} \mathrm{~B}$. The ${ }^{8} \mathrm{~B}$ decays, producing ${ }^{8} \mathrm{~B}$ neutrinos:

$$
{ }^{8} \mathrm{~B} \rightarrow{ }^{8} \mathrm{Be}^{*}+e^{+}+\nu_{e}+15.04 \mathrm{MeV}
$$


For stars with masses around one solar mass and above, the pp chain is not enough. The next step is the Carbon-Nitrogen-Oxygen (CNO) cycle, in which protons fuse with carbon and nitrogen, producing nitrogen and oxygen, respectively. The nitrogen and oxygen undergo beta decay, producing more electron neutrinos. The fluxes of these sources are given in Figure 2.8. More information about these processes can be found in $[9,43,44]$.

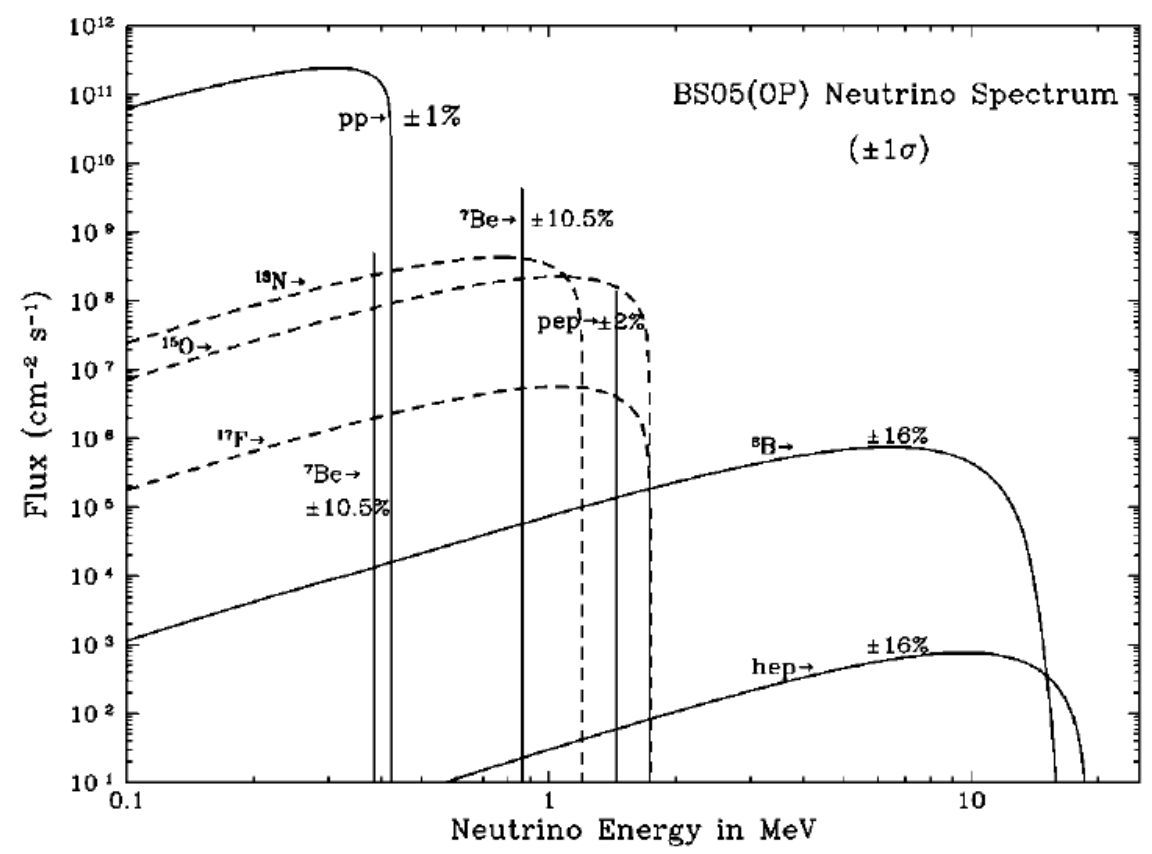

Figure 2.8. Solar neutrino flux spectrum listing the sources mentioned in the text. Image from [9].

Solar neutrino experiments have provided us with oscillation measurements [45] as well as a measurement of the MSW effect on oscillations in matter [46]. These measurements have also validated the Standard Solar Model by measuring the predicted neutrino flux. Future measurements are described in detail in $[3,47]$.

2.3.3 Supernova Neutrinos. Supernovae are one of the most spectacular events in the universe. They arise at the end of the life of a star, when the star explodes. A supernova can come about in two possible ways. In one, a white dwarf gains mass 
by accretion from a companion star. In the other, the iron core of a massive star collapses. Studies of neutrinos from supernovae can provide information on not just supernovae but also on neutrinos themselves. Supernova neutrinos are low energy (tens of MeV), compared to beam neutrinos $[11,27,48]$.

A Type II supernova occurs at the end of all stages of nuclear burning. Stars more massive than the sun undergo stages of fusion beyond those already described, and stars with a mass greater than ten solar masses undergo all stages, ending with silicon burning. Silicon burning produces iron, which does not undergo fusion. The star is then left with an iron core. Surrounding the iron core are shells of silicon, oxygen, neon, carbon, helium and hydrogen. Until this point, fusion has kept the star's core from collapsing in on itself. After this, degenerate electrons will prevent the collapse, but as the shell grows, the electrons become relativistic. The electrons will fail to prevent the core from collapsing once the the mass of the core reaches the critical point of the Chandrasekhar mass. Furthermore, the high temperature and density of the core allow for two processes which absorb energy from the core and trigger the collapse of the core, nuclear photodisintegration and electron capture. In nuclear photodisintegration, thermal photons break iron nuclei into nuclei that are not as tightly bound. The amount of energy lost by the core is enormous, equal to the amount of energy the sun radiates in 10 billion years. In electron capture, an electron is captured by a proton to produce a neutron and electron neutrino. Since neutrons are formed, this process is called "neutronization." When the density of the iron core is high enough, the Fermi energy of the electrons is sufficiently high to overcome the energy barrier for electron capture. The density of electrons in the core is so high that beta decay cannot occur, and the electron capture process continues. The amount of energy lost via this process is of the same order as that via photodisintegration; in this case, the energy is lost via electron neutrinos. The core stops collapsing when the density of the core is greater than nuclear matter. This is followed by a shock 
wave which produces an explosion. Following the explosion, a neutron star is left. The last two of these stages are extremely complex and only partially understood; see Ref. [49] for a brief overview. If this neutron star is massive enough, a black hole will form $[11,27,48]$.

The production of supernova neutrinos during the core-collapse of a massive star can be divided into three stages. The first step is neutronization which produces $\nu_{e}$ from electron capture and lasts tens of milliseconds. Next comes the accretion phase, lasting tens to hundreds of milliseconds, where again $\nu_{e}$ are produced. The final phase is the cooling phase. This segment, which lasts tens of seconds causes most of the gravitational binding energy to be lost. Neutrino-antineutrino pairs of all flavors are produced in almost equal amounts. This "neutrino blackbody cooling" is the primary method of energy release and dictates the energy spectrum of the neutrinos. Electron neutrinos interact most with the matter inside the core because of the large number of neutrons present. Electron antineutrinos can interact with the protons left in the core, so the number of their interactions is larger than for all remaining neutrino flavors $(\mu, \tau$, and their antimatter counterparts, collectively called $\left.\nu_{x}\right)$. These $\nu_{x}$ are not energetic enough to create their corresponding charged lepton, so they can only undergo neutral current interactions. Neutral current interactions take away less energy from the neutrino, so there is a hierarchy of the average energy of neutrino flavors: $\left\langle E_{\nu_{x}}\right\rangle>\left\langle E_{\bar{\nu}_{e}}\right\rangle>\left\langle E_{\nu_{e}}\right\rangle$. The spectrum of neutrinos produced is given in Figure 2.9. It is important to note that oscillation effects in such a dense medium are not fully understood and could substantially alter the spectrum seen in the Figure $[11,27,48]$.

The only core collapse supernova observed by neutrino detectors was supernova 1987A, detected on February 23, 1987. Three detectors, Kamiokande, IMB and Baksan, saw a total of 24 events over 13 seconds caused by interactions of neutrinos 


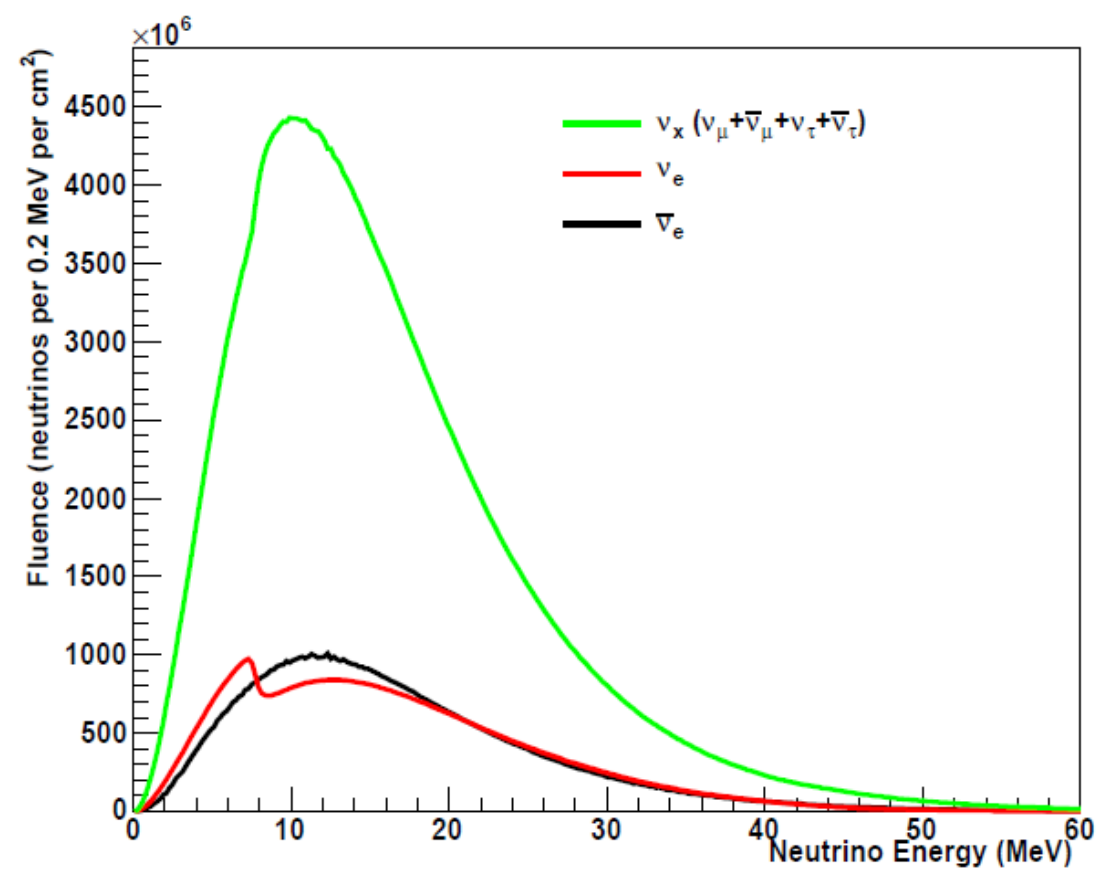

Figure 2.9. Supernova neutrino spectrum. The dip in the $\nu_{e}$ spectrum is due to collective effects. Note that oscillation effects in such a dense medium are not fully understood and could substantially alter the spectrum. Image from [10].

from the supernova. The relative event times and energies of the neutrinos are given in Figure 2.10. All of the neutrinos detected were $\bar{\nu}_{e}$ with an average energy of 12.5 $\mathrm{MeV}$. Calculating the flux using these observed neutrinos reveals that around $10^{53} \mathrm{erg}$ was released from the supernova; this number agrees with supernova models. Since the flux matched expectations, a lower limit on the lifetime of the neutrino can be set: $10^{5}$ years. In addition, from the spread of arrival times, limits on the mass of the neutrino can be placed: $m_{\bar{\nu}_{e}}<5.70 \mathrm{eV}[11]$.

While photons may take several hours to exit a supernova due to interactions, neutrinos escape more quickly. Because of this, they provide a better picture of the activity inside a supernova, such as the processes occuring at each phase. The neutrino signal will test the accuracy of models of supernova neutrino production, such as the time and flavor profiles. In addition to learning about supernovae, supernova 


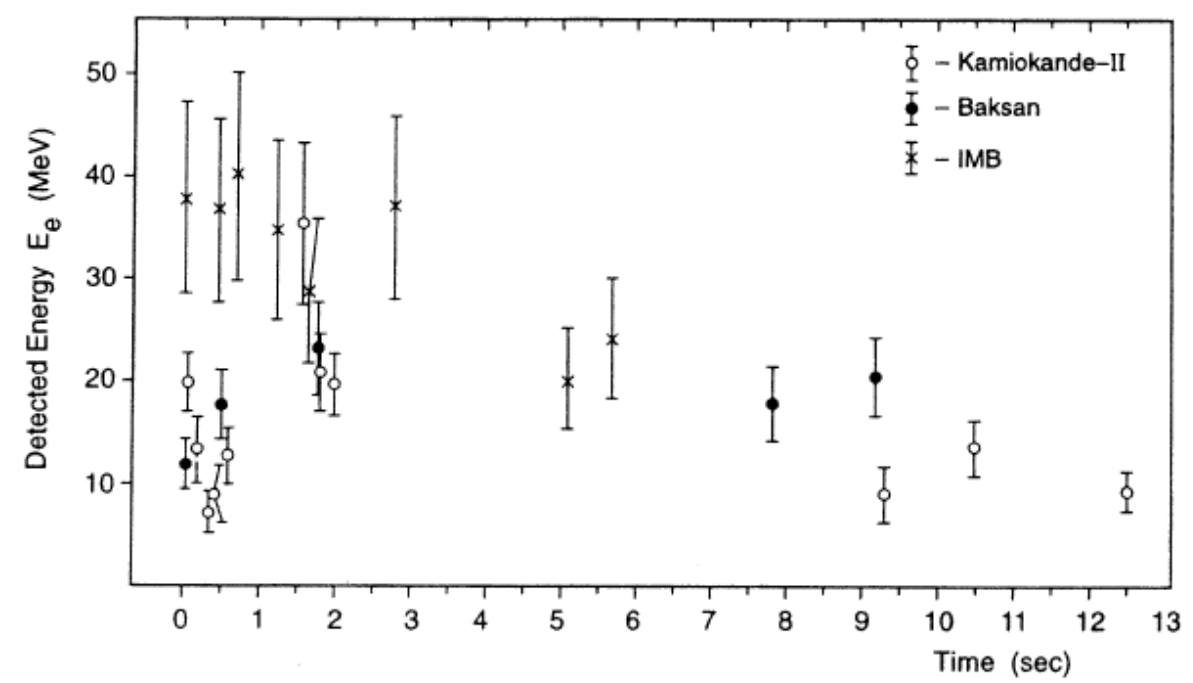

Figure 2.10. Relative event times and energies of neutrinos from SN1987A. Image from [11].

neutrinos provide insight into neutrinos themselves. Studies of supernova neutrino flavors and energies provide another probe of the mass hierarchy and one of the mixing angles. This is possible because both neutrinos and antineutrinos are produced, but the spectra of $\nu_{e}$ and $\bar{\nu}_{e}$ depends on the mass hierarchy. Finally, they can also provide an early warning for astronomers wishing to optically observe a supernova. By detecting elastic scattering of neutrinos with electrons, it is possible to determine the location of the supernova using the direction of the scattered electron [10,27,50-53].

\subsection{Neutrino Interactions}

Neutrinos only interact with matter via the weak force, mediated by either the neutral $\mathrm{Z}$ boson or the charged $\mathrm{W}$ boson. In the former case, the interaction is a neutral current (NC) interaction, while in the latter it is a charged current (CC) interaction. Charged current interactions result in the production of a charged lepton, the flavor of which depends on the flavor of neutrino that interacted. For this to occur, the energy of the neutrino must be higher than the mass of the charged lepton. A $\mathrm{NC}$ interaction produces no charged lepton. Experimentally, CC interactions can 
be used to study oscillations between the three active neutrinos, as they produce a lepton which identifies the flavor of the interacting neutrino. On the other hand, $\mathrm{NC}$ interactions produce no lepton, so it is impossible to determine the flavor of the interacting neutrino.

At neutrino energies of around $0.1-20 \mathrm{GeV}$, common for accelerator produced neutrinos, interactions are typically further classified as one of four types, for both $\mathrm{CC}$ and NC interactions. The first is quasi-elastic scattering, shown in Figure 2.11, where the neutrino elastically scatters off a nucleon. In CC neutrino interactions, a proton is turned into a neutron, while in CC antineutrino interactions, a neutron turns into a proton. This interaction for both neutrinos and antineutrinos is shown in Eqs. $2.15-2.17$ below.
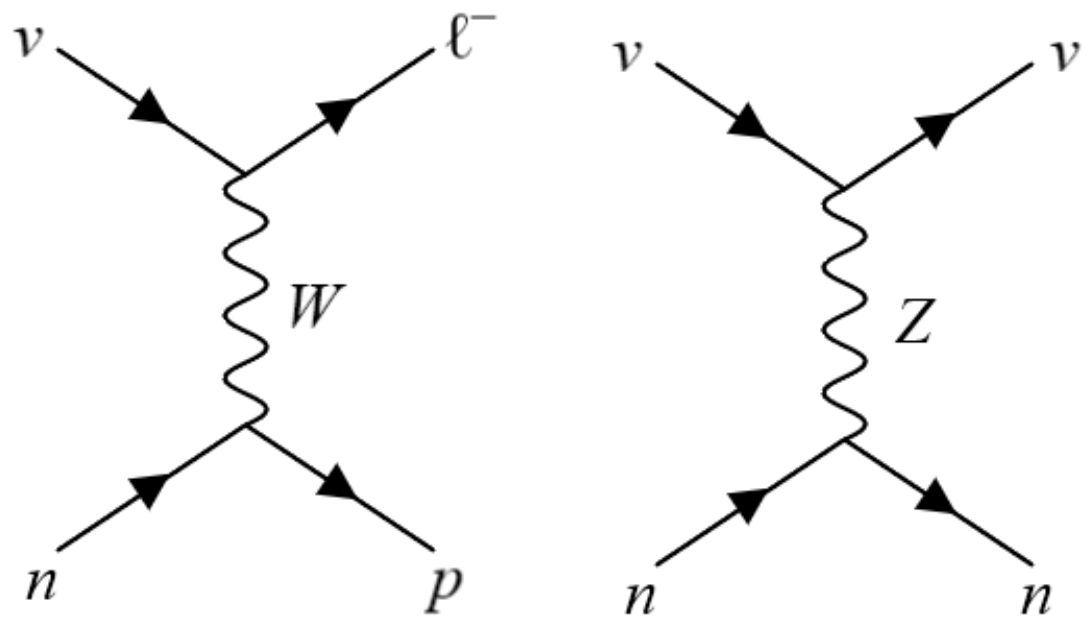

Figure 2.11. Charged current (left) and neutral current (right) neutrino interactions on neutrons. Note that the neutral current reaction produces no charged lepton.

$$
\begin{array}{cc}
\nu_{e}+n \rightarrow e^{-}+p & \bar{\nu}_{e}+p \rightarrow e^{+}+n \\
\nu_{\mu}+n \rightarrow \mu^{-}+p & \bar{\nu}_{\mu}+p \rightarrow \mu^{+}+n \\
\nu_{\tau}+n \rightarrow \tau^{-}+p & \bar{\nu}_{\tau}+p \rightarrow \tau^{+}+n
\end{array}
$$


In both $\mathrm{CC}$ and $\mathrm{NC}$ interactions, the nucleon is freed. As the freed nucleon travels within the nucleus, it can scatter off of other nucleons, freeing them as well. This is referred to as "final-state interactions." A CCQE interaction could thus have multiple protons and neutrons emitted. The nucleus itself can be left in an excited state. To de-excite, the nucleus will release photons and possibly other nucleons. A study of these de-excitation photons will be discussed later.

The second class of interactions is resonance production which is an inelastic interaction. Here the struck nucleon is excited, creating a baryon resonance. When the resonant state decays, nucleons and mesons are produced, commonly pions, as shown in Eqs. 2.18 - 2.20 for CC interactions and Eqs. 2.21 - 2.24 for NC interactions, where $l$ can be $e, \mu$ or $\tau$.

$$
\begin{array}{cc}
\nu_{l}+p \rightarrow l^{-}+p+\pi^{+} & \bar{\nu}_{l}+p \rightarrow l^{+}+p+\pi^{-} \\
\nu_{l}+n \rightarrow l^{-}+p+\pi^{0} & \bar{\nu}_{l}+p \rightarrow l^{+}+p+\pi^{0} \\
\nu_{l}+n \rightarrow l^{-}+n+\pi^{+} & \bar{\nu}_{l}+n \rightarrow l^{+}+n+\pi^{-} \\
& \\
\nu_{l}+p \rightarrow \nu_{l}+p+\pi^{0} & \bar{\nu}_{l}+p \rightarrow \bar{\nu}_{l}+p+\pi^{0} \\
\nu_{l}+p \rightarrow \nu_{l}+n+\pi^{+} & \bar{\nu}_{l}+n \rightarrow \bar{\nu}_{l}+n+\pi^{0} \\
\nu_{l}+n \rightarrow \nu_{l}+n+\pi^{0} & \bar{\nu}_{l}+n \rightarrow \bar{\nu}_{l}+n+\pi^{0} \\
\nu_{l}+n \rightarrow \nu_{l}+p+\pi^{-} & \bar{\nu}_{l}+n \rightarrow \bar{\nu}_{l}+p+\pi^{-}
\end{array}
$$

The baryon resonance can also decay to one or more photons. While the branching ratio of the latter decay is small, it does provide a background to some oscillation studies, as e.g. Cherenkov detectors cannot distinguish between an electron and a photon. Single pions may also be produced by coherent scattering. In coherent scattering, a neutrino scatters with the nucleus $(N)$ providing little energy to the 
nucleus. The result is a forward-going pion and a nucleus left in its ground state. The $\mathrm{CC}$ and $\mathrm{NC}$ versions of this interaction are given in Eqs. 2.25 and 2.26, respectively.

$$
\begin{array}{cc}
\nu_{l}+N \rightarrow \mu^{-}+N+\pi^{+} & \bar{\nu}_{l}+N \rightarrow \mu^{+}+N+\pi^{-} \\
\nu_{l}+N \rightarrow \nu_{l}+N+\pi^{0} & \bar{\nu}_{l}+N \rightarrow \bar{\nu}_{l}+N+\pi^{0}
\end{array}
$$

The final interaction type is deep inelastic scattering, where the neutrino interacts with a quark in the nucleon. This type of interaction is typically seen in experiments as a shower of hadrons, since the scattered quark combines with other quark(s). As shown in Figure 2.12, quasi-elastic interactions dominate at lower energies, while deep inelastic scattering dominates at higher energies [5].

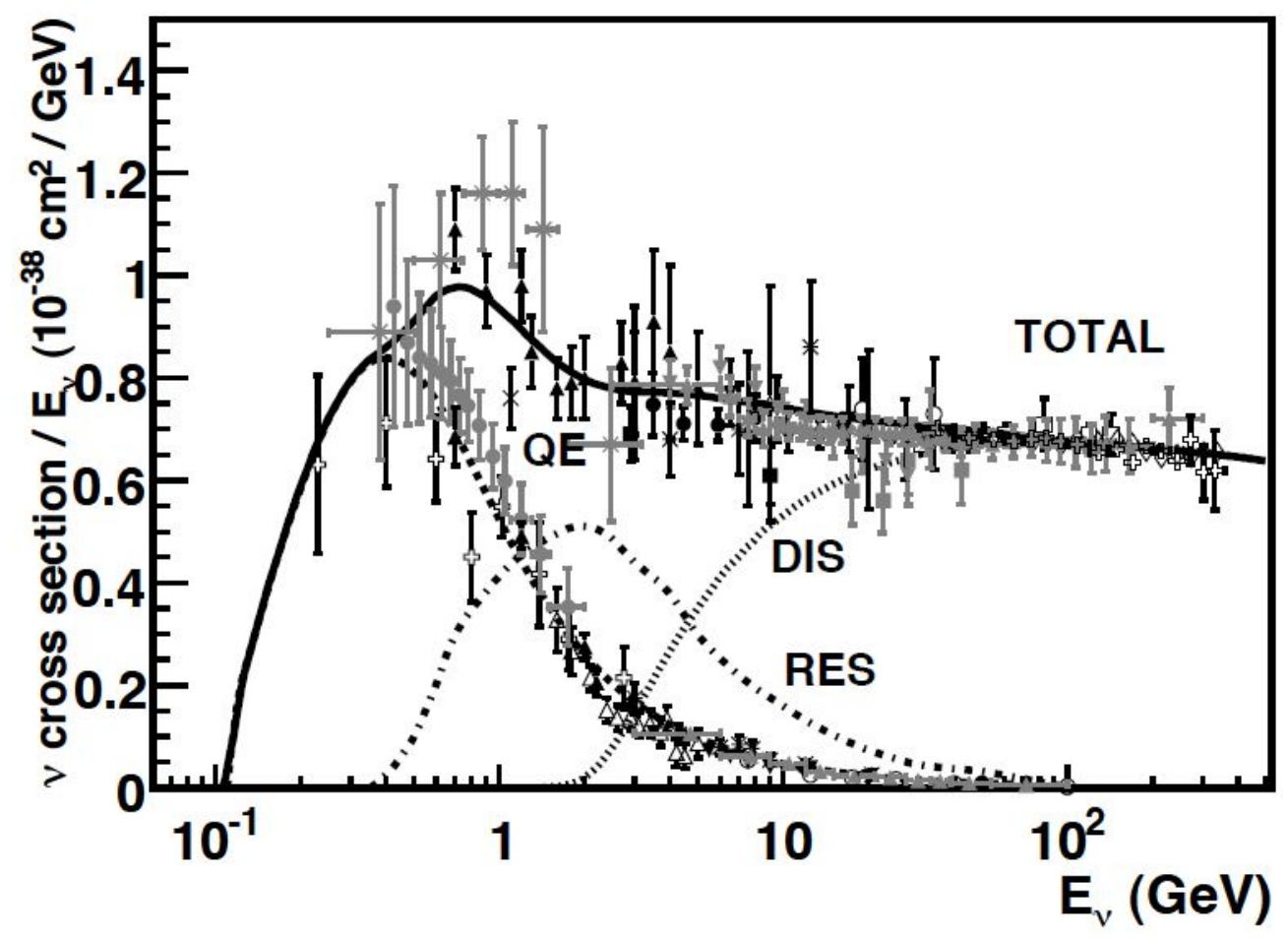

Figure 2.12. Measurements of cross sections for quasielastic scattering, resonance production and deep inelastic scattering for neutrinos. From [5].

It must be noted that experiments typically classify interactions based on their topology, i.e. the particles detected. Experiments are unable to see what occurs in 
the nucleus (the final state interactions); charged particles have energy thresholds for detection, and neutral particles are difficult to see, so it is difficult to determine exactly the category of interaction (quasi-elastic, resonant production or DIS) that occurred. With a high-resolution detector with low thresholds, one can see more of the activity exiting the final state nucleus. LArTPCs have high resolution (few millimeter) and low thresholds (10 MeV for pions, $21 \mathrm{MeV}$ for protons) and are thus able to observe complex topologies, for example protons emitted back-to-back [17] (also Fig. 4.2).

\subsection{Neutrino Oscillations}

As mentioned earlier, the only confirmed physics beyond the Standard Model is neutrino oscillation. This is due to the fact that neutrinos have mass, and that their mass eigenstates are not the same as their flavor eigenstates. This section will explore the theory behind neutrino oscillations, tests of this theory, consequences of oscillations and planned studies.

We will begin by deriving the equations for neutrino oscillation. This follows from [29]. A neutrino in a flavor state $\left|\nu_{\alpha}\right\rangle(\alpha=e, \mu, \tau)$ can be described as a superposition of massive neutrino states $\left|\nu_{i}\right\rangle$ :

$$
\left|\nu_{\alpha}\right\rangle=\sum_{i} U_{\alpha i}^{*}\left|\nu_{i}\right\rangle
$$

where the mixing matrix $U_{\alpha i}^{*}$ is the weight of the mass state in the flavor state. The massive neutrino states are eigenstates of the Hamiltonian, and their time evolution can be described as

$$
\left|\nu_{i}(t)\right\rangle=e^{-i E_{i} t}\left|\nu_{i}\right\rangle
$$

where $E_{i}=\sqrt{p^{2}+m_{i}^{2}}$ is the energy eigenvalue. This implies the flavor state's $\left(\left|\nu_{\alpha}\right\rangle\right)$ evolution over time is given by

$$
\left|\nu_{\alpha}(t)\right\rangle=\sum_{i} U_{\alpha i}^{*} e^{-i E_{i} t}\left|\nu_{i}\right\rangle
$$


Now, since $U$ is unitary, the mass states can be written in terms of the flavor states:

$$
\left|\nu_{i}\right\rangle=\sum_{\alpha} U_{\alpha i}\left|\nu_{\alpha}\right\rangle
$$

If we insert Eq. 2.30 into Eq. 2.29, we obtain the time evolution of a flavor state in terms of flavor states:

$$
\left|\nu_{\alpha}(t)\right\rangle=\sum_{\beta}\left(\sum_{i} U_{\alpha i}^{*} U_{\beta i} e^{-i E_{i} t}\right)\left|\nu_{\beta}\right\rangle,
$$

where $\beta=e, \mu, \tau$. The probability of oscillating from one flavor to another as a function of time can be obtained by taking the modulus squared of Eq. 2.31.

$$
P_{\nu_{\alpha} \rightarrow \nu_{\beta}}(t)=\left|\left\langle\nu_{\beta} \mid \nu_{\alpha}(t)\right\rangle\right|^{2}=\sum_{i, j} U_{\alpha i}^{*} U_{\beta i} U_{\alpha j} U_{\beta j}^{*} e^{i\left(E_{i}-E_{j}\right) t} .
$$

For ultrarelativistic neutrinos,

$$
E_{i} \simeq E+\frac{m_{i}^{2}}{2 E}
$$

so

$$
E_{i}-E_{j} \simeq \frac{m_{i}^{2}-m_{j}^{2}}{2 E}
$$

Defining $\Delta m_{i j}^{2} \equiv m_{i}^{2}-m_{j}^{2}$, Eq. 2.32 can be written as

$$
P_{\nu_{\alpha} \rightarrow \nu_{\beta}}(t)=\sum_{i, j} U_{\alpha i}^{*} U_{\beta i} U_{\alpha j} U_{\beta j}^{*} \exp \left[-i \frac{\Delta m_{i j}^{2} t}{2 E}\right] .
$$

Oscillation experiments know the distance $L$ traveled by the neutrino, but not the time. Assuming that the neutrino travels at (almost) the speed of light, so $t=L$, Eq. 2.35 can be written as

$$
P_{\nu_{\alpha} \rightarrow \nu_{\beta}}(t)=\sum_{i, j} U_{\alpha i}^{*} U_{\beta i} U_{\alpha j} U_{\beta j}^{*} \exp \left[-i \frac{\Delta m_{i j}^{2} L}{2 E}\right] .
$$

For antineutrinos, one takes the complex conjugate of the mixing matrices, thus

$$
P_{\bar{\nu}_{\alpha} \rightarrow \bar{\nu}_{\beta}}(t)=\sum_{i, j} U_{\alpha i} U_{\beta i}^{*} U_{\alpha j}^{*} U_{\beta j} \exp \left[-i \frac{\Delta m_{i j}^{2} L}{2 E}\right] .
$$


The theory of neutrino oscillations is considerably simplified if one examines just two flavors of neutrinos. If there are two flavors of neutrinos, $\nu_{e}$ and $\nu_{\mu}$, and if a neutrino oscillates between the two flavors, then neither flavor is a eigenstate of the Hamiltonian. We will call the true eigenstates $\nu_{1}$ and $\nu_{2}$. We can convert between the flavors and eigenstates in the following way:

$$
\left(\begin{array}{c}
\nu_{1} \\
\nu_{2}
\end{array}\right)=\left(\begin{array}{cc}
\cos \theta & -\sin \theta \\
\sin \theta & \cos \theta
\end{array}\right)\left(\begin{array}{l}
\nu_{e} \\
\nu_{\mu}
\end{array}\right) .
$$

If we apply the usual time dependence $e^{-i E_{i} t / \hbar}$ to $\nu_{1}$ and $\nu_{2}$ to the initial states, we obtain

$$
\nu_{1}(t)=-\sin \theta e^{-i E_{1} t / \hbar}, \nu_{2}(t)=\cos \theta e^{-i E_{2} t / \hbar} .
$$

If we feed this into the original matrix (Eq. 2.38), solve for $\nu_{\mu}$ and take the modulus squared, we obtain the probability of an electron neutrino turning into a muon neutrino. We can generalize this and write the probability of a neutrino of flavor $\alpha$ turning into the other flavor $\beta$, also called the "appearance probability" as:

$$
P_{\nu_{\alpha} \rightarrow \nu_{\beta}}=\sin ^{2} 2 \theta \sin ^{2}\left(\frac{\Delta m^{2} c^{3} L}{4 \hbar E}\right),
$$

where $\Delta m^{2}=m_{\beta}^{2}-m_{\alpha}^{2}$ is the difference of the squares two mass states, $L$ is the baseline, or the distance at which the neutrinos are detected, and $\mathrm{E}$ is the energy of the neutrino. Experiments measure the appearance and/or disappearance of neutrinos.

While the two-flavor approximation is quite useful in most experiments, it is worth including the matrix which governs three-flavor neutrino oscillation, called the Pontecorvo-Maki-Nakagawa-Sakata (PMNS) matrix:

$$
U_{\mathrm{PMNS}}=\left(\begin{array}{ccc}
c_{12} c_{13} & s_{12} c_{13} & s_{13} e^{-i \delta} \\
-s_{12} c_{23}-c_{12} s_{23} s_{13} e^{-i \delta} & c_{12} c_{23}-s_{12} s_{23} s_{13} e^{-i \delta} & s_{23} c_{13} \\
s_{12} s_{23}-c_{12} c_{23} s_{13} e^{-i \delta} & -c_{12} s_{23}-s_{12} c_{23} s_{13} e^{-i \delta} & c_{23} c_{13},
\end{array}\right)
$$


where $c_{i j}=\cos \theta_{i j}, s_{i j}=\sin \theta_{i j}, i, j \in\{1,2,3\}, i \neq j$ and $\delta$ is the CP-violating phase. If neutrinos are their own antiparticles, called Majorana fermions (Dirac fermions if not), then the matrix in Eq. 2.41 needs to be multiplied on the right by another, diagonal matrix:

$$
\left(\begin{array}{ccc}
1 & 0 & 0 \\
0 & e^{\frac{i}{2} \alpha_{21}} & 0 \\
0 & 0 & e^{\frac{i}{2} \alpha_{31}}
\end{array}\right)
$$

where $\alpha_{21}$ and $\alpha_{31}$ are the two Majorana CP violating phases.

Several experiments have studied neutrino oscillations. This is typically done with the use of two or more detectors, one placed closer to the source of neutrinos (called a "near detector") and one placed further from the source of neutrinos ("far detector"). The near detector is usually placed close to the source of neutrinos and is used to study the flux of neutrinos before oscillation occurs. The far detector is usually placed at a distance where neutrino oscillation is maximal (see Eq. 2.40). For reactor neutrino experiments, this distance can be as low as a few meters, while for accelerator neutrino experiments it may be hundreds of kilometers. Accelerator neutrino experiments such as MINOS, K2K, T2K and NO $\nu$ A have determined the atmospheric mixing angle $\theta_{23}$. As mentioned earlier, neutrino beams primarily produce muon neutrinos, so beam neutrino experiments study the disappearance of muon neutrinos and the appearance of electron neutrinos. The muon neutrinos may oscillate into tau neutrinos, however detection of tau neutrinos is even more difficult. For one, the energy required to produce a tau lepton $(3.5 \mathrm{GeV})$ may be higher than the neutrino energy, and the lifetime of the tau lepton is very short $\left(<3 \times 10^{-13} \mathrm{~s}\right)$, making detection of the tau lepton difficult. Nuclear reactors produce only electron antineutrinos, so reactor neutrino experiments can determine the solar mixing angle $\theta_{13}$ by searching for electron antineutrino disappearance. Since the energies of reac- 
tor neutrinos are too low (MeV-range) to produce muons or tau leptons, the study of muon/tau neutrino appearance is not possible. Daya Bay [54] determined $\theta_{13}$ to high precision. The current values of the mixing angles and mass splittings are given in Table 2.3.

Before completing the discussion of neutrino oscillations, it is worth noting two classes of oscillation anomalies which can be explained by the existence of a fourth, "sterile" neutrino. The first comes from reactor experiments and is called the "reactor anomaly." Several reactor experiments reported a deficit of electron antineutrinos from nuclear reactors. This deficit may be explained by an incorrect calculation of the neutrino flux or systematic uncertainties, or it can be explained by the oscillation of electron antineutrinos into a sterile antineutrino which is undetectable. The PROSPECT experiment has already studied this anomaly, and its findings indicate the existence of sterile neutrinos is unlikely [55]. The other anomaly comes from beam neutrino experiments. Both the LSND and MiniBooNE experiments reported an excess of electron neutrino events. LSND and MiniBooNE were placed in neutrino beams comprised of mostly muon (anti)neutrinos. Both detectors work by observing Cherenkov light and were placed at locations where $L / E$ is the same. As with the reactor anomaly, these anomalies can be explained by the existence of sterile neutrinos, or incorrect knowledge of backgrounds [28]. This latter anomaly will be tested in MicroBooNE and the SBN program, discussed in Sec. 3.4.1.

Sterile neutrinos differ from the other three neutrinos (called "active") in that they do not interact by any force except for gravity. Since the decay width of the Z boson limits the number of active neutrinos to three, any additional neutrinos must be sterile or much more massive. Despite the fact that they don't interact via the weak force, it is possible to test for the existence of sterile neutrinos, as discussed 
later in Sec. 3.4.2. Further discussion of sterile neutrinos can be found in [56].

\subsection{Neutrino Mass}

The discovery of neutrino oscillations proved that neutrinos have mass, but the absolute masses of the mass states are unknown. From cosmology, we know the the sum of the neutrino masses must be less than $0.170 \mathrm{eV} / \mathrm{c}^{2}$, although these limits are dependent on the validity of the standard cosmological model. Oscillation experiments tell us only the differences of the squares of mass states, so it is not possible to tell which mass state is the heaviest.

Since there are three neutrino masses, there are three mass splittings: $\Delta m_{21}^{2}=$ $m_{2}^{2}-m_{1}^{2}, \Delta m_{32}^{2}=m_{3}^{2}-m_{2}^{2}$ (often called the "atmospheric" mass splitting) and $\Delta m_{31}^{2}=m_{3}^{2}-m_{1}^{2}$ (called the "solar" mass splitting), but only two are independent as $\Delta m_{31}^{2}=\Delta m_{21}^{2}+\Delta m_{32}^{2}$. While it is known that $m_{2}>m_{1}$, and the magnitudes of the splittings are known, it is not known whether $m_{3}>m_{2}$, called normal hierarchy (NH) or $m_{2}>m_{3}$, called inverted hierarchy $(\mathrm{IH})$, however experiments indicate normal hierarchy is favored $[1,2,33]$.

In addition to not knowing what the masses of the neutrinos are, we also do not know how neutrinos get their mass. To begin to see the problem, we can start with a general mass Lagrangian:

$$
-2 \mathscr{L}_{M}=\frac{1}{2}\left(\bar{\psi}, \bar{\psi}^{c}\right)\left(\begin{array}{cc}
m_{D} & m_{M} \\
m_{M}^{*} & m_{D}
\end{array}\right)\left(\begin{array}{l}
\psi \\
\psi^{c}
\end{array}\right),
$$

where $m_{D}$ and $m_{M}$ are called the Dirac and Majorana mass terms, respectively. To obtain a mass, we can diagonalize the matrix. The eigenvalues are $m_{D} \pm\left|m_{M}\right|$. If $m_{M}=0$, the matrix is diagonal to begin with, and the eigenvectors $\psi$ and $\psi^{c}$ are not charge conjugation states. We are left with a Dirac particle, which obtains its mass from the Higgs mechanism. If this is not the case, we are left with Majorana 
neutrinos, which obtain their mass by different means. One theory for the source of neutrino mass is called the "see-saw mechanism" [29,57].

\subsection{Neutrino Detection}

Detecting neutrinos is one of the most difficult parts of neutrino physics. Neutrinos only interact via the weak force; therefore, the interaction cross-section is small. While it is true that cross sections increase as energy increases, as shown in Figure 2.2, even the highest cross sections shown in the Figure are well below that of electron scattering. When a neutrino does interact in a detector, all that is seen is some of the products of that reaction. When charged particles travel in a medium, they ionize atoms along the way, making their existence and path detectable. Neutrinos are neutral, so their trip though matter is invisible.

The way to alleviate this problem is to use an intense source of neutrinos and/or a large detector. Nuclear reactors and particle accelerators work well as they a have large neutrino flux. Large detectors are used since they provide many opportunities for incoming neutrinos to interact. There are a variety of neutrino detectors currently operating. Some, like Super-K, use a large tank of liquid surrounded by

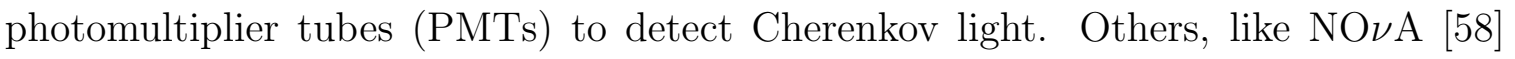
and PROSPECT [59] use scintillator material and PMTs or other light detectors. Unlike Super-K, NO $\nu \mathrm{A}$ and PROSPECT are segmented detectors which allows them to resolve several sources of activity, but not all of the detector volume is active. A third detector is the liquid argon time projection chamber, which has the advantage of being fully active while still providing the advantages of a segmented detector.

\subsection{Beyond the Standard Model and Millicharged Particles}

While the Standard Model correctly describes much of what is known about particle physics, it is not a complete description of the Universe. The Standard 
Model sets the mass of neutrinos at zero, with no oscillation between the flavors, but experiment has shown otherwise. The Standard Model says nothing about the nature of dark matter, which makes up around a quarter of the Universe, nor does it say anything about dark energy, which makes up $70 \%$ of the Universe. We live in a matter, and not antimatter dominated universe, but equal amounts of matter and antimatter were produced in the early Universe, providing yet another mystery.

There are many theories of Beyond the Standard Model (BSM) physics which seek to explain these and other puzzles of the universe, or seek to provide an explanation of anomalous results in particle physics and astronomy. The vast majority of these are beyond the scope of this thesis. Sections 2.5 and 2.6 touched on two of these topics, namely neutrino oscillation, mass and the possible existence of sterile neutrinos. Another topic which will be discussed in the following is millicharged particles.

All free particles in the Standard Model have a charge which is an integer multiple of the charge of the electron. While quarks have fractional charges, they are always found in bound states where the total charge is an integer. It is possible, however to develop a theory where free particles exist with charges much smaller than that of the electron. Such particles are called "millicharged particles."

Millicharged particles arise out of a theory of a dark sector with a $\mathrm{U}(1)^{\prime}$ gauge symmetry. In this dark sector, there exists a massless photon which couples to our photon, a process called kinetic mixing, but with a small coupling constant. The result is that dark sector particles, which have integer charge in their world, appear to us as having fractional charge. The Lagrangian for such particles interacting in the standard electromagnetic field is:

$$
L=i \bar{\chi} \gamma^{\mu} \partial_{\mu} \chi-m_{\chi} \bar{\chi} \chi+Q_{\chi} A_{\mu} \bar{\chi} \gamma^{\mu} \chi
$$


where $m_{\chi}$ is the Dirac mass of the particle, $Q_{\chi}$ is the charge, $A_{\mu}$ is the ordinary photon, $\gamma^{\mu}$ are the Dirac matrices [60].

Millicharged particles have been proposed as a solution to the EDGES anomaly and may constitute up to $2 \%$ of the dark matter in the Universe. The parameter space of these particles is constrained by astrophysical events, for example supernova 1987A, big bang nucleosynthesis and by experiments, for example at SLAC and the LHC [61].

These particles can be produced in a particle accelerator by striking a proton beam onto a fixed target. The millicharged particles arise via the decays of neutral mesons or direct Drell-Yan pair production arising from proton interactions in the target $[21,62]$. A Feynman diagram of a pair of millicharged particles produced in a $\pi^{0}$ decay is shown in Figure 2.13. This production process is similar to that of beam neutrinos discussed in Section 2.3, except that neutrinos arise from charged, not neutral mesons. Indeed, a fixed target producing neutrinos can also produce millicharged particles. While the magnetic horns focus the charged mesons, thereby constraining the width of the neutrino beam, the horns have no effect on the neutral mesons and little effect on the millicharged particles, so the beam of millicharged particles is much wider.

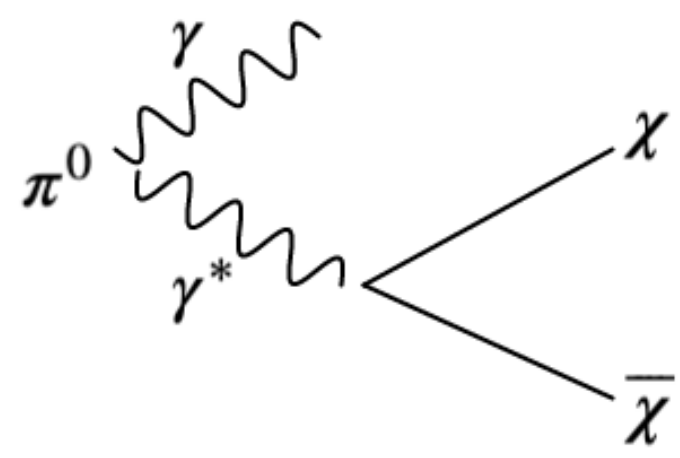

Figure 2.13. Feynman diagram of a pair of millicharged particles produced by the decay of a $\pi^{0}$ meson. 
Several authors have proposed ways to search for these particles $[21,60,63]$. Chapter 6 presents the results of a search for millicharged particles using a liquid argon time projection chamber. 
Table 2.1. Masses and Charges of Particles in the Standard Model. Neutrinos are treated as massless in the SM. Charge is given in units of the charge of the electron. Values from [1].

\begin{tabular}{|c|c|c|}
\hline Particle & Mass $(\mathrm{MeV})$ & Charge \\
\hline$u$ & 2.2 & $2 / 3$ \\
\hline$d$ & 4.7 & $-1 / 3$ \\
\hline$c$ & 1280 & $2 / 3$ \\
\hline$s$ & 96 & $-1 / 3$ \\
\hline$t$ & 173000 & $2 / 3$ \\
\hline$b$ & 4180 & $-1 / 3$ \\
\hline$\nu_{e}$ & 0 & 0 \\
\hline$\nu_{\mu}$ & 0 & 0 \\
\hline$\nu_{\tau}$ & 0 & 0 \\
\hline$e$ & 0.511 & -1 \\
\hline$\mu$ & 106 & -1 \\
\hline$\tau$ & 1780 & -1 \\
\hline
\end{tabular}


Table 2.2. Generations of the Standard Model. From [2]

\begin{tabular}{ccc}
\hline \hline First Generation & Second Generation & Third Generation \\
\hline$u$ & $c$ & $t$ \\
$d$ & $s$ & $b$ \\
$\nu_{e}$ & $\nu_{\mu}$ & $\nu_{\tau}$ \\
$e$ & $\mu$ & $\tau$ \\
\hline
\end{tabular}

Table 2.3. Current best-fit values for neutrino mixing angles, mass splittings and CP violating phase. From [1].

\begin{tabular}{cc}
\hline \hline Parameter & Value \\
\hline$\Delta m_{21}^{2}$ & $7.37 \times 10^{-5} \mathrm{eV}^{2}$ \\
$\Delta m_{31}^{2}$ & $2.56 \times 10^{-3} \mathrm{eV}^{2}$ \\
$\sin ^{2} \theta_{12}$ & 0.297 \\
$\sin ^{2} \theta_{23}(\mathrm{NH})$ & 0.425 \\
$\sin ^{2} \theta_{23}(\mathrm{IH})$ & 0.589 \\
$\sin ^{2} \theta_{13}(\mathrm{NH})$ & 0.0215 \\
$\sin ^{2} \theta_{13}(\mathrm{IH})$ & 0.0216 \\
$\delta / \pi$ & 0.38 \\
\hline
\end{tabular}




\section{CHAPTER 3 \\ THE LIQUID ARGON TIME PROJECTION CHAMBER}

The three particle detectors commonly used in neutrino experiments are liquid scintillator detectors, Cherenkov detectors and liquid argon time projection chambers (LArTPCs). Cherenkov detectors such as Super-K, SNO and MiniBooNE consist of a tank of liquid, usually water, surrounded by light detectors, usually PMTs. The detectors operate by detecting Cherenkov light emitted as particles travel through the detector medium. Cherenkov light is emitted when a particle travels faster than the speed of light in the medium. The result is an electromagnetic shockwave, similar to the one produced when an object travels faster than the speed of sound in a medium. The pattern of light is different for different particles, allowing the detector to distinguish between particle species. The notable exception is electrons and photons, which appear the same to a Cherenkov detector [64].

Scintillation detectors consist of cells optically separated from each other and filled with liquid scintillator. A PMT or other light detector is attached to each cell to observe the scintillation light produced when a particle passes through the cell. By examining the light pulses, it is possible to distinguish between different particles, a technique called "pulse shape discrimination." This technology, used by NO $\nu \mathrm{A}$ and PROSPECT, has the advantage of rapid readout. A disadvantage is that it must be segmented, so not all of the detector is active [64].

The liquid argon time projection chamber (LArTPC) is the third currently popular technology for neutrino experiments. One if its main advantages is its high resolution in 3D. The ability to zoom in on particle activity, down to a few millimeters, is essential when one wishes to detect low energy particles which travel a short 
distance. There are other detectors which have as good or even better resolution, for example bubble chambers, but LArTPCs have several advantages. For one, they provide calorimetric information, a necessary component for particle identification. LArTPCs are also easily "reset," meaning the detector is back to its initial state soon after the event occurred. LArTPCs record particle tracks in 3D, compared to 2D for bubble chambers. In addition, LArTPCs can be scaled to large sizes, where again the volume is fully active.

A noble liquid/gas must be used in TPC experiments so electrons ionized by neutrino interaction final-state particles do not attach to the medium. Noble liquids other than argon may be chosen as the detector medium, but argon has several advantages. First, it is inexpensive, so scaling to large sizes is cost-effective. Liquid nitrogen can be used to keep the argon at liquid temperatures $(87 \mathrm{~K})$, which is also cost-effective. At $\sim 1.4 \mathrm{~g} / \mathrm{cm}^{3}$, liquid argon is dense, increasing the likelihood of and number of neutrino interactions. Finally, argon is transparent to its own scintillation light.

LArTPC technology is currently in use in the MicroBooNE detector at Fermilab and the protoDUNE [65] detector at CERN. Previous LArTPCs, such as ArgoNeuT [15], ICARUS [66] and LArIAT [67], have demonstrated this technology's potential, and several other LArTPCs will operate in the future, such as SBND [28] and DUNE [27], in addition to the reuse of the ICARUS detector at Fermilab [28]. The use of the LArTPC in DUNE ensures this technology will play a central role in neutrino physics for decades to come. While the operation of all LArTPCs is similar, this chapter uses examples mainly from ArgoNeuT, as it is the detector used for this thesis.

In addition to describing this detection technology, this chapter also describes the propagation of particles through liquid argon, focusing on photons and neutrons, 
and provides the background for the low-energy photon study discussed in subsequent chapters. This chapter also describes how millicharged particles propagate through liquid argon.

\subsection{Principle of Operation}

The principle of operation of a LArTPC is as follows. As charged particles traverse the liquid argon medium, they ionize electrons at a rate of about 42,000 electrons/MeV. A cathode biased at a high voltage, typically on the order of $10^{5} \mathrm{~V}$, along with a field shaping cage, create a uniform electric field. This electric field causes the ionized electrons to drift at a constant velocity to a series of sensitive wire planes (usually two or three). Figure 3.1 shows the layout of these components and how a neutrino interaction event might be reconstructed. The wire spacing (pitch) within each plane is typically between 3-5 mm with the wires in each plane oriented at an angle with respect to the other planes. Ionized electrons first encounter two induction planes. The wires on these planes detect the electrons as they approach and travel away from the wires, yielding a bipolar waveform. After passing the induction planes, the electrons are collected on the collection plane, where the waveform is unipolar.

In each detector readout, each wire signal is sampled at a rate of a few megahertz. When a LArTPC is used in a neutrino or charged particle beam, the total readout time is typically longer than the maximum drift time. This is done to examine activity occurring before and after the beam arrives and to account for the spread of arrival times for beam particles. The black line in Figure 3.11 shows the signals recorded on one induction plane wire (top) and one collection plane wire (bottom) in the ArgoNeuT TPC (see Sec. 3.3). The wire signals for a triggered detector readout are collected into an event. The wire signals are then processed in an automated reconstruction (see Sec. 3.5). 


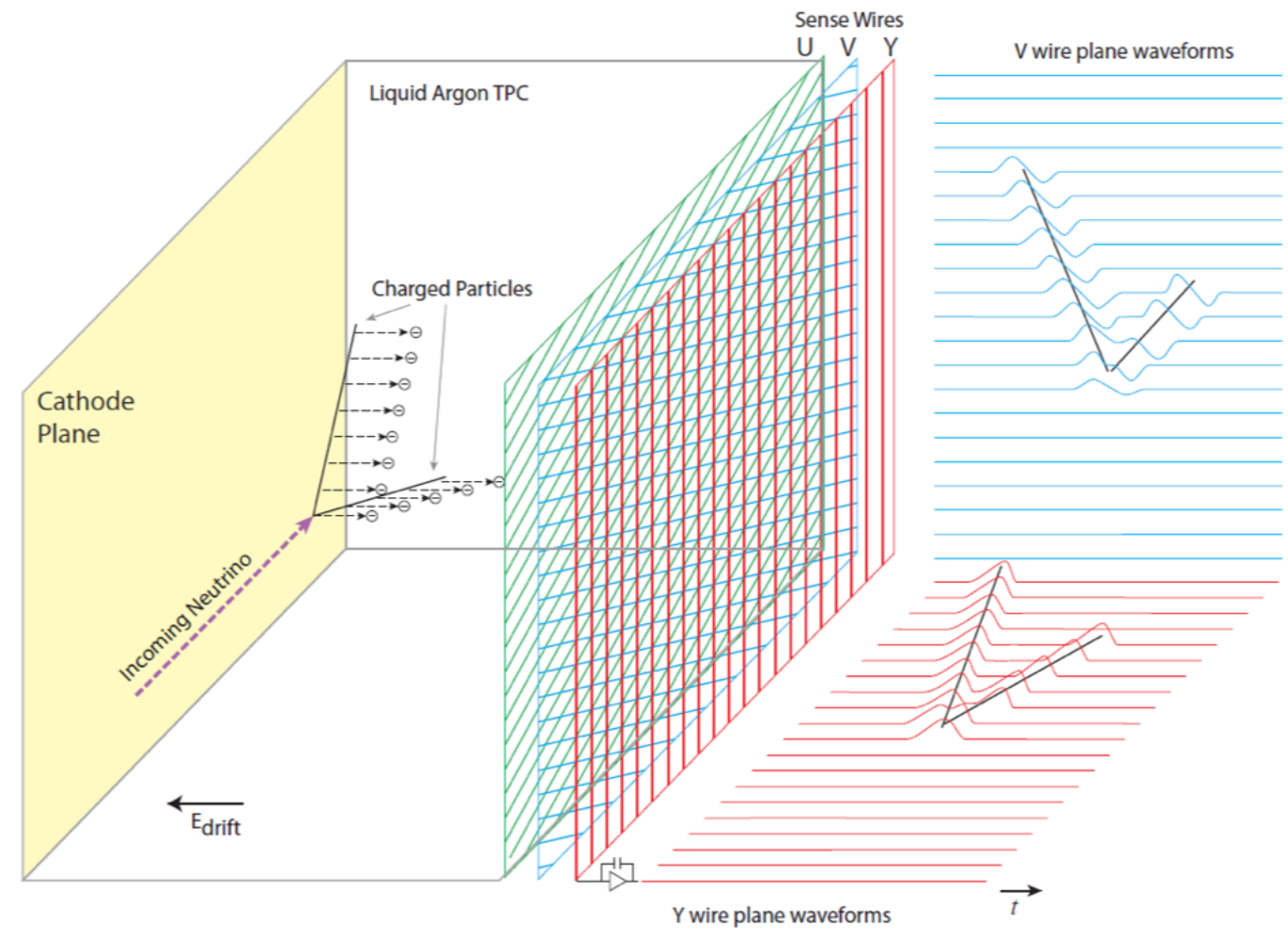

Figure 3.1. Diagram of LArTPC operation. As charged particles travel through the liquid argon, electrons are ionized. A cathode (left) biased at a high voltage creates an electric field which causes the ionized electrons to drift to planes of wires (middle) which detect the electrons. Wave forms on wires are read out, and the activity of the charged particles is reconstructed (right). No light collection system is shown here, however it is typically placed behind the wire planes. Image from $[12]$.

Scintillation light is also produced as a charged particle travels through the argon and ionizes or excites the argon. Ionized argon atoms can combine with other nearby argon atoms, producing an excited state. An excited atom returns to its ground state by the emission of photons. To detect this light, a light collection system, typically PMTs, is placed behind the wire planes. In argon, the emitted light has a wavelength of $128 \mathrm{~nm}(9.39 \mathrm{eV})$ with $\sim 10^{4}$ photons $/ \mathrm{MeV}$ emitted. This is in the vacuum ultraviolet (VUV) range and is invisible to commonly used light collection systems, so wavelength-shifting compounds are applied to the PMTs to turn the VUV light into visible light [27]. 
The scintillation light is mainly used to trigger the detector readout. As scintillation light arrives at the PMTs almost immediately (ns) after it is emitted, wire readout can begin as soon as a neutrino interaction occurs. This allows for an accurate determination of the drift coordinate. Since the detection of scintillation light indicates the presence of particle activity in the event, only events with scintillation light (and therefore activity) are read out. This reduces the number of "empty" or "neutrino-free" events and lowers the amount of data storage space required.

\subsection{Particle Propagation Through Argon and Detection}

After a neutrino interacts, neutral and charged particles are produced and subsequently travel through matter. By obtaining information about these secondary particles, such as their multiplicity, type and energy, it is possible to reconstruct the neutrino interaction. In order to determine this information about the secondary particles, it is necessary to know how they propagate through matter. The behavior of charged particles and low energy $(<10 \mathrm{MeV})$ photons, neutrons and millicharged particles, which are the subject of this thesis, is described below.

3.2.1 Charged Particles. Fast moving charged particles lose energy in collisions typically $<100 \mathrm{eV}$ which ionize or excite atoms in the medium. For particles more massive than the electron and traveling at moderately relativistic speeds, the average rate of energy loss, $d E / d x$ is given by the Bethe equation:

$$
\left\langle-\frac{d E}{d x}\right\rangle=K z^{2} \frac{Z}{A} \frac{1}{\beta^{2}}\left(\frac{1}{2} \ln \frac{2 m_{e} c^{2} \beta^{2} \gamma^{2} W_{\max }}{I^{2}}-\beta^{2}-\frac{1}{2} \delta(\beta \gamma)\right),
$$

where $K$ is a constant, $z$ is the charge number of the incident particle, $Z$ is the atomic number for the medium, $A$ is the atomic mass for the medium, $\beta=v / c, m_{e}$ is the mass of the electron, $c$ is the speed of light, $\gamma$ is the relativistic gamma, $W_{\max }$ is the maximum energy transferred to an electron in a single collision, $I$ is the mean excitation energy and $\delta(\beta \gamma)$ is a density effect correction. Multiplying the Bethe 
equation by the density of the medium gives the linear stopping power, or the energy loss per unit length.

Although $\langle d E / d x\rangle$ is used frequently, it does have drawbacks, namely the average is weighed by a few very high energy collisions. It is sometimes better to use the most probable value of $d E / d x$ :

$$
\left.\frac{d E}{d x}\right|_{\text {m.p. }}=\xi\left(\ln \frac{2 m_{e} c^{2} \beta^{2} \gamma^{2}}{I}+\ln \frac{\xi}{I}+j-\beta^{2}-\delta(\beta \gamma)\right),
$$

where $\xi=\left(K Z z^{2} x\right) /\left(2 A \beta^{2}\right), x$ is the thickness of the liquid argon cell and $j$ is a constant. More information can be found in [1].

3.2.2 Photons. As shown in Figure 3.2, at energies below $10 \mathrm{MeV}$, photons interact in argon primarily by Compton scattering. As the energy of the photon decreases, photoelectric absorption contributes more to the total cross section. In both of these interactions, a single electron is produced. When the energy of the photon is above $1.022 \mathrm{MeV}$, pair production is possible, with the fraction of the total cross section increasing with energy. In pair production, an electron and positron, each with mass $0.511 \mathrm{MeV}$, are produced. Produced electrons then ionize/excite argon similarly to heavy charged particles, creating a detectable signal in the LArTPC.

3.2.3 Neutrons. Neutrons primarily interact in two ways in argon: by scattering off a nucleus or by being captured by a nucleus. The scattering can be elastic or inelastic. In elastic scattering, a neutron scatters off a nucleus without transferring energy to it. In inelastic scattering, the neutron transfers energy to the nucleus, leaving it in an excited state. In order to inelastically scatter, the neutron must have a kinetic energy which is at least that of the first excited state in the nucleus. The nucleus then deexcites, releasing photons in the MeV-range. If the energy transferred to the nucleus is large enough, the nucleus may release nucleons as well. When the kinetic energy of a neutron is low enough, it can be captured by a nucleus. This also causes the new 


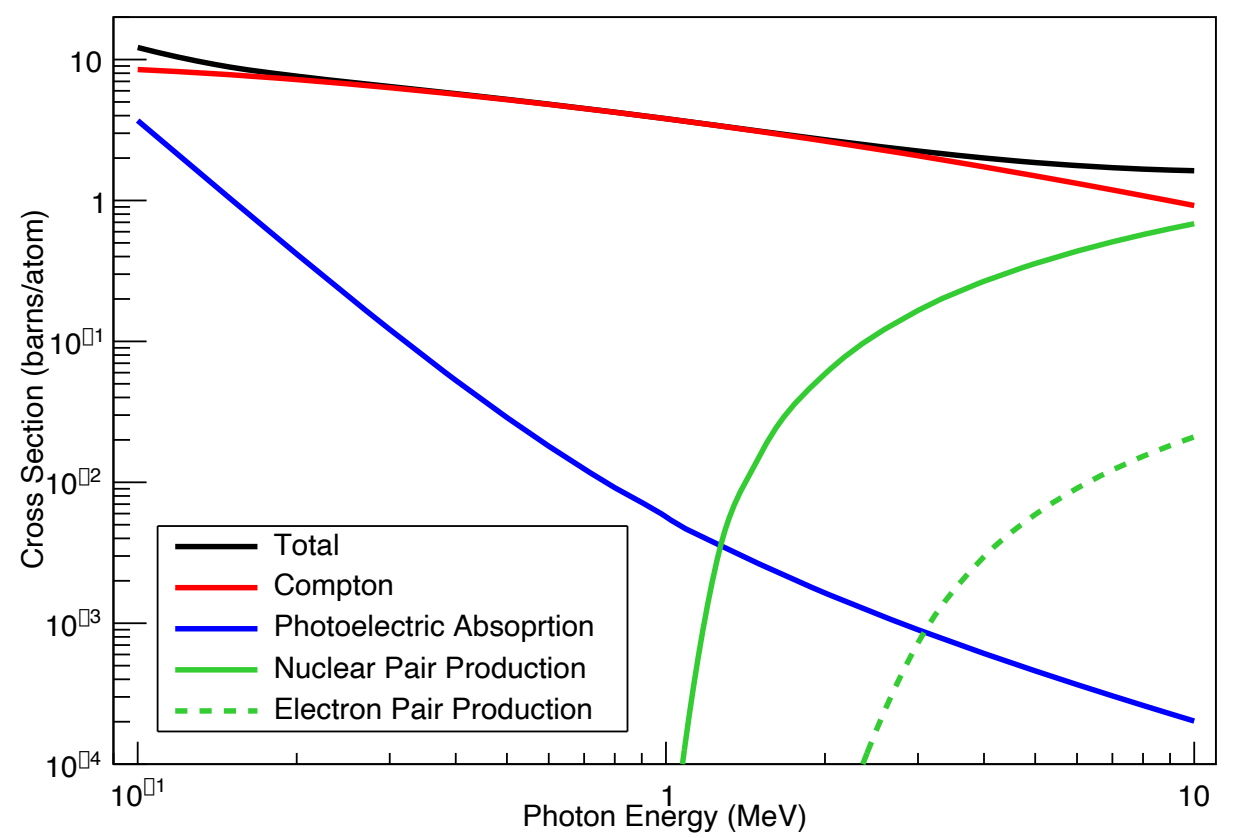

Figure 3.2. Cross-sections of photon interactions in liquid argon for energies between $0.1-10 \mathrm{MeV}$. Data from [13].

nucleus to release $\mathrm{MeV}$-scale photons. Figure 3.3 shows measured cross-sections of various neutron interaction processes. Inelastic scattering with emission of photon(s) is the most likely process for neutrons below a few tens of $\mathrm{MeV}$, producing photons with a range of energies, with the minimum being at $1.46 \mathrm{MeV}$, the first excited state of ${ }^{40} \operatorname{Ar}[68]$.

I performed a GEANT4 simulation to study the propagation of neutrons in liquid argon. This simulation used the NeutronHP physics list with a volume of liquid argon of $80 \mathrm{~m}^{3}$ inserted into GEANT. Two samples of 10,000 monoenergetic neutrons were studied. One contained neutrons with a kinetic energy of $100 \mathrm{keV}$. This energy is too low for inelastic scattering as the first excited state of ${ }^{40} \mathrm{Ar}$ is $1.46 \mathrm{MeV}$, so all photons produced in this sample will come from neutron capture. The second sample contained neutrons with a kinetic energy of $10 \mathrm{MeV}$, an energy high enough to reach the highest excitation levels of ${ }^{40} \mathrm{Ar}$. 


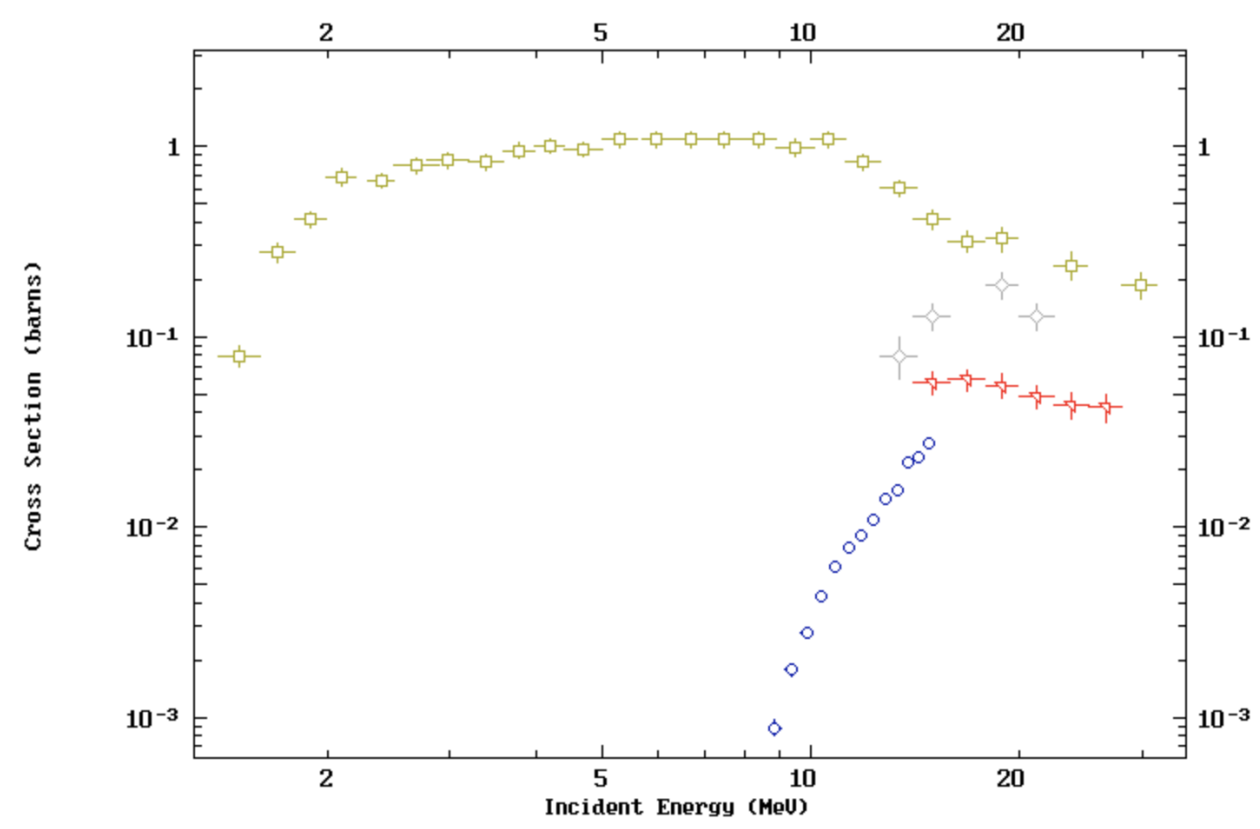

Figure 3.3. Cross-sections for inelastic scattering with photon production (gold square), proton emission (blue circle), and neutron emission (red triangles and gray diamonds). Image from [14].

The energies of individual photons by neutron interactions are given in Figure 3.4. The left plot shows the energies of individual photons from neutron capture, while the right plot mainly shows the energies of photons from inelastic scattering, with some due to neutron capture. We see that in both cases, the energies of the photons are below $10 \mathrm{MeV}$, and that certain energies are more common. In particular, the peak at $1.46 \mathrm{MeV}$ is particularly prominent in the right plot, indicating that deexcitation from the first excited state of ${ }^{40} \mathrm{Ar}$ is common. The total amount of energy, released to photons is variable in scattering events depending on the energy of the incoming neutrino. In capture events, however, the total energy is almost always $\sim 6.1 \mathrm{MeV}$. Figure 3.5 shows the total energy released in photons by neutron capture events (left), and inelastic scattering and neutron capture (right). Thus, MeV-scale reconstruction in liquid argon is essential to identifying and reconstructing final-state neutrons from neutrino interactions. 

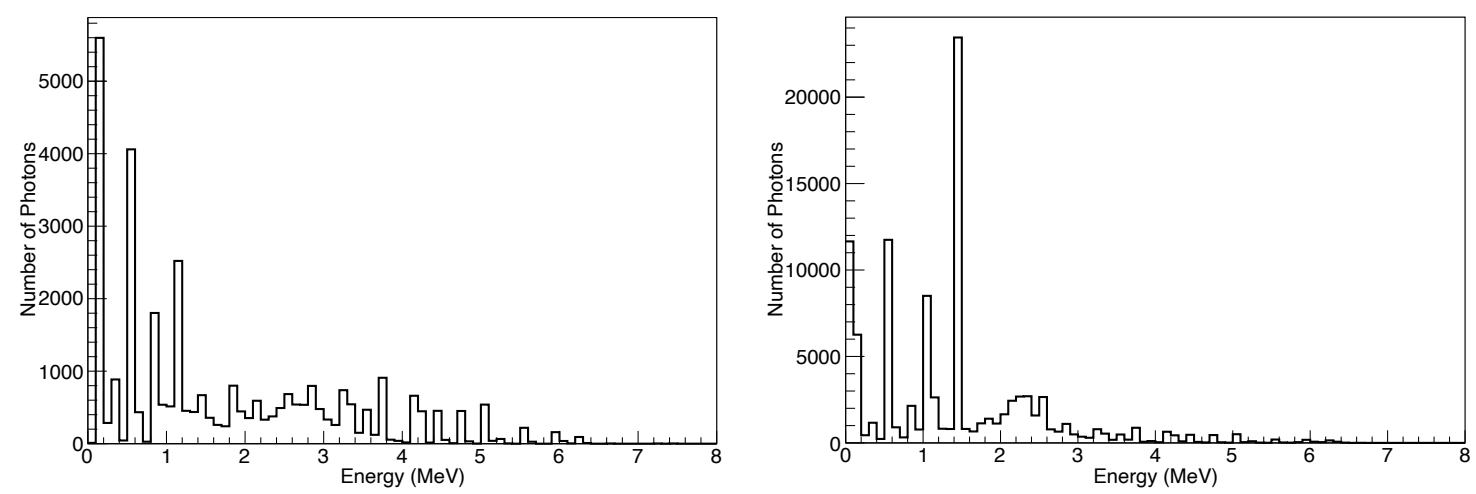

Figure 3.4. Energies of individual photons from $100 \mathrm{keV}$ neutrons (left) and $10 \mathrm{MeV}$ neutrons (right). Note the peak at $1.46 \mathrm{MeV}$ due to the first excited state of ${ }^{40} \mathrm{Ar}$ in the right plot.
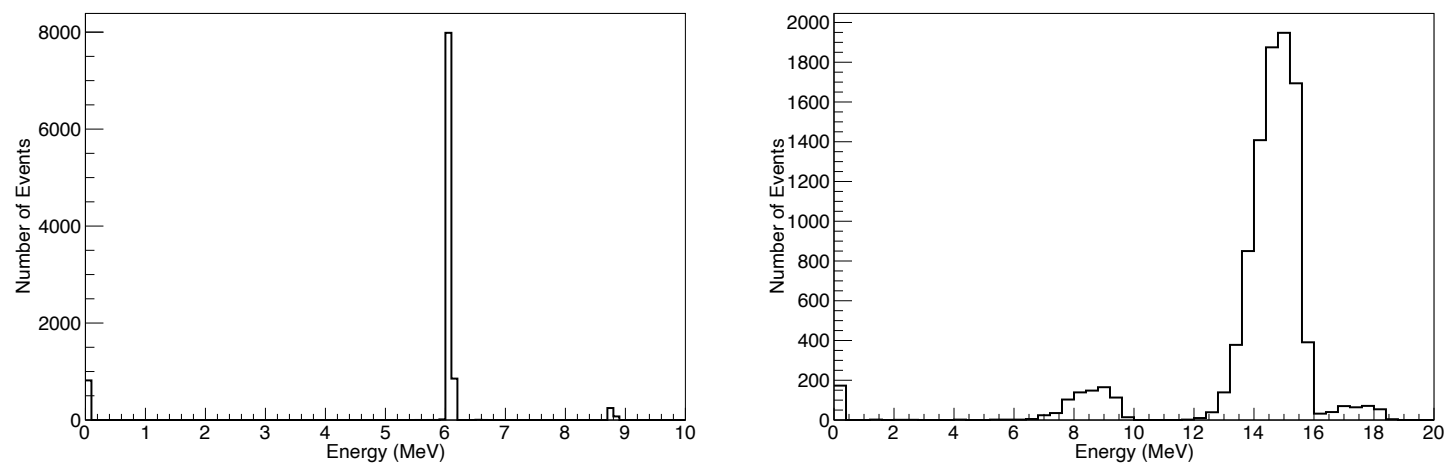

Figure 3.5. Total energy of photons from $100 \mathrm{keV}$ neutrons (left) and $10 \mathrm{MeV}$ neutrons (right). The left plot shows photons only from neutron capture, while the right plot shows photons from neutron capture and inelastic scattering.

3.2.4 Millicharged Particles. Like all charged particles, millicharged particles lose energy by atomic excitation and ionization. Millicharged particles also obey the Bethe equation (Eq. 3.1), and because their charge ( $z$ in Eq. 3.1) is smaller, the ionization and excitation rates are reduced by $\epsilon^{2}$. Consequently, the majority of the $\mathrm{mCP}$ ionization track is undetectable in most current detectors. As discussed in Sec. 3.2.1, occasionally a particle traveling through matter will strike an electron hard enough such that the recoil electron produces produce a visible signal via subsequent ionization, similar to delta rays produced by a muon. The distribution of recoil 
energies scales with the inverse squared of the electron recoil energy,

$$
\frac{d \sigma}{d E_{r}} \simeq \frac{2 \pi \alpha \epsilon^{2}}{m_{e} E_{r}^{2}},
$$

where $\sigma$ is the cross-section, $E_{r}$ is the energy of the recoil electron, $\alpha$ is the fine structure constant, and $\epsilon$ is the fractional $\mathrm{mCP}$ charge, and we have taken the relativistic mCP limit. Low-energy thresholds are therefore key to detecting these "delta rays" produced by mCPs.

\subsection{The ArgoNeuT LArTPC Experiment at Fermilab}

The first LArTPC neutrino experiment in the U.S. was ArgoNeuT, a quarterton LArTPC. ArgoNeuT has been used primarily to study neutrino-argon cross sections and final-state production rates using neutrinos from the NuMI beamline at Fermilab for five months in 2009-2010. ArgoNeuT was the detector used for the study in this thesis of low-energy $(<10 \mathrm{MeV})$ photons from neutrino interactions in liquid argon and for a search for millicharged particles produced in the NuMI target.

The TPC, shown in Figure. 3.6, was small, $47 \times 40 \times 90 \mathrm{~cm}^{3}(x, y, z)$, with the longest dimension along the beam. On one side of the $\mathrm{x}$-direction was a cathode made of G10 and copper. On the opposite end were three wire planes, spaced $4 \mathrm{~mm}$ apart. All wires were $152 \mu \mathrm{m}$ in diameter, aligned parallel to the other wires within a plane and spaced $4 \mathrm{~mm}$. The innermost wire plane was the shield plane, made up of 225 wires oriented vertically (in the $y$ direction). These wires were not instrumented but were used to shape the electric field and to provide shielding to the other wire planes against induced signals from drifting charge. The next plane was the induction plane which contained 240 wires oriented at 60 degrees to the $z$-axis. Finally there was the collection plane, which also contained 240 wires and was oriented at -60 degrees to the $z$-axis. The location of the wire planes can be seen in Figure 3.7.

To create the electric field, the cathode was biased a negative high voltage 


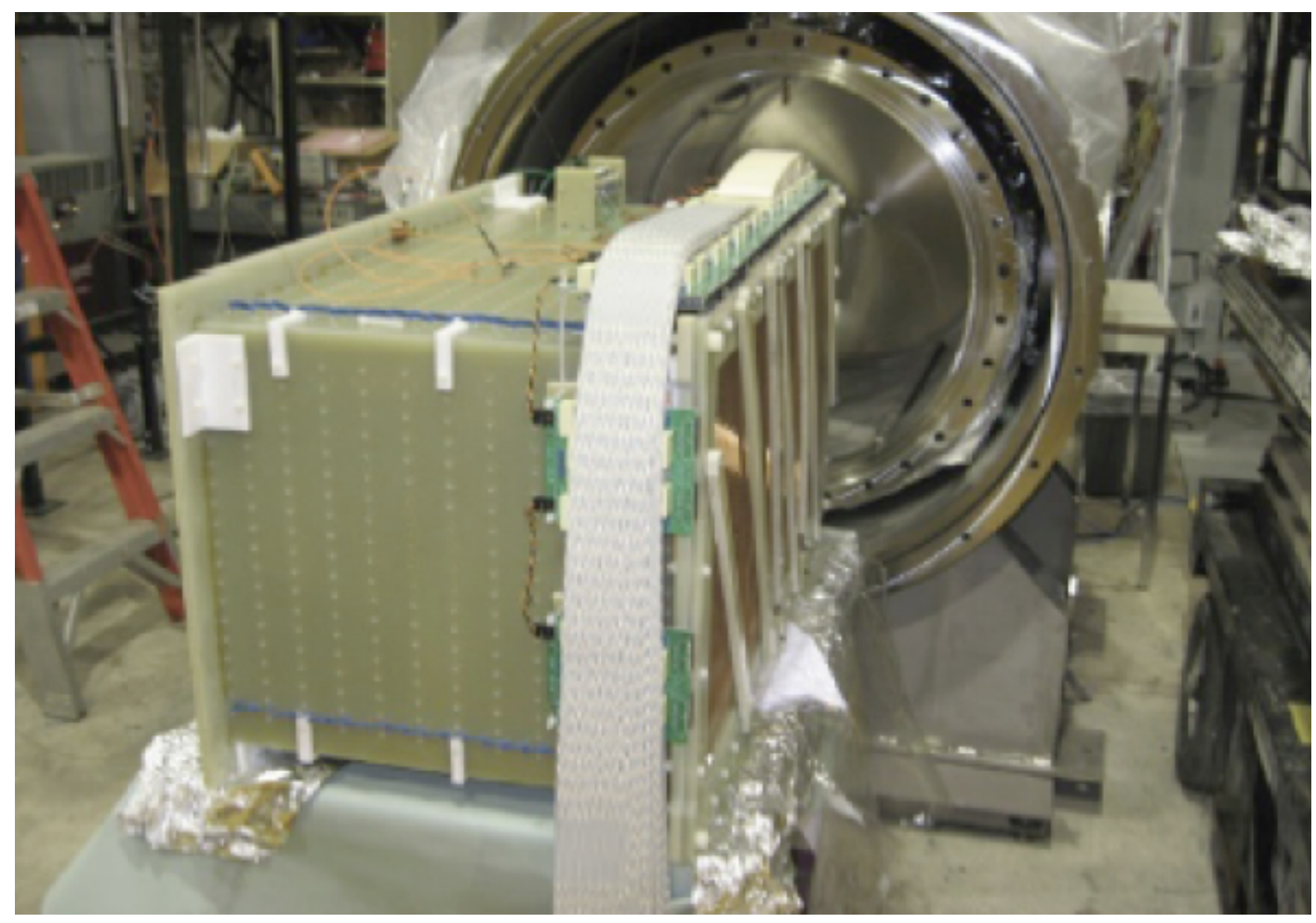

Figure 3.6. Image of the ArgoNeuT TPC (front) and cryostat (back). The wires are seen on the right of the TPC. Image from [15].

of magnitude $23.5 \mathrm{kV}$. On the opposite end were three wire planes, each biased to a different voltage. By correctly setting the potentials of the wires, the wires do not interfere with the drifted charge. In between were copper strips which wrapped around the TPC forming the field shaping system, made of rectangular rings in the $y z$-plane. The potentials of the rings decreased linearly from the cathode to the wire planes. The combination of the cathode, field cage and first wire plane created a uniform electric field pointing towards the cathode and with a magnitude of 481 $\mathrm{V} / \mathrm{cm}$. This caused ionized electrons to drift towards the wire planes at a constant velocity of $1.57 \mathrm{~mm} / \mu \mathrm{s}$, with a total drift time of $300.5 \mu \mathrm{s}$. The cathode and rings are seen in the inset of Figure 3.7.

In each detector readout, each wire channel was sampled every $198 \mathrm{~ns}$, for a total readout window of $405 \mu$ s or 2048 samples. The maximum drift time is shorter 


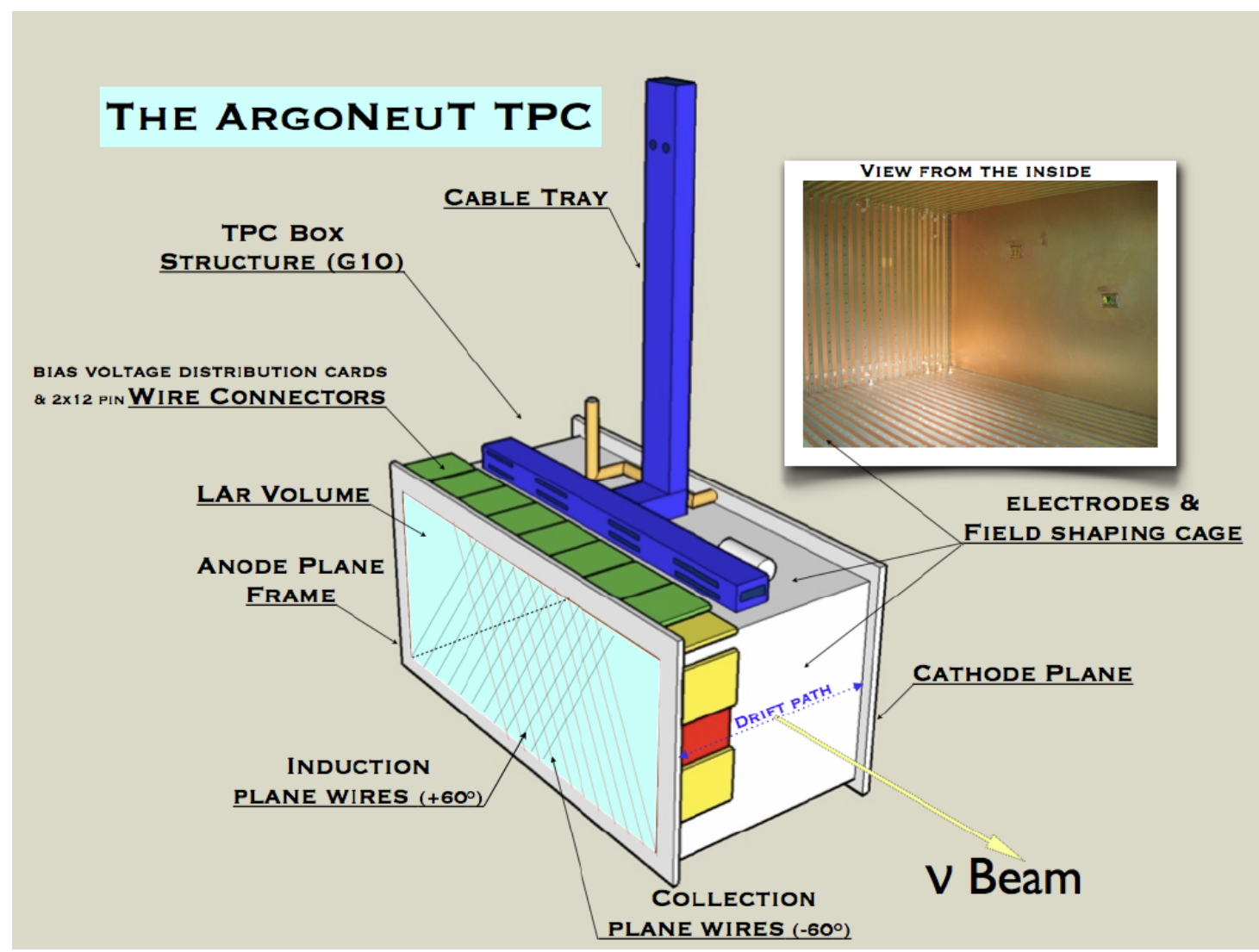

Figure 3.7. Diagram of the ArgoNeuT TPC showing the layout of the different TPC components. The inset shows the cathoe and field shaping rectangular rings. Image from $[15]$.

than this $(300.5 \mu \mathrm{s})$; the extra readout time was used for presampling of events to determine baselines and noise levels. Triggering for an event was determined by the NuMI beam spill, at a rate of $0.5 \mathrm{~Hz}$. While the NuMI beam is intense, ArgoNeuT is a small detector, and neutrino cross sections are low, so most events do not contain a neutrino interaction or other high energy activity. Such events are referred to as "empty" events, although they may contain small amounts of activity due to radioactive decays or electronics noise. A more detailed description and operational parameters of the ArgoNeuT detector are given in [15].

ArgoNeuT's small (4 mm) wire spacing and high readout rate (198 ns sampling time) allow for millimeter precision of $3 \mathrm{D}$ positions within the detector. Wire and 
time coordinates for both planes can be converted into $x, y, z$ coordinates by the following equations:

$$
\begin{gathered}
x=t v_{d} \\
y=(0.4 \mathrm{~cm}) \frac{w-v}{2 \cos \theta} \\
z=(0.4 \mathrm{~cm}) \frac{w+v}{2 \sin \theta}-\frac{h}{2 \tan \theta},
\end{gathered}
$$

where $t$ is the sample time, $v_{d}$ is the drift velocity $(1.57 \mathrm{~mm} / \mu \mathrm{s}, w$ is the collection plane wire number, $v$ is the induction plane wire number, $\theta$ is the angle of the wires (60 degrees), and $h$ is the height of the TPC (40 cm) [15]. The wire planes and coordinate system can be seen in Figure 3.8 .

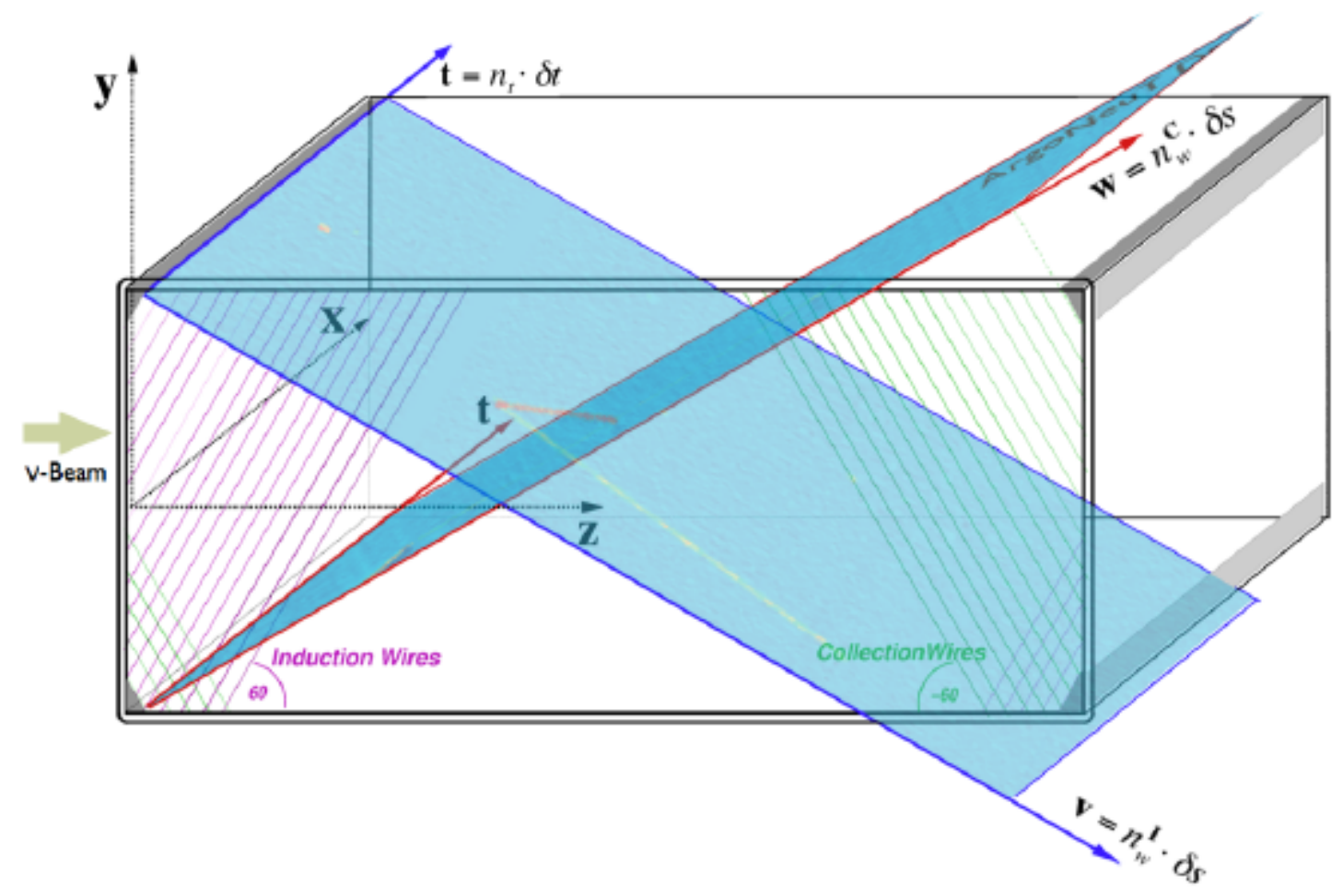

Figure 3.8. ArgoNeuT's coordinate system and wire planes. Image from [15].

ArgoNeuT benefited from the presence of the MINOS near detector (ND) located immediately downstream of it, as shown in Figure 3.9. The MINOS ND [42] was a large (compared to ArgoNeuT) magnetized detector made of alternating layers 
of steel and scintillating strips. As a result, the momenta and signs of muons which were produced by neutrino interactions in ArgoNeuT, exiting the detector and entering the MINOS ND can be determined by using information from the MINOS ND. This is done by matching tracks in ArgoNeuT to tracks in the MINOS ND [15].

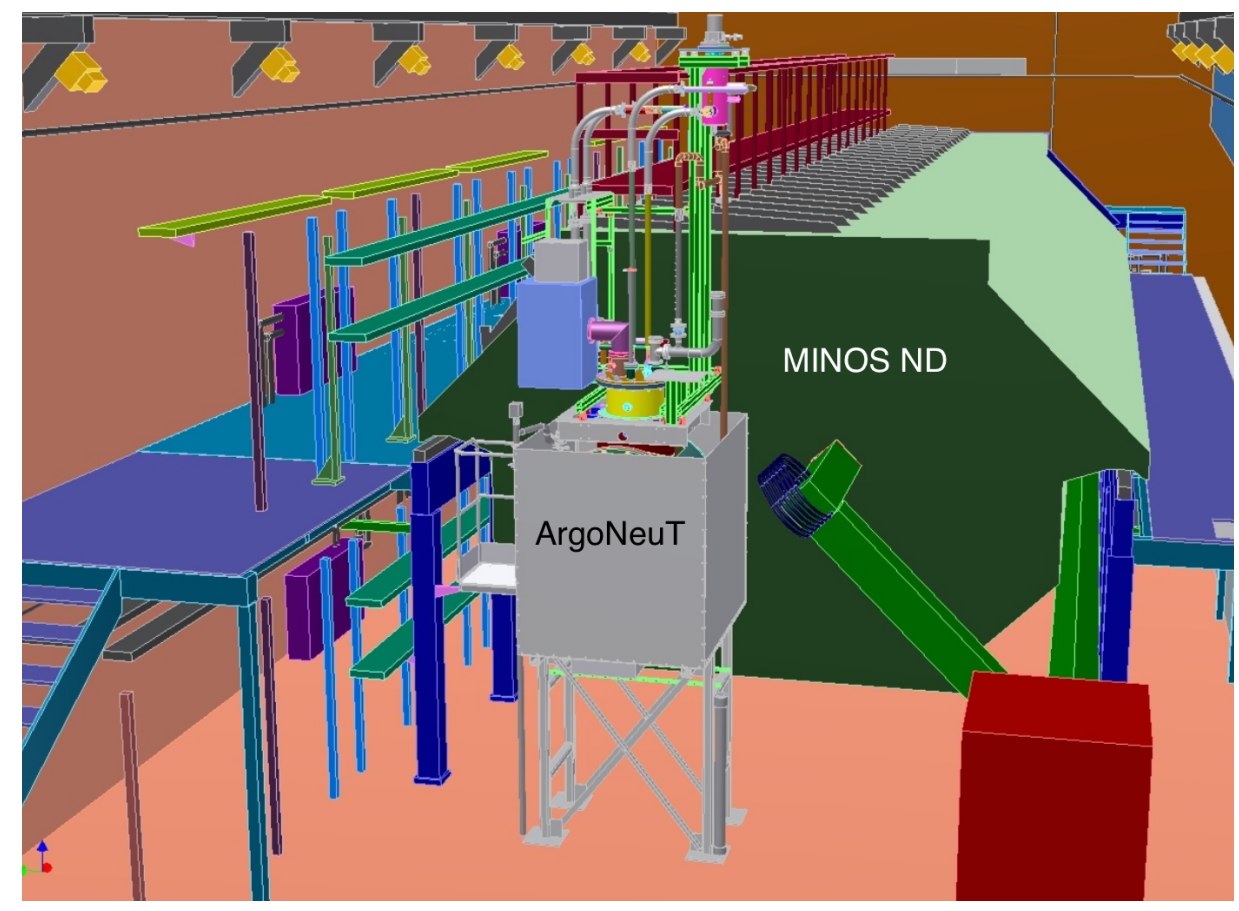

Figure 3.9. Relative locations of ArgoNeuT and the MINOS ND. The neutrino beam points into the page. Image from [15].

There are three benefits to using MINOS ND information. First, by determining the charge of the muon, it is possible to determine whether a muon neutrino or antineutrino is responsible for the CC interaction seen in ArgoNeuT. Second, it is possible to determine the energy of the muon. Since ArgoNeuT was small, all muons produced within ArgoNeuT were energetic enough to escape the detector volume, making determining the muon's energy impossible. The MINOS ND's magnetic field causes the muon to travel in a curved path, and the radius depends on the momentum of the muon, so even if the muon escapes the MINOS ND, it is possible to determine its energy. Third, since only muons are able to penetrate several layers of the steel in the front of the MINOS ND, it is possible to determine whether a track in ArgoNeuT 
was caused by a muon or pion, something that is difficult in LArTPCs. An example of a muon produced within ArgoNeuT, then leaving and entering the MINOS ND is shown in Figure 3.10. Because the MINOS ND is magnetized, the muon's track is curved. By matching the muon track in ArgoNeuT to the muon track in the MINOS ND, ArgoNeuT can obtain additional information about the muon and neutrino interaction. ArgoNeuT also benefited from its placement $100 \mathrm{~m}$ underground; this earth above ArgoNeuT stopped the vast majority of cosmic rays. In ArgoNeuT, activity from cosmic rays is expected in fewer than 1 in 7000 triggers.

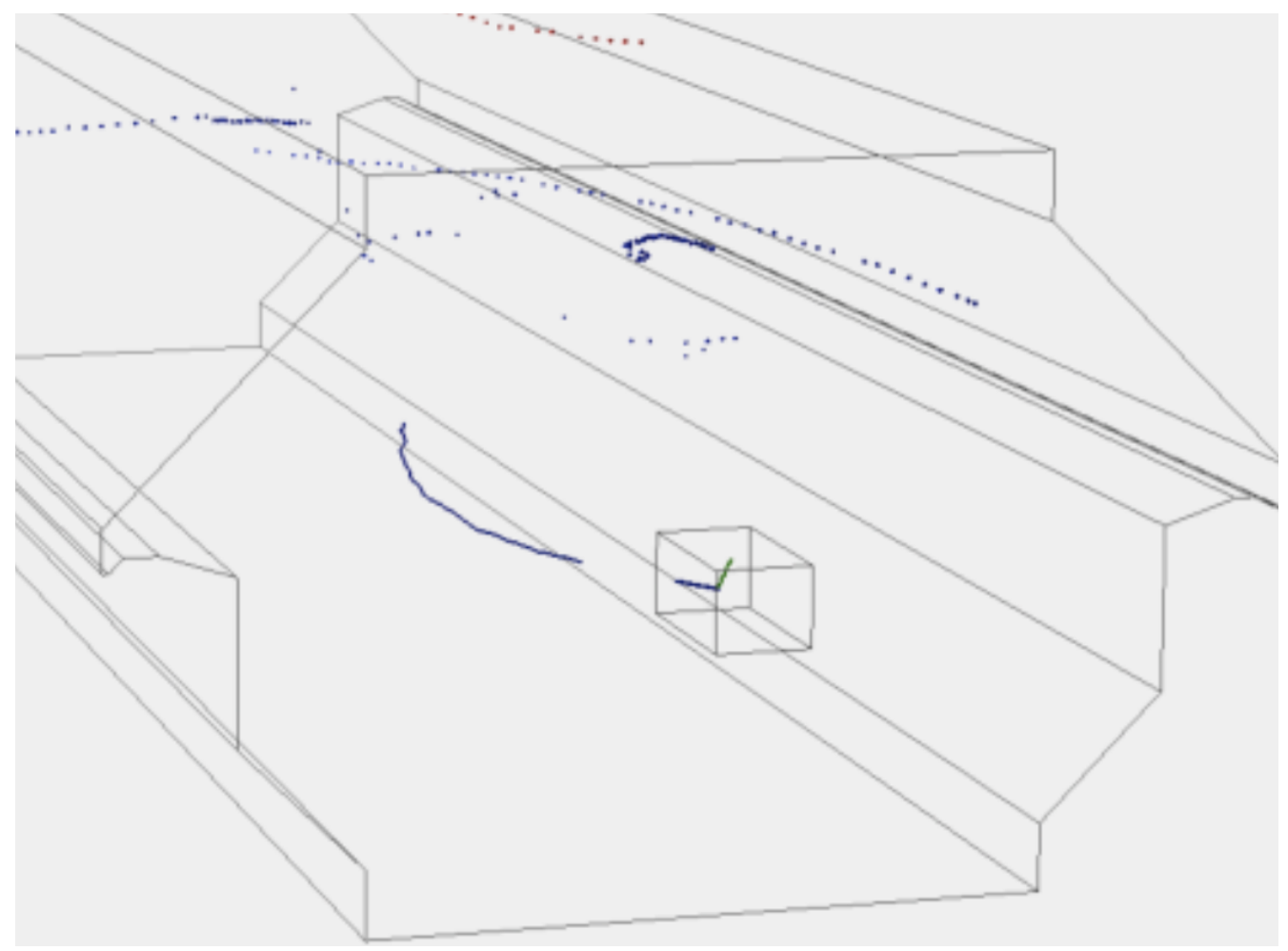

Figure 3.10. Matching of tracks between ArgoNeuT and the MINOS ND. A muon (long track) produced in ArgoNeuT (small box in foreground) leaves ArgoNeuT and enters the MINOS ND (large octagon in background). Also shown are other muons produced within the MINOS ND. Note the curvature of the muon tracks due to the magnetic field within the MINOS ND. Image from [15].

During the majority of ArgoNeuT's run, the NuMI beam was operated in the low energy antineutrino mode; neutrino fluxes produced during this operation mode are described in [69]. The composition of the beam was $58 \%$ muon neutrino, $40 \%$ 
muon antineutrino, and $2 \%$ electron neutrino and antineutrino. The average energy for muon neutrinos was $9.6 \mathrm{GeV}$, and the average energy of muon antineutrinos was 3.6 GeV. The antineutrino mode run lasted 4.5 months with $1.25 \times 10^{20}$ protons on target (POT) acquired [15].

\subsection{Other LArTPC Neutrino Experiments at Fermilab}

Fermilab is the host to a number of LArTPC experiments in addition to ArgoNeuT. These experiments are being built to pursue many of the physics aims described in the last chapter, as well as providing experience for the future-communitycenterpiece DUNE LArTPC experiment. This section introduces the other Fermilabbased LArTPCs: MicroBooNE, SBND, ICARUS, LArIAT and DUNE.

3.4.1 MicroBooNE. MicroBooNE is the next LArTPC at Fermilab. At 89 tons, it is considerably larger than ArgoNeuT. The TPC is $2.56 \mathrm{~m}$ (drift) $\times 2.33 \mathrm{~m}$ (vertically) $\times 10.37 \mathrm{~m}$ (beam) . There are 3,456 collection plane wires oriented vertically and two induction planes with 2,400 wires each, oriented at \pm 60 degrees to vertical. MicroBooNE also has a light collection system composed of 32 photomultiplier tubes (PMTs) placed behind the wires. This system allows MicroBooNE to collect scintillation light produced as charged particles travel through the liquid argon. Scintillation light arrives at the PMTs almost immediately after it was emitted, giving MicroBooNE an immediate indicator of activity in the TPC. If there is no activity in the $\mathrm{TPC}$, the readout is not saved. In addition, this triggering diminishes the uncertainty in the location of particles in the drift direction, since the wire readout beings almost immediately after ionization occurs. MicroBooNE also features a third instrumented wire plane, which provides redundancy in case wires cease to function. Unlike ArgoNeuT, MicroBooNE sits in the Booster Neutrino Beam (BNB), a lower energy beam at Fermilab, at a distance of $470 \mathrm{~m}$ from the target [70]. MicroBooNE is on the surface, so cosmic rays will be a background. To help alleviate this, a cosmic 
ray tagger (CRT) was installed. The CRT, described in [71] is composed of panels which form four planes covering the detector on four sides. The panels are composed of scintillating strips placed side by side. Along the panels are wavelength shifting fibers which collect the light from the panels and send it to a Silicon Photomultiplier (SiPM). MicroBooNE began operation in 2015 and continues to this day.

The main physics goal of MicroBooNE is to clarify the MiniBooNE low-energy excess. This excess is a statistically significant surplus of electromagnetic events observed over a run of 10 years, in both neutrino and antineutrino mode running. This excess could be due to photons or electrons. If the former, then current modeling of certain nuclear process rates is incorrect, but this is a minor flaw. The latter indicates activity from electron neutrinos and antineutrinos, in excess of what is expected from the beam, indicating oscillations to electron (anti)neutrinos. This oscillation rate does not fit into the standard oscillation parameters between the three known neutrinos, but can fit into a model with the three known (also called "active") neutrinos and one sterile neutrino. This sterile neutrino would not interact via any force except for gravity and is not included in the Standard Model, so the confirmation of this excess would indicate physics beyond the Standard Model. Since MiniBooNE is a Cherenkov detector and is not able to distinguish between electrons and photons, it is unclear which particle produces this excess [28]. MicroBooNE, however, is a LArTPC, and the ability to distinguish between electrons and photons in such detectors has already been demonstrated [7].

3.4.2 SBND and ICARUS. Two more LArTPCs will join MicroBooNE on the BNB. The closest will be the Short Baseline Near Detector (SBND), a 112 ton TPC located $110 \mathrm{~m}$ from the BNB target. SBND's proximity to the target allows for high statistics measurements of neutrino-argon interactions as well as determining the unoscillated flux of the BNB. The other TPC is the refurbished ICARUS-T600, a 476 
ton detector. ICARUS (Imaging Cosmics And Rare Underground Signals) originally ran in the INFN-LNGS Gran Sasso laboratory in Italy and was exposed to the CNGS neutrino beam. The detector has since been moved to Fermilab and is placed $600 \mathrm{~m}$ from the target. Together, these three detectors make up the Fermilab SBN program. The main goal of the SBN program is to search for sterile neutrino oscillation [28].

While MicroBooNE will provide a measurement of the MiniBooNE excess, several uncertainties will remain. The other two detectors will help reduce these uncertainties. SBND will also provide more information about cross-sections and the beam profile, reducing two sources of uncertainties. In addition, the three detectors will allow for studies of both muon neutrino disappearance and electron neutrino appearance. The existence of a sterile neutrino can also be determined by a neutral current disappearance study. Since neutral current interactions do not depend on the flavor of the neutrino but do depend on whether the neutrino is active or sterile, this study provides another avenue [28].

3.4.3 LArIAT. LArIAT (Liquid Argon In A Testbeam) was the repurposed ArgoNeuT detector placed in a charged particle testbeam at Fermilab. It had several improvements over ArgoNeuT, such as an internal light collection system and better electronics. Using other detectors outside the TPC, LArIAT was used to study charged particle interactions in liquid argon in a testbeam of known particles and energies to calibrate and measure LArTPC response to different particle types. As such, it served as a research and development platform for future LAr experiments. Large LArTPCs currently being developed rely on measurements and experience from LArIAT's runs [67].

3.4.4 DUNE. The work done on all of the above experiments will also serve as input to another future experiment, the Deep Underground Neutrino Experiment (DUNE). DUNE is a long-baseline experiment with multiple detectors, three near 
detectors at Fermilab, and another, 40 kton LArTPC, in South Dakota. As its name implies, the far detector will be placed about one mile underground; this will help shield the detector from most cosmic rays. Reducing such backgrounds is important for any experiment, but especially so when the objects of study are rare and lowenergy events, such as proton decay and solar and supernova neutrinos. DUNE will also study neutrinos produced by a beam at Fermilab. DUNE will receive highenergy neutrinos from a new beam at Fermilab which will be similar in energy to the NuMI beam, but at a higher intensity. The near detectors will be able to measure the unoscillated neutrino flux as well as possibly study new physics, while the far detector will measure the oscillated flux, mass hierarchy and $\mathrm{CP}$ violation in addition to the physics studies mentioned earlier [3,27].

\subsection{Event Reconstruction in LArTPCs}

Event reconstruction in LArTPCs is performed in several steps, using the LArSoft reconstruction package [72]. In the first step, the unipolar and bipolar LArTPC wire signals (described in Sec. 3.1) undergo deconvolution based on a Fast Fourier Transform [15]. This process reduces noise and creates unipolar waveforms for all wire planes, shown as the blue lines in Figure 3.11.

Deconvolved wire signals are then processed through an automated "hit-finding" algorithm. To find a "hit" corresponding to deposited charge from drifting ionization electrons, wire waveforms are scanned for local maxima. If one is found and is above a predefined threshold, the peak signal is fitted with a Gaussian and is labeled as a "hit." From this Gaussian fit, information about the hit is extracted, for example the peak height and area of the Gaussian curve. The time sample number is also recorded as the location of the peak of the Gaussian. The number of electrons detected on the wire is is linearly proportional to the electronic signal recorded, in analog to digital conversion (ADC) units. An example of such channels or fitted 

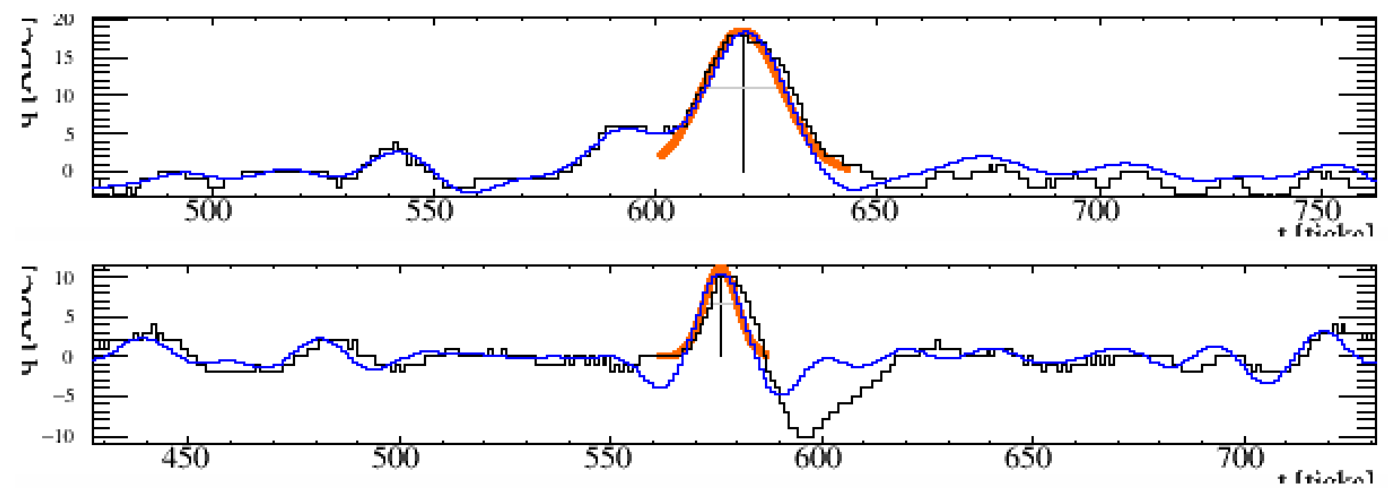

Figure 3.11. Wire signals from an ArgoNeuT event. Top plane is induction, bottom collection. The horizontal axis indicates time sample number, and the vertical axis indicates signal strength. Raw wire signals are in black (note the bipolar form of the raw signal in the induction plane), deconvoluted wire signals in blue, and fitted peaks in red.

hits is shown in the orange curves in Figure 3.11. Higher-level clustering of hits is then performed to generate reconstructed particle topologies, such as "tracks" and "showers" produced by ionization of heavy and light charged particles. The particle's kinematic and calorimetric quantities can then be reconstructed for use in physics analyses. The definition of a cluster used in this section is the same as that in the literature; the definition will be slightly different in later sections of this thesis. To form a cluster in data, a Density-Based Spatial Clustering of Applications with Noise (DBSCAN) algorithm [15] is used. The algorithm works by choosing an arbitrary hit and collecting other hits which are "density-reachable." A point $p$ is directly densityreachable to another point $q$ if it is within a specified distance and if $q$ is surrounded by sufficiently many points. A point $s$ is density reachable to $p$ if it is connected to $p$ by a series of directly density reachable points. Any points density reachable from any point in a cluster are part of the cluster. After clustering is completed, a Hough transform identifies line-like objects. The lines are then identified as tracks, shown in Figure 3.12.

Once tracks are identified, one can attempt to find a neutrino interaction ver- 


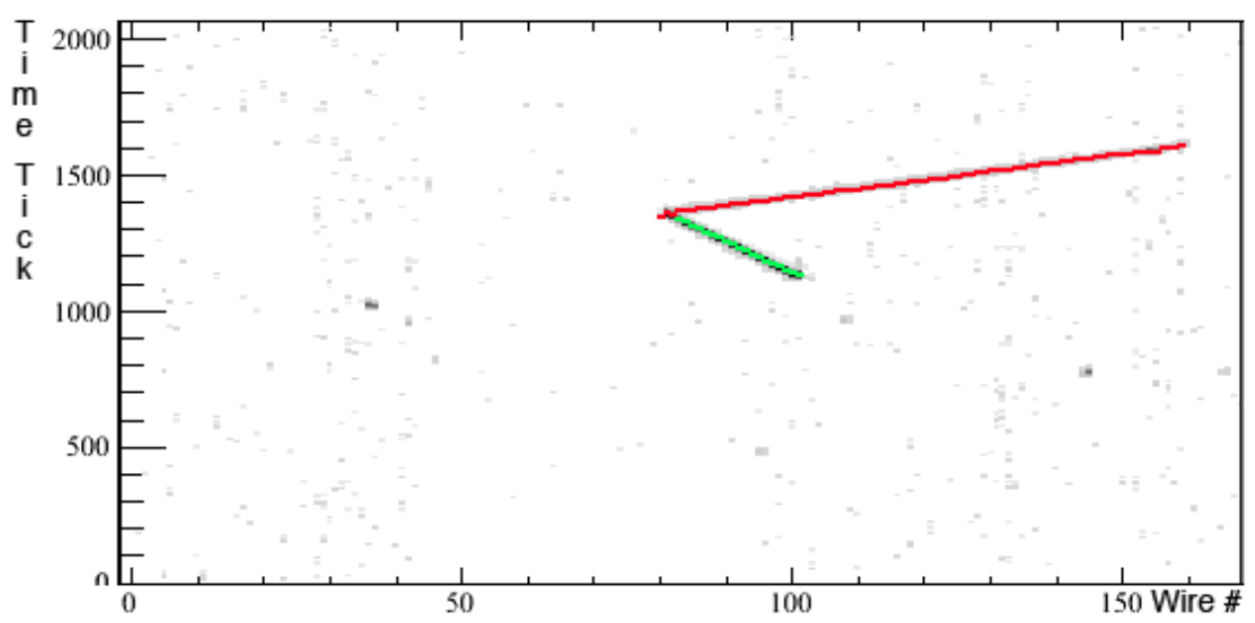

Figure 3.12. Tracks identified by the automated reconstruction in an ArgoNeuT neutrino event. Color indicates the two different tracks. Image from [15].

tex associated to the track(s). In experiments on the surface, the presence of cosmic rays makes identifying the neutrino interaction vertex difficult. Since ArgoNeuT was underground, with minimal cosmic ray interference, the process is simpler. For ArgoNeuT, the process begins by identifying either a track matched to another recorded track in MINOS ND (another nearby detector), or the longest track. The start of this track is chosen as the neutrino interaction vertex candidate. Other tracks can be associated with the vertex if their point of closest approach is within some limit. This process occurs in each wire plane; the two vertex points are then matched across planes for a 3D position.

LArTPCs have fine 3D position resolution, shown in Figure 3.13. By examining contemporaneous wire activity across planes, it is possible to reconstruct the 2D trajectory of a charged particle. To obtain the third coordinate, i.e. along the drift direction, timing information is used. Since the drift velocity is constant across the drift direction, it is possible to find the location of the ionization by examining when the ionization was detected by the wires. In older TPCs such as ArgoNeuT, the time is defined with respect to the arrival of the beam. To increase the accuracy of the drift 
coordinate, LArTPCs are now outfitted with light collection systems, typically PMTs which detect scintillation light. In addition to ionizing electrons as a particle travels in the detector, scintillation light is also produced. The scintillation light signal is used to provide an absolute timestamp on the wire readout. In this case, there is no bias in the drift time.

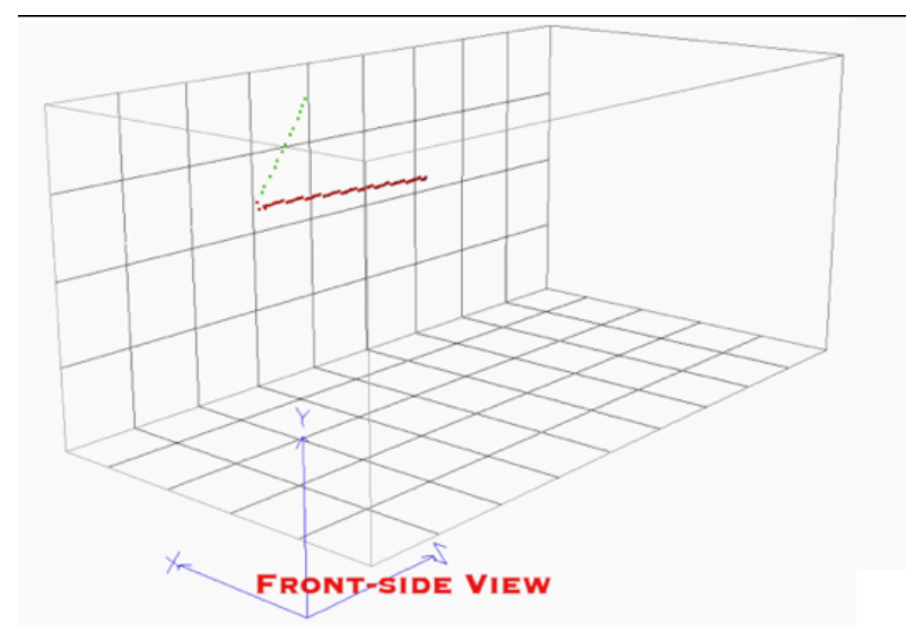

Figure 3.13. The same event as in Figure 3.12, reconstructed in 3D. Note that the tracks exit the detector. Image from [15].

The number of electrons collected in a LArTPC is not equal to the number of electrons ionized by a charged particle and this must be accounted for during reconstruction. This is due to two factors, electron recombination and the presence of impurities in the liquid argon. Recombination occurs when ionized electrons attach to nearby argon ions. This effect depends on the electric field in the TPC, the $d E / d x$ of the particle causing the ionization and the angle of the particle's trajectory relative to the electric field. As the electric field increases, the amount of charge remaining, called the recombination factor, decreases. It is of course impossible to increase the electric field indefinitely, so there will always be some loss in the charge ionized. As shown in Figure 3.14, the recombination factor decreases as $d E / d x$ increases. There are two main models for recombination, the Birks model and the Box model [16]. The Birks model was developed to determine the loss of scintillation light and performs fairly 
well in modeling loss of ionized electrons. It is however limited in its range of $d E / d x$, and applying it is difficult for high $d E / d x$ values. The generic Box model does not work well at low $d E / d x$ values, but with small modifications to certain constants in the model, the new modified Box model adequately describes recombination. LArTPC experiments use this modified Box model, described in [16] and shown in Eq. 3.7,

$$
\frac{d E}{d x}=\frac{\exp \left[\beta W_{\text {ion }}(d Q / d x)\right]-\alpha}{\beta}
$$

where $\beta=0.30 \mathrm{~cm} / \mathrm{MeV}, W_{\text {ion }}=23.6 \mathrm{eV}$ is the ionization energy of argon, $d Q / d x$ is the amount of charge ionized per unit length and $\alpha=0.93$ is a constant. A study of electron recombination using ArgoNeuT is found in [16].

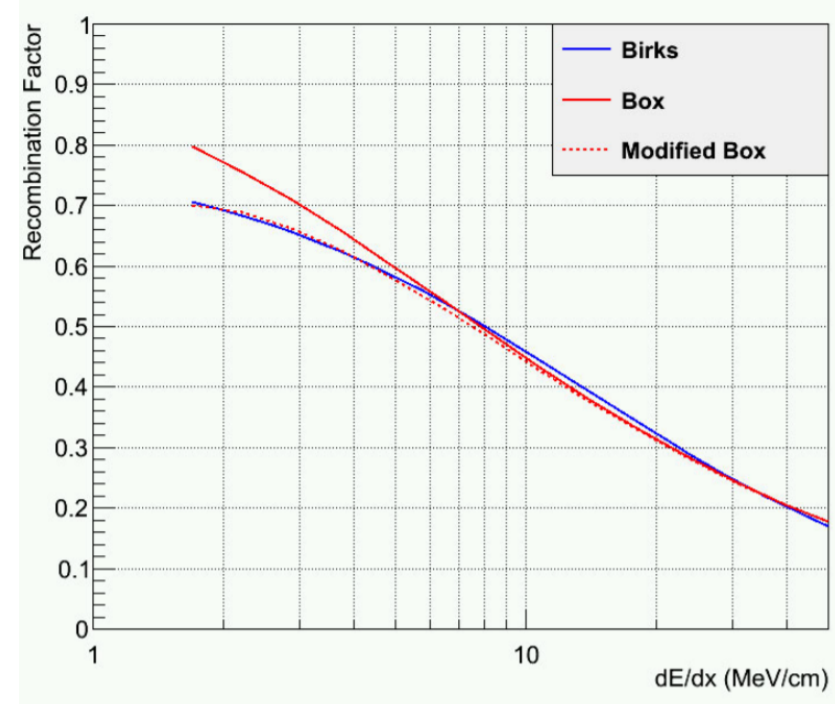

Figure 3.14. Recombination factor as a function of $d E / d x$ in an electric field of 480 $\mathrm{V} / \mathrm{cm}$ for a variety of models. ArgoNeuT and other LArTPCs currently use the Modified Box Model. Image from [16].

The other factor impacting the amount of charge collected is the presence of impurities in the liquid argon. Since argon is a noble element, free electrons will not attach to it, but they may attach to elements that are electro-negative, such as oxygen. The number of electrons captured on impurities increases exponentially with time, so electrons ionized closer to the cathode are more likely to be captured than 
those ionized close to the wire planes. This effect is called the electron lifetime and can be measured either with specialized equipment or by examining the signal loss of muon tracks which pierce the anode and cathode. In reconstruction, the signal is amplified with the following relation

$$
Q_{\text {ion }}=Q_{\text {coll }} \exp [t / \tau]
$$

where $Q_{\text {ion }}$ is the amount of charge ionized, $Q_{\text {coll }}$ is the amount of charge collected, $t$ is the drift time, and $\tau$ is the measured electron lifetime. It is possible to remove impurities in argon, and improvements in the removal process have largely mitigated this problem.

LArTPCs are able to identify particles by examining the energy deposited per unit length $(d E / d x)$, which depends on the particle and its momentum or energy. A more massive particle will deposit more energy per unit length than a lighter one. In addition, as a particle comes to a stop, the amount of energy deposited increases. A combination of these two properties allows one to clearly identify an electron, proton or kaon track. Determining whether a track was due to a pion versus a muon is more difficult as they have similar $d E / d x$ values. A plot of $d E / d x$ as a function of the residual range of various particles is give in Figure 3.15. Further information about the passage of particles through matter is found in Sect. 3.2. 


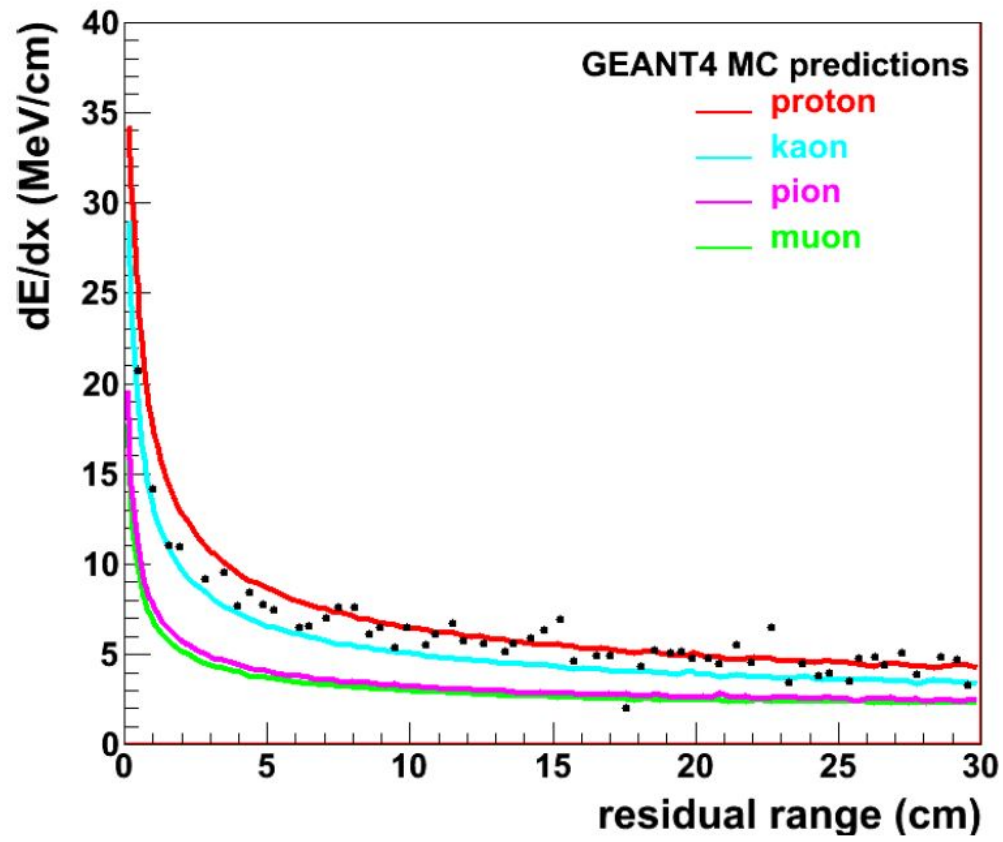

Figure 3.15. Amount of energy deposited per unit length $(d E / d x)$ versus residual range for a variety of particles in liquid argon. Image from [15]. 


\section{CHAPTER 4 \\ LARTPC PHYSICS STUDIES}

LArTPCs have many features which make them ideal detectors for neutrino and BSM experiments. Their high resolution imaging combined with calorimetry allow for a large variety of physics studies. This chapter focuses on a range of physics studies which can be performed with a LArTPC, starting at the GeV scale and moving to the $\mathrm{MeV}$ scale.

\subsection{Neutrino Physics Studies at GeV Scales}

The majority of physics studies in LArTPCs have been performed at the $\mathrm{GeV}$ scale, using neutrinos from accelerators. Among the most common are cross-section measurements and other neutrino interaction measurements. Precise determination of cross-sections is important for determining interaction rates and reducing systematic uncertainties, especially in oscillation experiments. Cross-sections on argon have been measured in ArgoNeuT [17,69,73,74] and MicroBooNE [75,76].

Oscillation measurements make use of clearly defined topologies of beam neutrino interactions. Oscillation studies have been performed in ICARUS [77]. The major goal of MicroBooNE is to understand the MiniBooNE "Low Energy Excess," essentially an oscillation study. The goal of searching for sterile neutrino oscillation is shared by the SBN program as a whole [28].

Since the neutrinos are high-energy, events appear with clearly defined topologies. For example, Figure 4.1 shows an electron neutrino $\mathrm{CC}$ interaction event in ArgoNeuT, where the electron shower is clearly visible along with several tracks at the vertex. Figure 4.2 shows a muon neutrino $\mathrm{CC}$ event, also in ArgoNeuT, with 
a long muon track (in green) and two, short proton tracks. Note that the protons leave the vertex back-to-back. This is due to nuclear effects within the target nucleus, another topic which can be studied in LArTPCs [17].

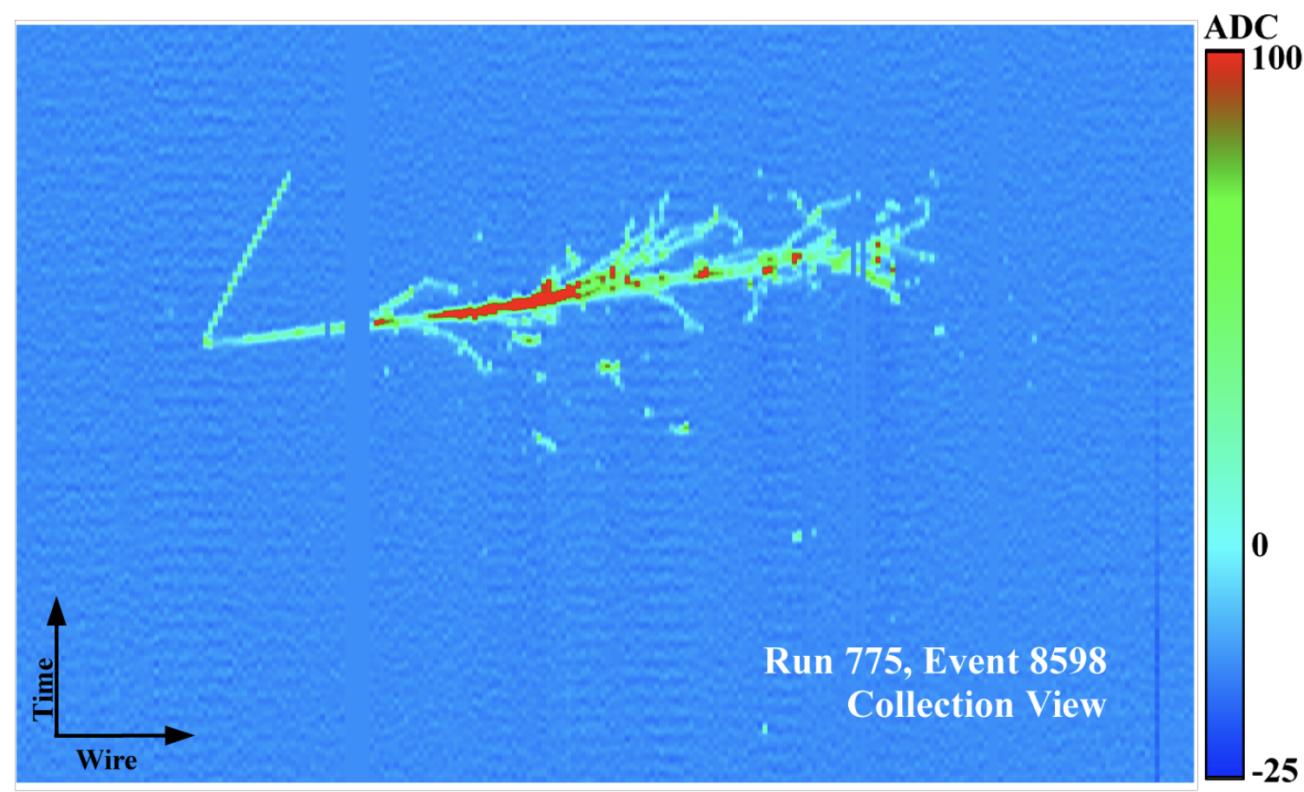

Figure 4.1. An electron neutrino CC event in ArgoNeuT displaying a high-energy electron, seen as a shower. Other hadronic activity is seen at the vertex. Only the collection plane is shown Wire number is indicated on the x-axis. The y-axis indicates time in ticks. Color indicates amount of charge collected. Image from [7].

\subsection{Benefits of MeV-Scale Abilities for GeV-Scale Physics Studies}

Current LArTPC experiments have difficulty accounting for the amount of energy lost to neutrons in a neutrino interaction. The problem is even worse in antineutrino interactions, where the nucleon with the most energy is a neutron. Figure 4.3 shows predicted energies of neutrons emitted in antineutrino interactions on polystyrene in MINERvA. The antineutrinos have an average energy of $3 \mathrm{GeV}$, typical of beam neutrinos. The predictions come from three neutrino interaction generators and show significant disagreement, yet all agree that the amount of energy taken away by the neutron can be quite high, approaching $10 \%$ of the neutrino's energy [78]. Since neutrons are neutral, it is impossible to see them directly. The only way to detect 


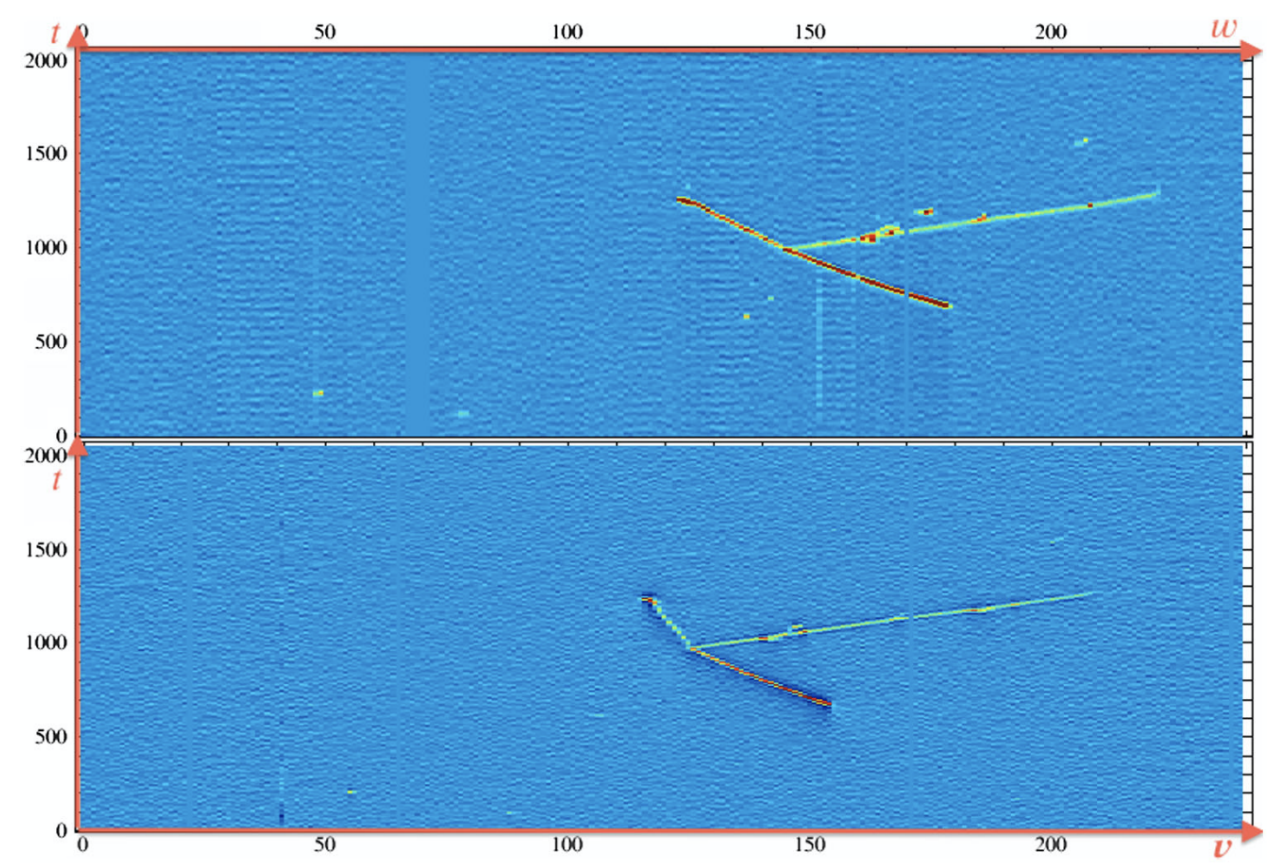

Figure 4.2. An muon neutrino CC event in ArgoNeuT. The muon is seen as the long, nearly horizontal track. This event display also shows two protons traveling away from the vertex back-to-back, indicating nuclear effects. Top plane is collection, bottom induction. Wire number is indicated on the x-axis. The y-axis indicates time in ticks. Color indicates amount of charge collected. Image from [17].

them is after secondary interactions, primarily by electrons from Compton scatters of photons from inelastic scattering of neutrons, as described in Sec. 3.2.3. The energies of these electrons are typically less than $3 \mathrm{MeV}$, so MeV-scale reconstruction methods are crucial for reconstructing neutrons to determine their energies and multiplicities.

In electron (anti)neutrino CC events and events where a high energy photon is found (such as $\pi^{0}$ decays), one or more showers is reconstructed in the event. An example of such a shower produced by a high-energy electron is shown in Figure 4.1. Showers are caused by high-energy electrons, and high-energy electrons lose a portion of their energy in the form of radiative photons. The higher the energy of the electron, the more energy is lost in radiative photons. These photons are typically low energy and deposit their energy by Compton scattering, producing lower energy electrons away from the core of the shower. MicroBooNE has studied this effect and has shown 


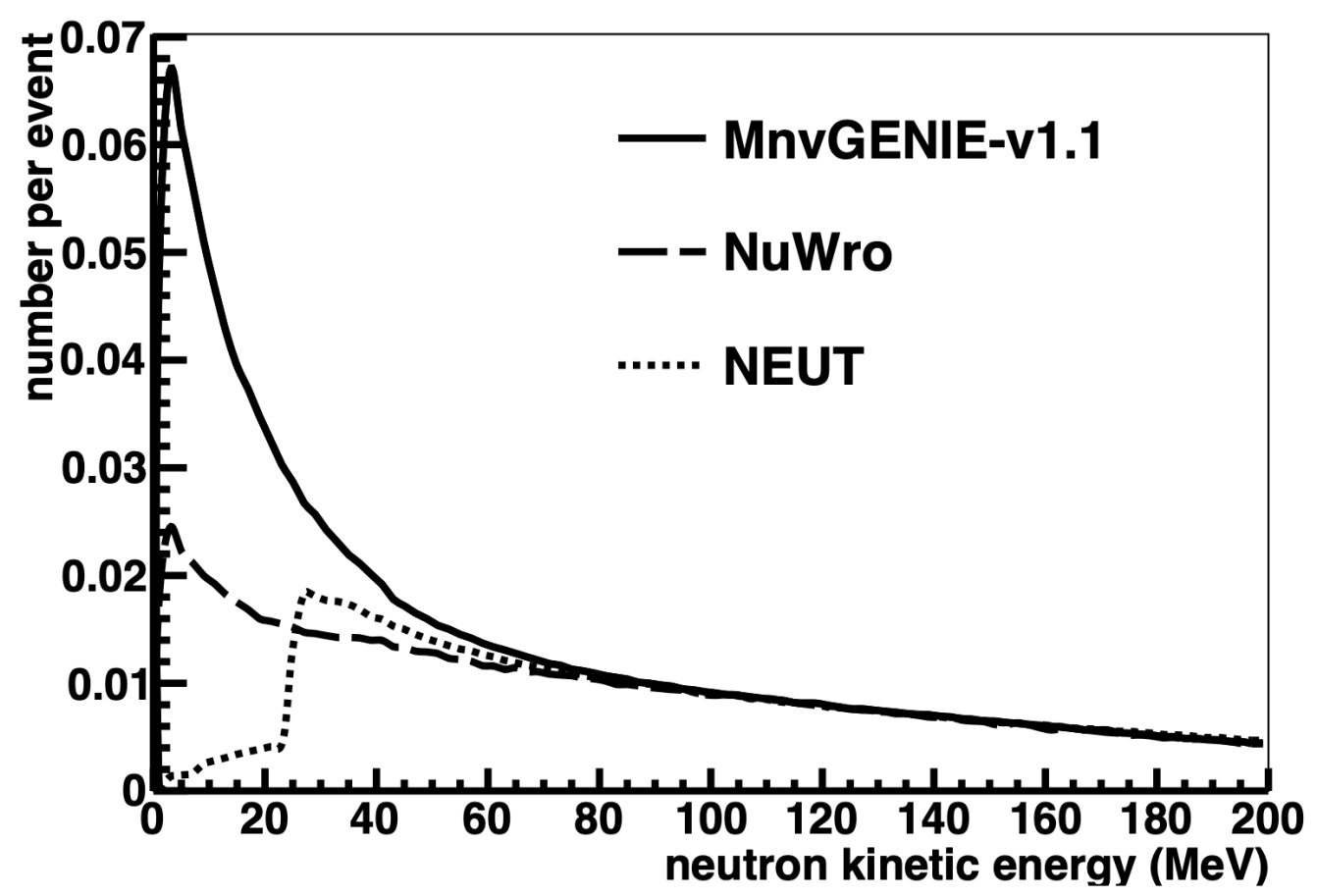

Figure 4.3. Energy spectrum of neutrons as predicted by three neutrino interaction generators for $3 \mathrm{GeV}$ antineutrinos interacting on polystyrene. This energy is typical of beam neutrinos. While there is disagreement between the generators, all three indicate that a substantial portion of the antineutrino's energy can be given to neutrons. Image from [18].

that a substantial portion of the electron's energy can be lost to these photons [12], as shown in Figure 4.4, which compares the true spectrum of Michel electrons (red) from cosmic rays to the reconstructed spectrum using only energy lost due to ionization and not radiative photons (gray). The difference in the spectra shows that the amount of energy lost to photons is large. The ability to reconstruct the Compton scatters of these photons would help bring the reconstructed spectrum closer to the true spectrum, for both Michel electrons and higher-energy electrons.

After a GeV-scale neutrino interaction, the final-state nucleus is often left in an excited state. The nucleus deexcites by releasing photons, the energies and multiplicities of which depend on the specific isotope of the final state nucleus. While 


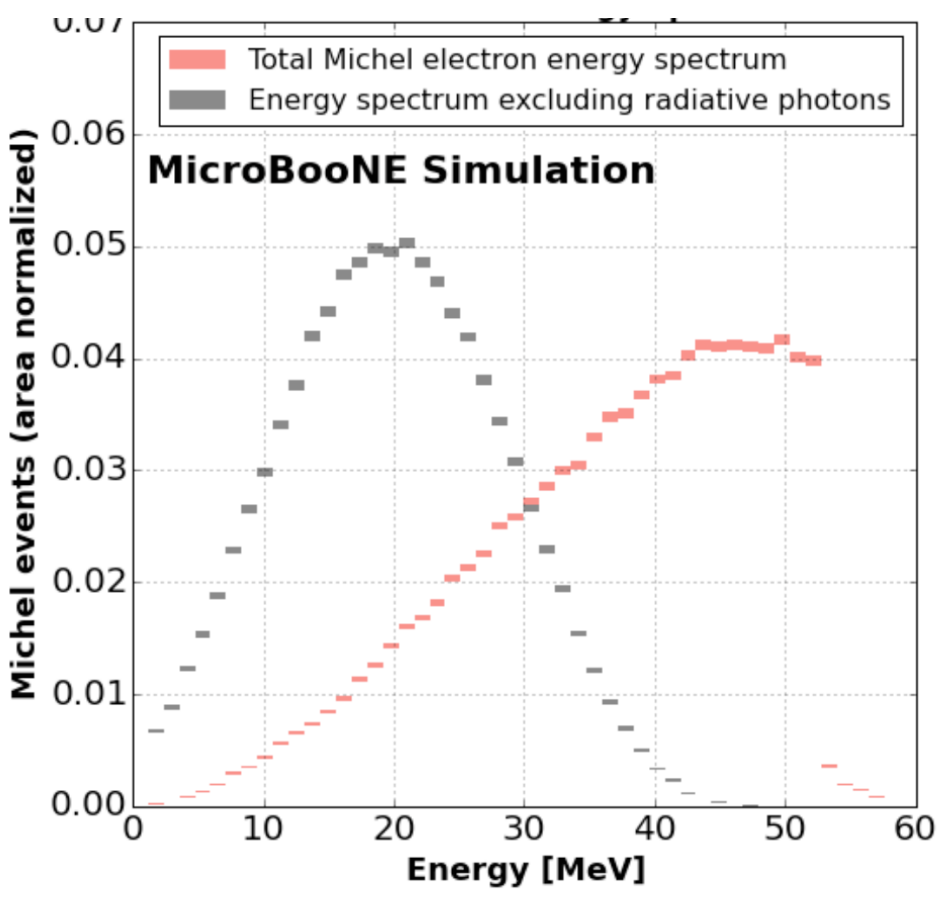

Figure 4.4. Energy spectrum of Michel electrons from a simulation of cosmic rays in MicroBooNE. Red denotes the true Michel electron spectrum. Black denotes the reconstructed spectrum using only energy deposited by ionization; this excludes energy lost in the form of radiative photons. Distributions are area normalized. From [12].

the energy released in these photons is relatively small $(<10 \mathrm{MeV}$ typically), these photons can provide important information. For one, they will allow for a more complete reconstruction of the neutrino's energy. In both cross-sections and oscillation studies, knowledge of the neutrino's energy is essential. The ability to accurately reconstruct low-energy activity demonstrated in this thesis will allow experiments to more accurately account for the neutrino's energy [28]. The photons may also enable identification of the final-state nucleus via gamma spectroscopy, which could indicate whether a neutrino or antineutrino interacted or what final-state particles should be present at an interaction vertex.

\subsection{Neutrino Physics Studies at MeV Scales}

In addition to the studies mentioned, future LArTPC experiments such as the 
SBN program and DUNE will have an even broader realm of physics studies, many focusing on MeV scales. Some examples are described below. A major goal of DUNE is to detect and reconstruct supernova neutrinos should an event occur during the experiment's lifetime. This would provide insight into the processes occurring inside a supernova. As discussed in Section 2.3, supernova neutrinos have energies $<60$ $\mathrm{MeV}$. These neutrinos are not energetic enough to produce muons or tau leptons, but they can produce electrons in CC absorption and scattering events. In CC absorption events,

$$
\nu_{e}+{ }^{40} \mathrm{Ar} \rightarrow e^{-}+{ }^{40} \mathrm{~K}^{*}
$$

and

$$
\bar{\nu}_{e}+{ }^{40} \mathrm{Ar} \rightarrow e^{+}+{ }^{40} \mathrm{Cl}^{*}
$$

the electron neutrino (antineutrino) is absorbed and an electron (positron) is emitted. The nucleus is also left in an excited state (denoted by the ${ }^{*}$ ). To deexcite, the nucleus releases photons. Since the energies of the neutrinos are low, these deexcitation photons account for a large fraction of the neutrino's energy. In scattering events,

$$
\nu_{x}+e^{-} \rightarrow \nu_{x}+e^{-}
$$

any flavor of neutrino can scatter with an electron. The nucleus is left in the ground state, so no photons are emitted. Table 4.1 lists expected event rates for the different types of interactions in DUNE of neutrinos from a supernova $10 \mathrm{kpc}$ away. To perform oscillation measurements, it is necessary to be able to identify which interactions are due to electron (anti)neutrinos, which requires looking for absorption events. A way to distinguish between an absorption event and a scattering event is by the presence of deexcitation photons. It is therefore crucial to be able to reconstruct such photon activity which exists at the $\mathrm{MeV}$-scale. An example of such activity can be seen in Figure 4.5, which depicts a simulated supernova electron neutrino absorption event in the ArgoNeuT detector. The main electron emitted is visible as the relatively 
long trail. Near the electron are small blips due to Compton scatters of deexcitaiton photons which indicate this is an absorption reaction. Until the work presented in Chapter 5, analysis methods could only reconstruct the main electron; activity from the deexcitation photons was neglected [10,27].

Table 4.1. Expected interaction rates in DUNE of neutrinos from a supernova 10 kpc away. Livermore model does not assume oscillations, but GKVM assumes collective oscillations. From [3].

\begin{tabular}{ccc}
\hline \hline Channel & Events "Livermore" Model & Events "GKVM" Model \\
\hline$\nu_{e}+{ }^{40} \mathrm{Ar} \rightarrow e^{-}+{ }^{40} \mathrm{~K}^{*}$ & 2720 & 3350 \\
$\bar{\nu}_{e}+{ }^{40} \mathrm{Ar} \rightarrow e^{+}+{ }^{40} \mathrm{Cl}^{*}$ & 230 & 160 \\
$\nu_{x}+e^{-} \rightarrow \nu_{x}+e^{-}$ & 350 & 260 \\
Total & 3300 & 3770 \\
\hline
\end{tabular}

Another interesting example is solar neutrinos. The sun produces even lowerenergy neutrinos as described in Sec. 2.3. Detection in a LArTPC is again by electron neutrino absorption and scattering. While the energy requirement for the former is $5 \mathrm{MeV}$, excluding much of the solar neutrino flux, studies can still be performed. For example, DUNE would be able to measure the ${ }^{8} \mathrm{~B}$ and hep flux, the latter of which has never been measured [79]. Since the sun produces only electron neutrinos, oscillation measurements can be performed, allowing for more precise fits of $\theta_{12}$ and $\Delta m_{12}^{2}$. DUNE's capability to improve these two parameters is seen in Figure 4.6, which shows present measurements on the left and the potential of DUNE's measurements on the right. The curves indicate 1,2 and $3 \sigma$ confidence levels. To perform such a measurement it is necessary to disentangle $\mathrm{CC}$ electron neutrino absorption (Eq. 4.1) from scattering (Eq. 4.3), as discussed above. It should be noted that this Figure separates these two interactions by using an angular cut. With the ability 


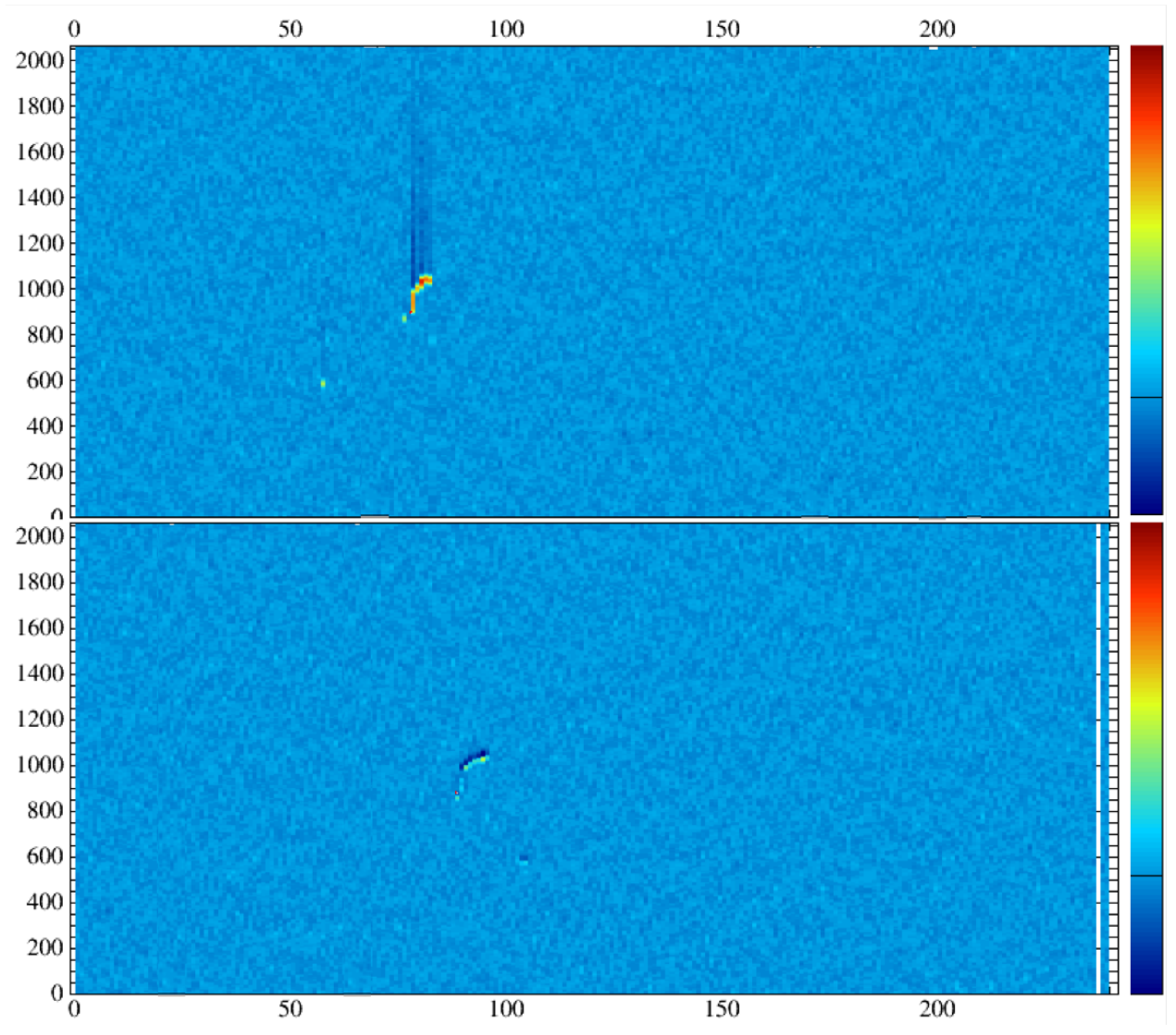

Figure 4.5. A simulated supernova neutrino CC absorption event in ArgoNeuT. A short electron track is seen along with activity due to deexcitation photons. Top plane is collection, bottom induction. Wire number is indicated on the x-axis. The $\mathrm{y}$-axis indicates time in ticks. Color indicates amount of charge collected.

to reconstruct deexcitation photons, separating the interactions is easier, and results should be enhanced [19].

Accelerators also produce low energy neutrinos from the decay at rest of muons, pions and kaons [80]. These neutrinos are similar in energy to supernova neutrinos. The energies and flavors are also well known, so oscillation studies can be performed using these neutrinos. Such oscillations would occur at shorter distances (since the energy is lower) compared to typical accelerator neutrinos, so detectors can be placed closer to the source, instead of hundreds of kilometers away as is typical 

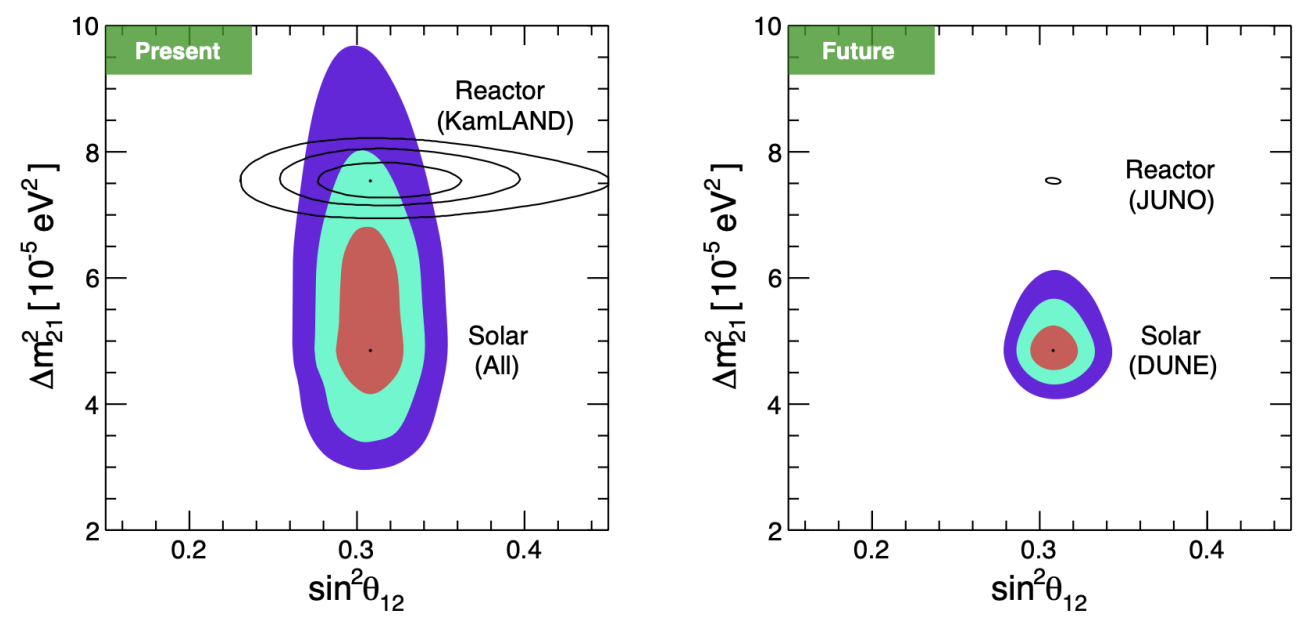

Figure 4.6. Present (left) and future (right) measurements of neutrino mixing parameters with solar and reactor neutrinos. Curves indicate 1, 2 and $3 \sigma$ confidence intervals. DUNE will be able to significantly improve these values by reconstructing solar neutrinos. From [19].

for accelerator experiments.

\subsection{BSM Physics Studies and MeV-Scale Capabilities}

LArTPCs are versatile detectors, able to perform a variety of BSM physics studies. Some of these studies can be performed with a neutrino beam. This section some examples of possible studies that can benefit from MeV-scale LArTPC reconstruction. A more complete discussion is found in [81].

As described previously, the signal for millicharged particles is isolated lowenergy depositions, pointing back to the production point. As a result, MeV-scale reconstruction methods are crucial for performing a search for these theoretical particles in LArTPCs. The demonstrated ability of LArTPCs to perform reconstruction at the MeV-scale allowed ArgoNeuT to perform the first search for millicharged particles in a LArTPC (Chapter 6).

Due to their high resolution, LArTPCs can study neutrino tridents. A trident is a process where a neutrino interaction produces a neutrino and two leptons with 
opposite signs. While this is allowed in the SM, they are very rare and depending on the angle between the two leptons, could indicate new physics. Other tridents, such as $\pi^{+} l^{-}$, where $l$ is a lepton are also theoretically possible. There are two unresolved questions for reconstruction of tridents in LArTPCs. The first is what is the smallest angle between the two charged particles such that they can be distinguished. The second is how well a LArTPC can determine which particles make up the trident. Distinguishing between charged pions and muons is difficult in LArTPCs, and sign determination is difficult as well.

Tridents can arise from the decay of heavy sterile neutrinos. This activity is accompanied by the presence of activity from deexcitation photons, namely Compton scatters, however the photon activity will be separated from the trident activity. While the trident activity is likely to have energies in the GeV-range, the photons are likely to have energies of a few $\mathrm{MeV}$. These heavy neutrinos can arise from meson decay and mixing of active neutrinos and may play a role in the generation of neutrino mass. Since they are more massive than the active neutrinos, they will arrive in the detector later, so LArTPCs can search for them by examining activity after the expected arrival of active neutrinos [81].

In addition to running the accelerator in neutrino production mode, the SBN program will also have the ability to study sub-GeV dark matter by operating when the accelerator runs in "beam-dump" mode, where the proton beam misses the target and instead hits the absorber, thereby minimizing the neutrino flux. 


\section{CHAPTER 5}

\section{FIRST DEMONSTRATION OF MEV-SCALE PHYSICS IN LIQUID ARGON TIME PROJECTION CHAMBERS USING ARGONEUT}

LArTPCs have already demonstrated excellent reconstruction capabilities at GeV-scale energies, but their capabilities at the MeV-scale are unknown. As earlier discussed, numerous GeV-scale studies would benefit from $\mathrm{MeV}$-scale reconstruction abilities. Futhermore, several other low-energy neutrino and BSM physics studies depend on the ability to detect and reconstruct activity at the $\mathrm{MeV}$-scale and below. This thesis seeks to demonstrate these capabilities and perform a BSM analysis using such activity.

The following is a study in ArgoNeuT on reconstructing low-energy $(<3 \mathrm{MeV})$ depositions from de-excitation photons and inelastic neutron scattering from neutrino interactions. The analysis is published in Physical Review D [82] and was presented at a Fermilab Joint Experimental-Theoretical Physics seminar. The work, based entirely on my own analysis efforts but building on low-level tools developed by the ArgoNeuT collaboration, has been well-received, with 13 citations as of the writing of this thesis and a follow-up analysis being performed in MicroBooNE. Authors have written on the impact of this work for future LArTPC experiments [78].

\subsection{MeV Scales in LArTPCs}

Identifying and reconstructing particles at $\mathrm{MeV}$ energies in a LArTPC presents challenges not present at higher energies. At higher energies (> $100 \mathrm{MeV})$, charged particles will travel far in liquid argon, a distance of several centimeters to meters. They will leave detectable signals on dozens or hundreds of wires that can be used to reconstructing the identity and energy of the particle (see Sec. 3.5). However, charged 
particles with kinetic energies of a few $\mathrm{MeV}$ or less may travel a distance shorter than the distance between adjacent wires in LArTPCs $(3-5 \mathrm{~mm})$. As a result, current analysis methods which use many wire signals to reconstruct the physics occurring in the LArTPC are ineffective at these energies, and new, low-energy-specific methods are needed.

To accomplish these goals, we used data acquired from ArgoNeuT and searched for small energy depositions associated with neutrino events. We then compared them to predictions from the FLUKA neutrino interaction generator [83]. With the new topological reconstruction tools we developed, we found clear evidence of activity due to deexcitation of the final-state nucleus and inelastic scattering of neutrons in the analyzed events.

\subsection{Production and Interaction of Low-Energy Photons in Neutrino-Argon Interactions}

MeV-energy photons are produced in two possible ways in neutrino-argon interactions, by deexcitation of the final-state nucleus and by inelastic scattering of final-state neutrons in the argon. When a neutrino interacts with an ${ }^{40} \mathrm{Ar}$ nucleus, that nucleus is often left in an excited state. If the energy of the excited nucleus is high enough, deexcitation of the nucleus is accomplished first by releasing nucleons. When the energy is too low to release nucleons, the nucleus deexcites by releasing photons. The final-state nucleus is then typically an isotope of argon, potassium or chlorine. These excited nuclei will produce 1-6 photons with energies ranging from 0.1 $\mathrm{MeV}-10 \mathrm{MeV}$. Final-state neutrons which inelastically scatter off an ${ }^{40} \mathrm{Ar}$ nucleus will also produce photons in the energy range of interest as the nucleus de-excites [84].

Photons are neutral particles and cannot be detected directly. It is possible to detect them after they undergo an interaction as described in Section 3.2.2, by detecting electrons. Since the most likely interaction process for $\mathrm{MeV}$-energy photons 
is Compton scattering, and a photon can Compton scatter multiple times, we are left with multiple topologically isolated energy depositions inside the LArTPC. Higher energy photons can also interact via pair-production, but this is a subdominant interaction for the energies of the photons considered here. The scale of the distance between subsequent energy depositions for one photon is given by the radiation length $\left(X_{0}\right)$, which in liquid argon is $14 \mathrm{~cm}$.

FLUKA [83] is the only neutrino MC interaction generator that includes the simulation of both mechanisms of low-energy photon production in GeV-scale neutrino interactions. Figure 5.1 shows the energies and numbers of photons from charged current interactions of muon neutrinos from the NuMI beam interacting and depositing energy in a volume of liquid argon with the dimensions of ArgoNeuT, according to a FLUKA simulation (see Section 5.3 for details). A significant overlap in both the energies and numbers of photons from the two processes is visible, making separation of the source of energy depositions difficult based on these metrics alone. Since ArgoNeuT is a small detector, a significant amount of in-TPC generated photons could deposit non-trivial amounts of energy outside of the TPC, making it difficult to completely reconstruct a photon's energy. It is also notable that $24 \%$ of product nuclei in this simulation are found in the ground state and produce no photons.

The typical signature of low energy photon-produced electrons is expected to be seen as isolated energy depositions (blips) around the neutrino interaction vertex. An example can be seen in Figure 5.2, where a typical ArgoNeuT neutrino event is shown.

\subsection{Datasets}

This analysis uses two primary real datasets from ArgoNeuT's antineutrino 

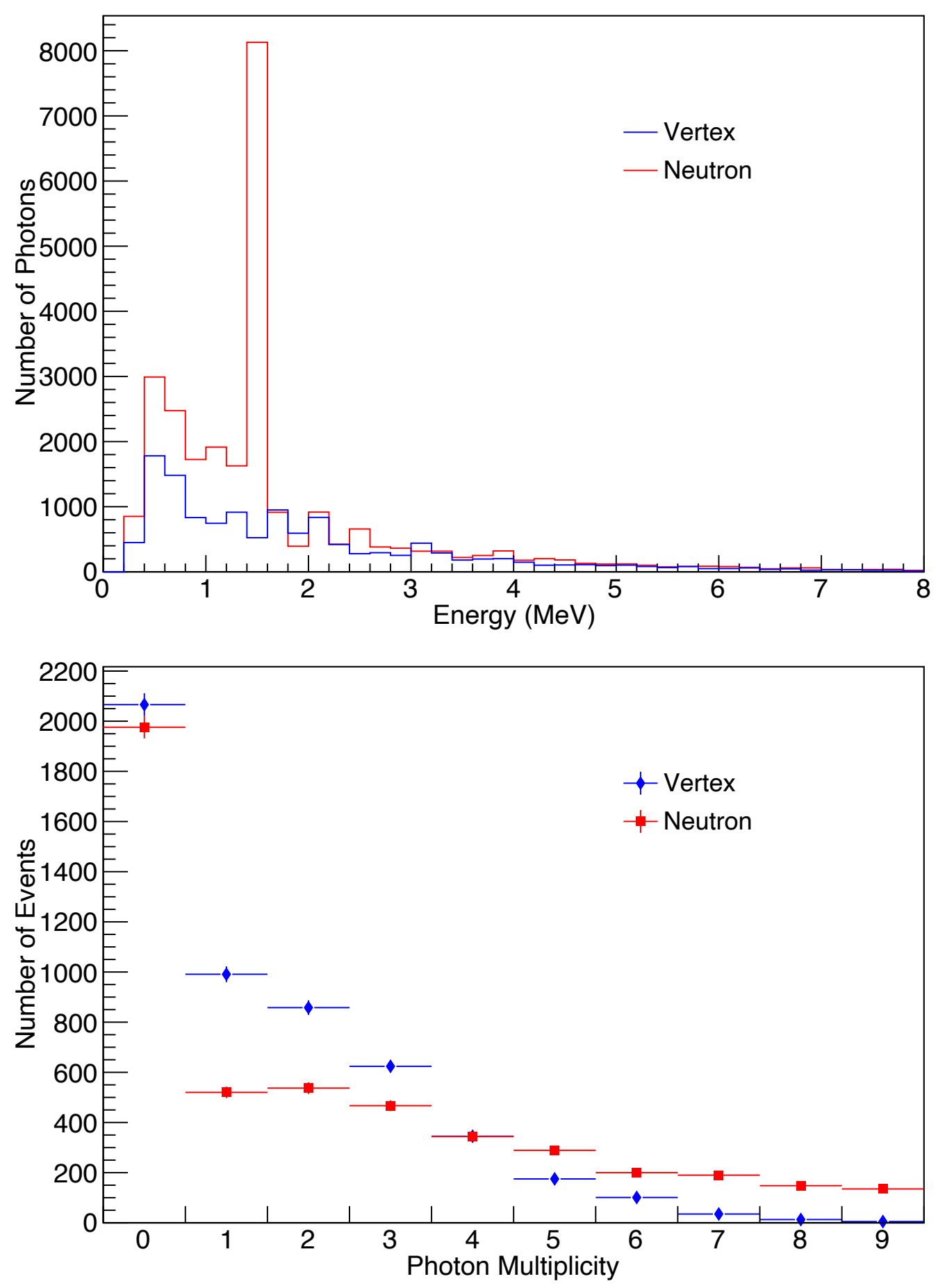

Figure 5.1. Energy (top) and multiplicity (bottom) of low-energy photons from charged current interactions of muon neutrinos from the NuMI beam interacting and depositing energy in a volume of liquid argon with the dimensions of ArgoNeuT. Color indicates source of photon (red are de-excitation photons, blue are photons produced by neutrons). For a photon to be tracked in the simulation, it must have an energy $\geq 0.2 \mathrm{MeV}$. 


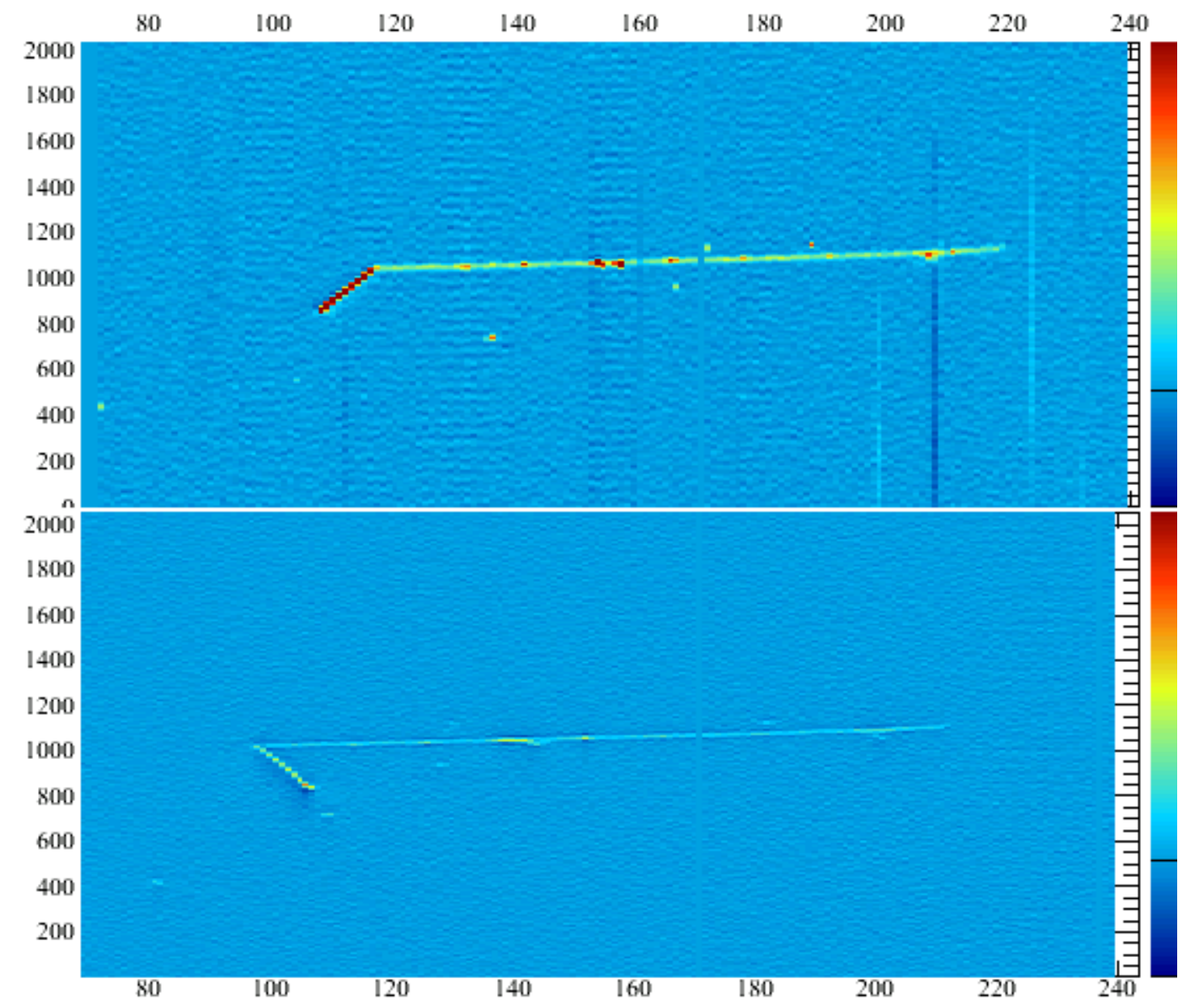

Figure 5.2. A neutrino event (raw data) with one track reconstructed as a muon and one track reconstructed as a proton. Possible gamma activity (isolated blips) is visible around the muon track. Top plane is collection, bottom induction. Wire number is indicated on the x-axis. The y-axis indicates time in ticks. Color indicates amount of charge collected. 
mode run which includes over 4 million data triggers. Neutrino events with simple, low track multiplicity final-state topology have been selected for the present analysis, as more complex events make the selection of isolated low-energy signatures more difficult. The first dataset, termed the neutrino dataset, is a subsample of muon neutrino and antineutrino events from the ArgoNeuT Charged Current pion-less (CC $0 \pi$ ) events sample, i.e. muon (anti)neutrino charged current events that do not produce pions in the final state. The selection and analysis of these events, reported in [85], requires matching to a MINOS ND (anti)muon track, and any number of proton tracks in the final state $(\mu+N p$ events). In none of the events is there a reconstructed electromagnetic shower or a track identified as a charged pion. Threshold for proton (pion) identification is 21 (10) MeV. From the CC 0-pion sample we have selected a subsample of events with one muon and up to one proton in the final state (CC $0 \pi$, 0 or 1 proton events) for the present analysis. There are 552 such events.

The second dataset, termed the background dataset, was obtained by examining triggers immediately before and after each selected neutrino event trigger. These triggers are "empty events" and do not appear to contain a neutrino interaction but do contain ambient gamma ray backgrounds, intrinsic ${ }^{39} \mathrm{Ar}$ activity, above threshold electronics noise, and products (neutrons and photons) of upstream neutrino interactions. These features are also present in the neutrino events previously described, so the background dataset is used for a data-driven modeling of the background in the selected neutrino events. A total of 1970 background events were considered.

We compare the data with a MC dataset, generated using the FLUKA MC neutrino interaction generator. We produced simulated neutrino interactions in ArgoNeuT using FLUKA and the energy spectrum of the NuMI beam. A simplified ArgoNeuT detector geometry was inserted into FLUKA. In addition to producing all the final-state particles emerging from the neutrino interaction, including hadron 
re-interaction inside the nucleus (nuclear effects), FLUKA also simulates the physics of the final-state nucleus, resulting in the production of final-state de-excitation photons. FLUKA was also used to propagate final-state neutrons inside the LAr volume, resulting in the simulation of energies and locations of secondary neutron-produced photons. The FLUKA-determined properties of non-neutron final-state particles and secondary neutron-produced photons were then used as input to a LArSoft [72] MC simulation of ArgoNeuT and propagated through the detector simulation, signal processing, and reconstruction stages as for real data. CC $0 \pi 0,1$ proton events, i.e. events with one muon track entering the MINOS ND and up to one additional proton with kinetic energy $>21 \mathrm{MeV}$ and no pions with kinetic energy in $>10 \mathrm{MeV}$ in the final state, compose the selected MC samples for the present analysis. Electronics noise and ambient radioactivity were not simulated. Instead, the background dataset described above was used to directly overlay these contributions on top of the MC dataset.

\subsection{Event Reconstruction}

As discussed in Section 5.2, the radiation length in liquid argon is $\sim 14 \mathrm{~cm}$, and $\mathrm{MeV}$ photon-produced electrons have ranges of a millimeter to a centimeter, as shown in Figure 5.3. Consequently, for the present analysis a signal on the wire planes consists of very short clusters of hits on consecutive wires on both active planes of the TPC, topologically isolated from the rest of the event's features, with some possible degree of concentration around the interaction vertex, as shown in Figure 5.2.

The same reconstruction procedure has been applied to all the real and MC selected data sets described in the previous Section. The reconstruction proceeded through two steps, one "standard" reconstruction step, followed by a low-energy specific second step, described in Section 5.4.1. 


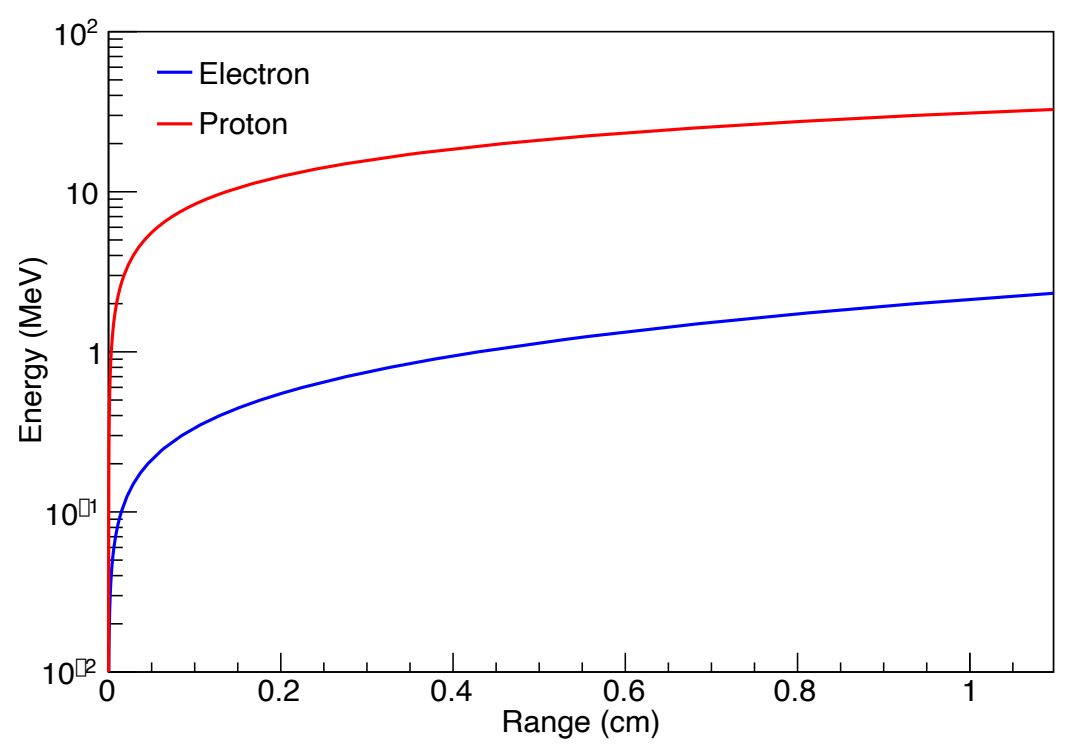

Figure 5.3. Energy vs range for electrons and protons for the ranges of interest for this study. Red denotes protons, blue denotes electrons. The clear separation between electron and proton means it is unlikely a proton hit will be mistakenly identified as an electron hit. Data from [20].

First, the "standard" ArgoNeuT automated reconstruction procedure, including hit finding, hit reconstruction and track reconstruction, as described in detail in [73], was applied. Events were required to have a reconstructed neutrino interaction vertex contained in the fiducial detector volume, defined as $[3,44] \mathrm{cm}$ along the drift direction, $[-16,16] \mathrm{cm}$ vertically from the center of the detector, and $[6,86] \mathrm{cm}$ along the beam.

5.4.1 Signal Selection. In the second step, a low-energy specific procedure to identify and reconstruct isolated hits and clusters was applied. Since low-energy electrons will leave short isolated features in the TPC, hits that are identified as belonging to a reconstructed track were removed. To also remove nearby wire activity associated with a track (such as delta rays), all hits inside a $120^{\circ}$ cone around the first $2.5 \mathrm{~cm}$ of each reconstructed track and a $5 \mathrm{~cm}$ cylinder along the remaining track length were rejected. For tracks reconstructed as being longer than $4 \mathrm{~cm}$, the cylindrical rejection region was extended past the end of the track, in case the automated 
reconstruction cuts the track short. To ensure identical rejection efficiencies for all events, all cut regions in a neutrino event were also cut in the nearby background events dataset. Then, several cuts were made on the remaining hits found in each event. A threshold cut removed hits whose fitted peak height is below a certain ADC count threshold on the induction and collection planes, corresponding to roughly 0.2 $\mathrm{MeV}$. Hits whose fitted peak height is above a maximum ADC count (corresponding to $\sim 1.2 \mathrm{MeV}$ ) were also removed, as they were unlikely to be produced by photon energy depositions. As shown in Figure 5.3, such hits are more likely due to protons. For example, for a proton to travel a distance of $0.4 \mathrm{~mm}$, the wire spacing, it must have a kinetic energy of at least $10 \mathrm{MeV}$, well above the maximum ADC cut. Low energy protons with very short range can result from a neutron-proton reaction on argon, however the FLUKA simulation indicates fewer than $1 \%$ of hits passing cuts are due to protons. A fiducial cut was then applied to remove all hits within $6 \mathrm{~cm}$ of the cathode and anode and hits near corners of the TPC.

To further remove hits likely due to above-threshold electronics noise, matching of hit times between induction and collection planes was required. This plane matching also allowed for reconstruction of 3D hit positions. Using a muon neutrino CC event (shown in Fig. 5.4) as an example, applied cuts are visually demonstrated in Figure 5.5.

After the selection was complete, events were individually handscanned to remove noisy wires and reconstruction failures. Individual wires were removed on an event by event basis if it was clear they had several hits due to electronics noise, with equivalent cuts applied to nearby background events. Some hits were also manually removed if it was clear they belonged to a track that was not reconstructed properly.

Once all cuts were applied and handscanning was complete, the resulting neutrino (background) datasets contained 716 (422) collection plane selected hits. A 


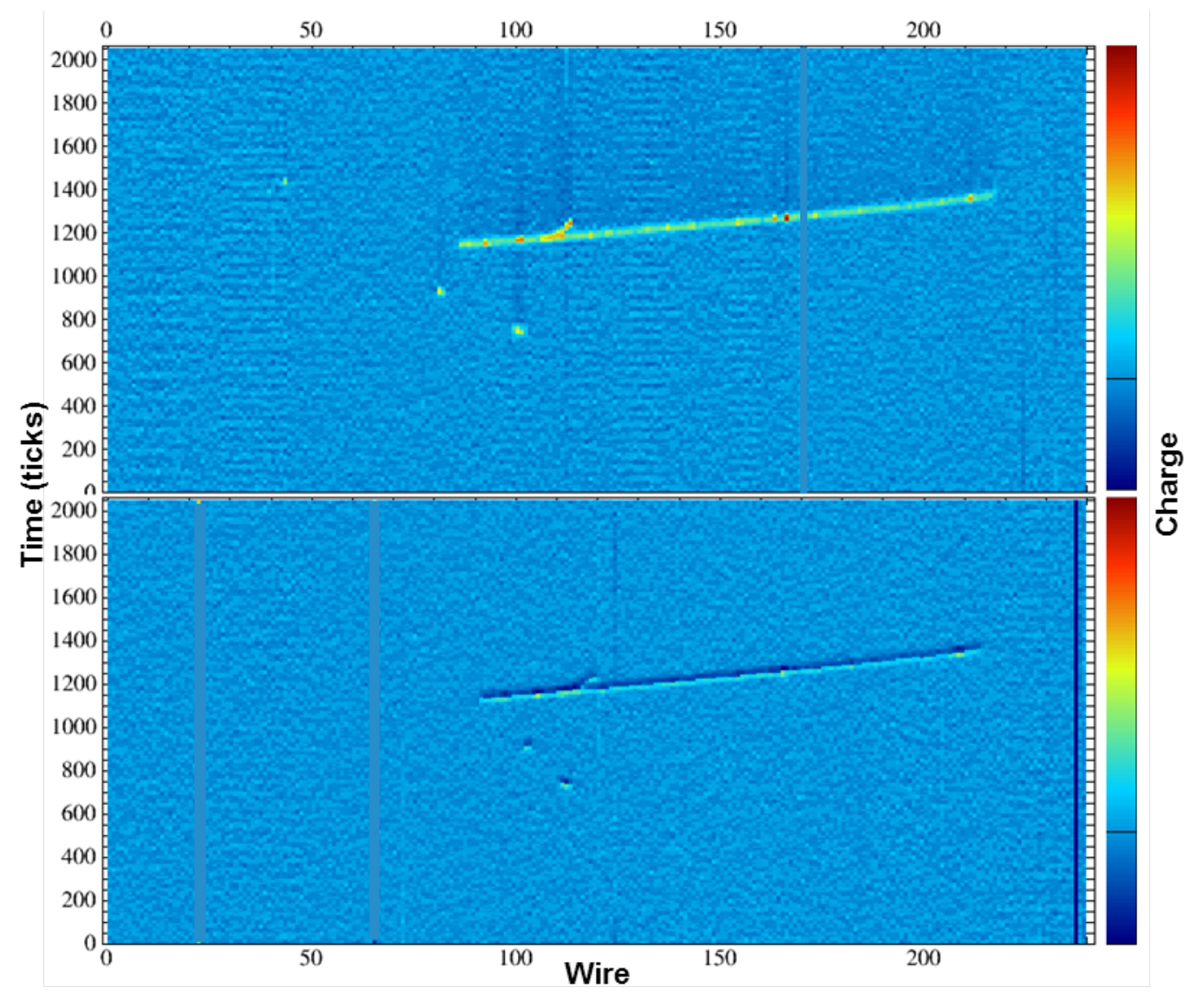

Figure 5.4. A raw neutrino data event with one track reconstructed as a muon and with gamma activity (isolated blips). Top plane is collection, bottom induction. Wire number is indicated on the x-axis. The y-axis indicates time in ticks. Color indicates amount of charge collected.

summary of the level of hit removal achieved in each cut for neutrino, background and MC datasets is found in Table 5.1.

Following this selection, we grouped signal hits into clusters and attempted a reconstruction of clusters' positions and energies. A cluster is defined as a collection of one or more signals on adjacent wires that occur within 40 time ticks on these wires. This value was determined by examining a simulation of electrons with energies in the range of interest. If a cluster spans a noisy or unresponsive wire, each section was considered as a separate cluster. A total number of 553, 4537 and 319 planematched clusters were reconstructed in the selected neutrino, $\mathrm{MC}$ and background 


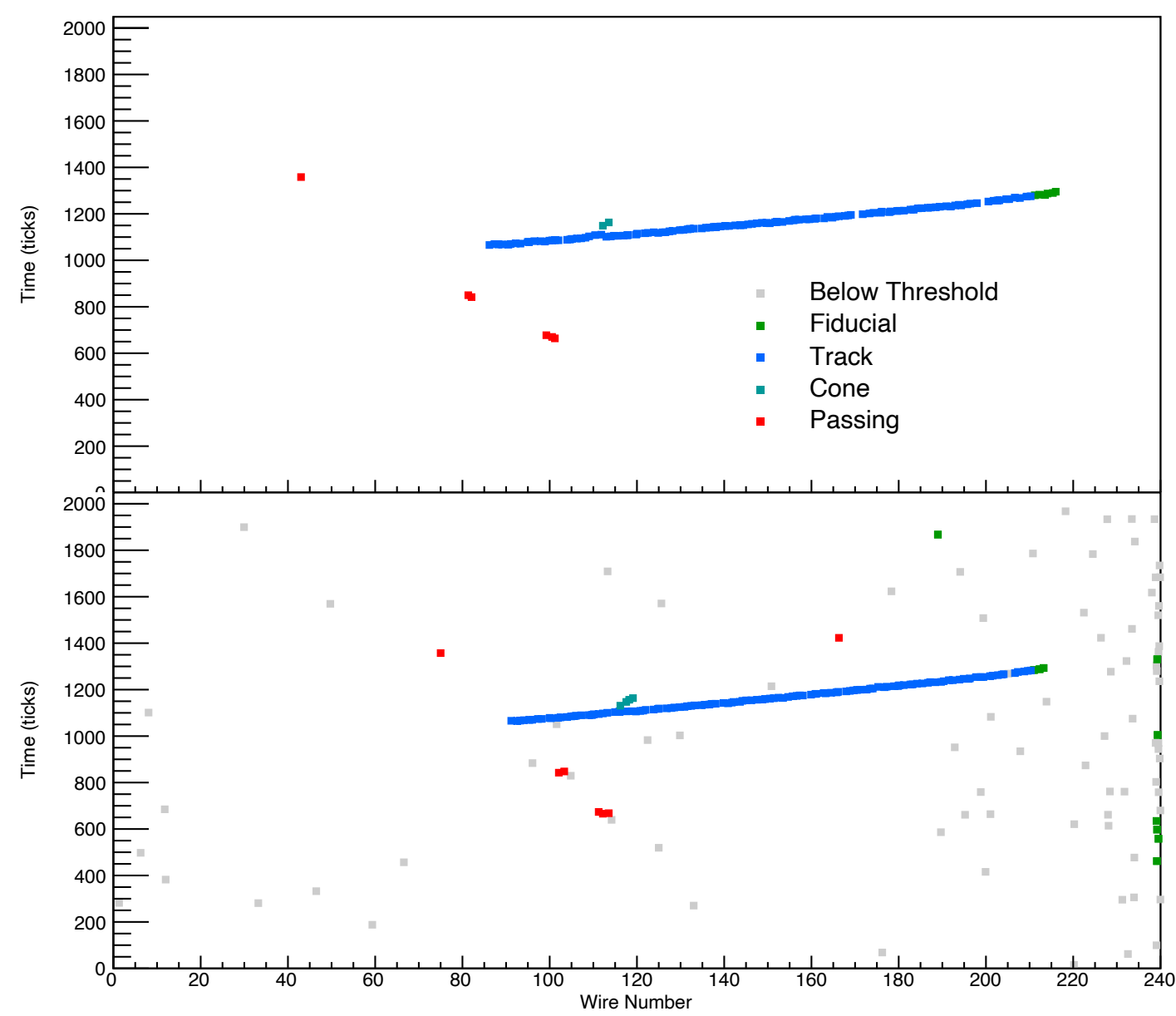

Figure 5.5. The same event as in Figure 5.4 after hit finding and reconstruction. Each square denotes a reconstructed hit. Color indicates whether or not a hit was removed and by which cut (see text). Hits that pass all cuts are in red.

events, respectively. In neutrino events, most of the clusters (75\%) are composed of just one hit, $23 \%$ are two hit clusters, and only $2 \%$ are clusters with more than two hits.

5.4.2 Position Reconstruction. We reconstructed the 3D position of a cluster by matching the furthest upstream collection plane hit in a cluster to the furthest upstream induction plane hit in the matched cluster. This yielded a coordinate on the $y z$-plane. We then included the $x$-coordinate of the collection plane hit to obtain a $3 \mathrm{D}$ position and calculated the distance of each cluster with respect to the neutrino 
Table 5.1. Effectiveness of different cuts for collection plane hits. Cuts are applied sequentially. There are 552 neutrino, 1970 background and $3889 \mathrm{MC}$ events.

\begin{tabular}{cccc}
\hline \hline \multirow{2}{*}{ Cut } & \multicolumn{3}{c}{ Percent of Hits Remaining } \\
\cline { 2 - 4 } & Neutrino & Background & MC \\
\hline Minimum Peak Height & $65 \%$ & $38 \%$ & $94 \%$ \\
Maximum Peak Height & $58 \%$ & $37 \%$ & $84 \%$ \\
Plane Matching \& Handscanning & $24 \%$ & $10 \%$ & $54 \%$ \\
\hline
\end{tabular}

interaction vertex. While a cluster may span more than one wire in a plane, the distance traveled by the particle creating the cluster is negligible when compared to the distance from the vertex.

5.4.3 Charge to Energy Conversion. To reconstruct the energy associated with each reconstructed cluster, first the measured pulse area $(\mathrm{ADC} \times$ time $)$ of each hit was converted to the number of electrons by an electronic calibration factor, then a lifetime correction was applied to account for ionization electron loss due to attachment on impurities in the liquid argon, as described in [73].

Calorimetric reconstruction in a LArTPC requires converting collected charge to the original energy deposited. This requires applying a recombination correction which depends on charge deposition per unit length $d Q / d x$ [15], as described in Sec. 3.5. The low-energy photon-induced signals in the present analysis are just isolated clusters of a small number of hits, not extended tracks, so the effective length of the track seen by a wire cannot be determined.

A different method to estimate the energy from the deposited charge which relies on the assumption that all hits passing cuts are due to electrons only has been developed; simulation indicates fewer than $1 \%$ of hits passing cuts are due to other 
particles. The method uses the NIST table of track lengths for electrons at various energies (ESTAR) [20], from $10 \mathrm{keV}$ to $1 \mathrm{GeV}$. Using this table, we can calculate $d E / d x$ values by dividing the energy by the track length for each row in the table. Using the Modified Box Equation (Eq. 3.7) to model the recombination effect, we can calculate the expected $d Q_{\text {coll }} / d x$ and by multiplying by the track length (i.e. $d x$ ), we obtain the expected amount of charge collected for an electron at a given energy, as shown in Figure 5.6. By using the result of a fit, also shown in the Figure, we can convert the measured collected charge on each individual hit to deposited energy. The total energy in a cluster is the sum of the deposited energy reconstructed for each individual hit forming the cluster. To test the accuracy of this method, we applied it to a sample of GEANT4 simulated electrons in the energy range of interest. Figure 5.7 indicates that it works remarkably well, with an energy reconstruction bias of less than 20\%, negligible compared to the uncertainty in the functional form of the recombination correction itself. Applying this method to a FLUKA simulation of one-hit electron clusters results in the energy spectrum shown in Figure 5.8, where almost all (99.9\%) one-hit clusters have a reconstructed energy below $2.5 \mathrm{MeV}$.

5.4.4 Systematic Uncertainties. There are three primary sources of systematic uncertainty associated with hit and energy reconstruction in this analysis. As the electron lifetime varies between runs, we expect a variation and uncertainty in the number of near-threshold hits that are selected as signal. Despite having precise measurements of electron lifetime for all runs, we conservatively account for electron lifetime uncertainties by re-running FLUKA signal hit selections with a $25 \%$ increase in either high- or low-lifetime runs; the resultant spread in reconstructed multiplicities and energies is treated as the systematic uncertainty from this source. A second systematic uncertainty arises from the choice of a true underlying functional form for the recombination correction. To account for this uncertainty, we consider reconstruction of simulated events using the unmodified Box Model as described in [16]; 


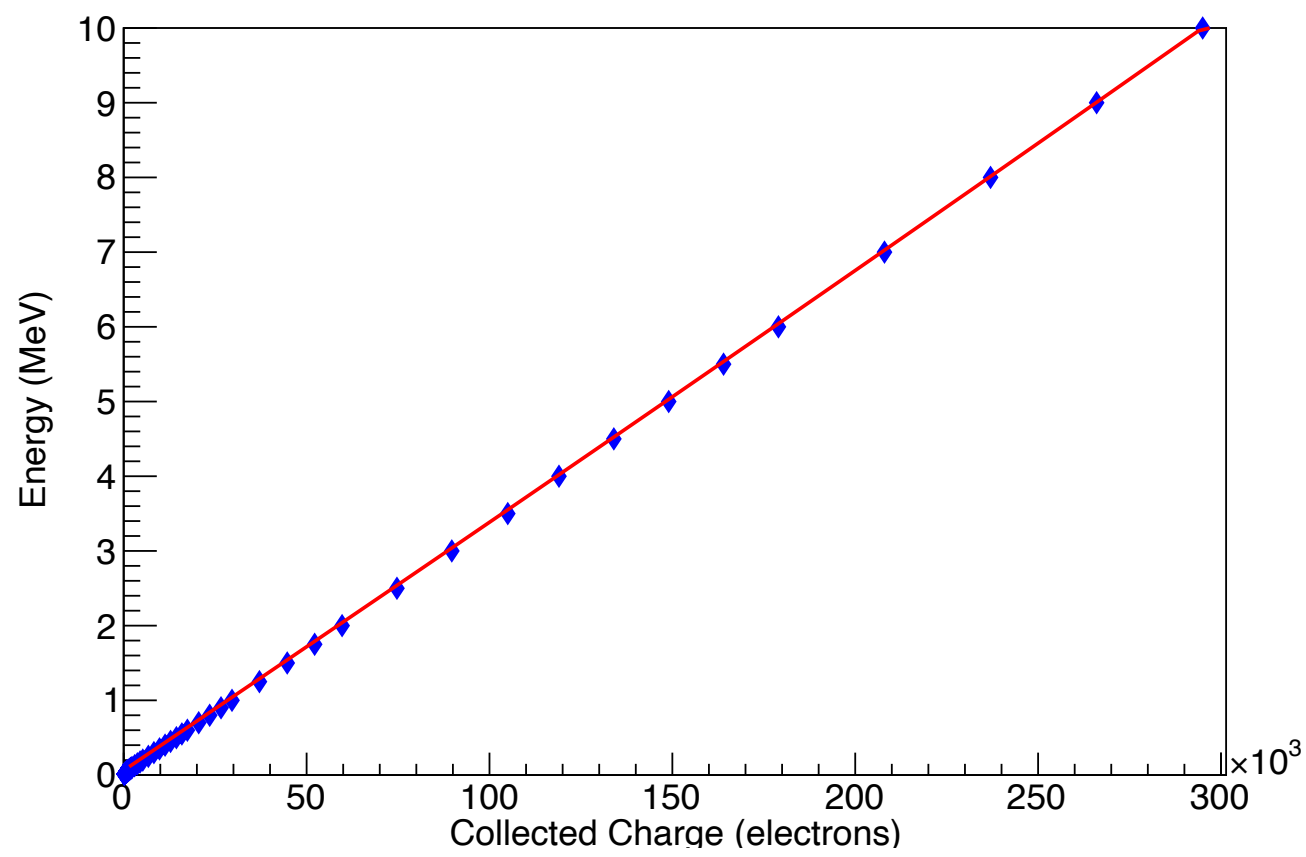

Figure 5.6. Energy deposited vs collected charge. Line indicates fit used to perform energy calculations from collected charge.

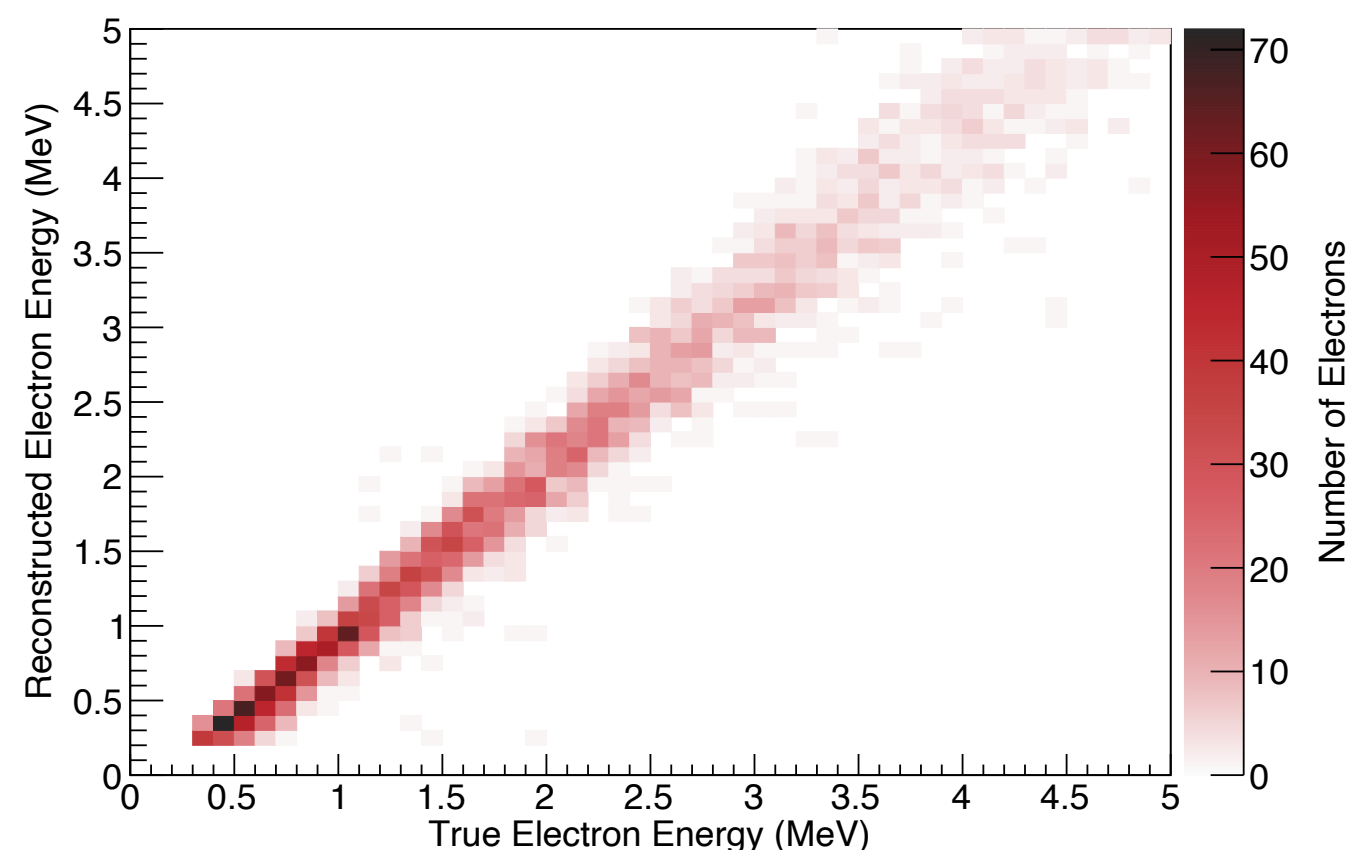

Figure 5.7. Reconstructed energy vs true electron energy using the charge method for a sample of simulated electrons with energies between 0 and $5 \mathrm{MeV}$. Events where the electron was not detectable are excluded. 


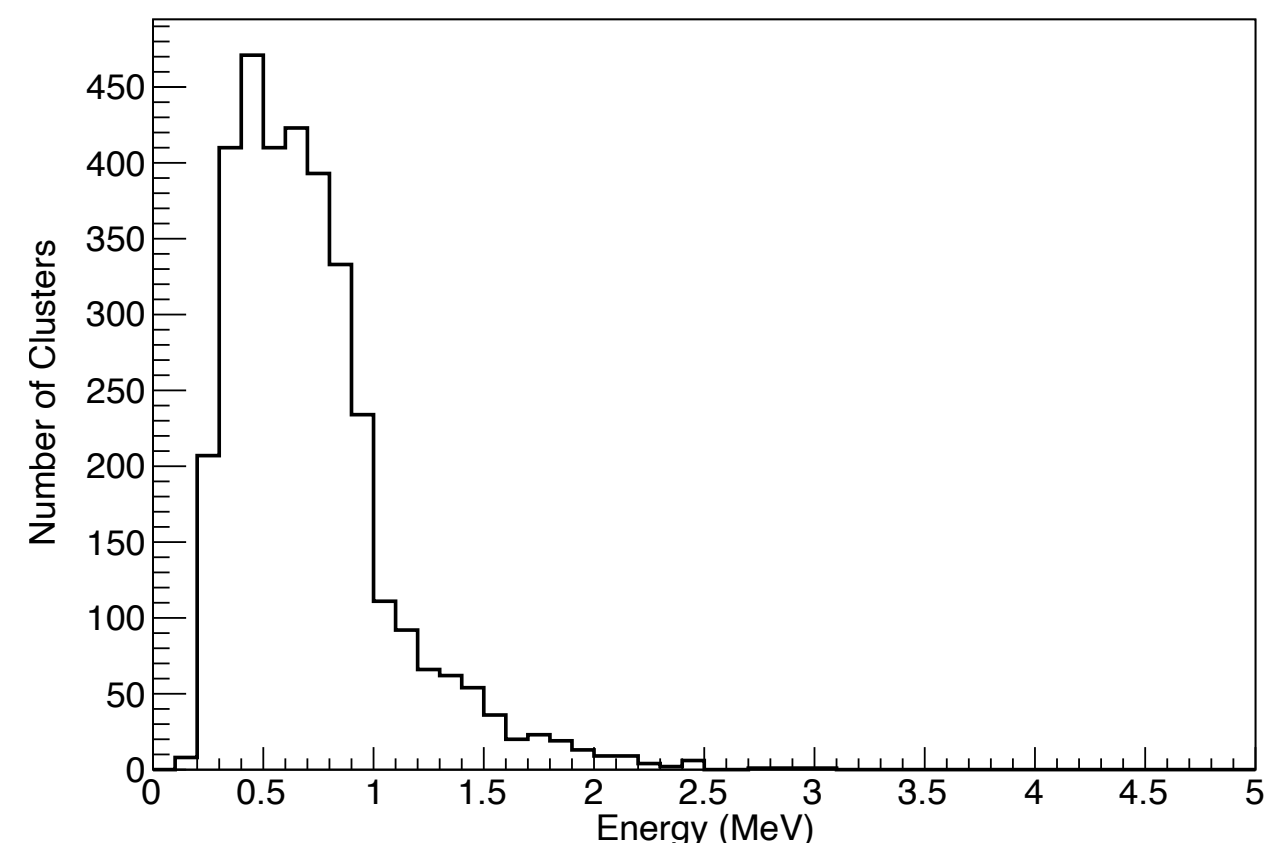

Figure 5.8. Energy deposited in individual one-hit clusters according to the FLUKA simulation.

deviation from the default selection is treated as an uncertainty contribution from this source. Finally, there is a $3 \%$ error associated with the utilized muon-based calibration constants, which are fully correlated between all runs. Any multiplicity or energy variation arising from a $\pm 3 \%$ shift in thresholds and reconstructed energies is treated as an uncertainty from this source. We find that electron lifetime uncertainties dominate the low bins of multiplicity and energy, while calibration constants are the dominant systematic uncertainty at higher photon energy deposition. Systematic uncertainties in reconstructed positions are expected to be small and were not considered in this analysis.

\subsection{Results}

5.5.1 Comparison of Neutrino and Background Datasets. Table 5.2 shows a comparison of neutrino and background datasets. Comparing the different metrics leads to the conclusion that we have observed a statistically significant sample of 
neutrino-induced $\mathrm{MeV}$-scale photons. Hit and cluster multiplicities are found to be significantly higher in the neutrino dataset than in the background dataset, with $1.30 \pm 0.07$ and $0.21 \pm 0.02$ hits per event, respectively. This difference corresponds to a $15 \sigma$ statistical excess of signal in the neutrino dataset. The higher neutrino dataset multiplicity is also accompanied by a larger per-event signal occupancy ( $54 \pm 4 \%$ in neutrino events versus $12 \pm 2 \%$ in background events) and total signal energy per event (1.1 MeV in neutrino events versus $0.19 \mathrm{MeV}$ in background events). This can be interpreted as evidence of neutrino-induced MeV-scale energy depositions.

Table 5.2. Comparison of neutrino and background datasets when examining hits passing all cuts. The difference in the first four metrics indicates neutrino-induced $\mathrm{MeV}$-scale activity is visible.

\begin{tabular}{ccc}
\hline \hline Metric & Neutrino Data & Background \\
\hline Number of hits per event & 1.30 & 0.21 \\
Number of clusters per event & 1.00 & 0.16 \\
Average total signal energy in an event (MeV) & 1.11 & 0.19 \\
Percent of events with at least one signal hit & $54 \%$ & $12 \%$ \\
Average cluster distance from vertex $(\mathrm{cm})$ & 22.4 & - \\
\hline
\end{tabular}

5.5.2 Comparison to MC Simulations. A comparison of reconstructed perevent signal multiplicity and total signal energy for data and FLUKA MC simulation are shown in Figure 5.9, top and bottom respectively. Background activity is added to $\mathrm{MC}$ events on an event-by-event basis. The activity from a randomly selected background event is added to each MC event.

In both data and $\mathrm{MC}$, around half of the events have no signal clusters, as expected based on the small ArgoNeuT detector size and the previously-mentioned sizable number of predicted product nuclei in the ground-state. Overall, there is good 
agreement between data and FLUKA MC predictions. We find a $\chi^{2} /$ ndf of $7.81 / 12$ ( $\mathrm{p}$-value 0.80 ) for the total reconstructed energy distributions, and a $\chi^{2} / \mathrm{ndf}=12.6 / 6$ (p-value 0.05) for the cluster multiplicity distribution. Thus, we observe that FLUKA, which incorporates low-level nuclear processes that result in the production of $\mathrm{MeV}$ scale energy depositions following interactions of $\mathrm{GeV}$-scale neutrinos in liquid argon, agrees well with the data. We observe that the largest contributor to the $\chi^{2}$ between the data and MC multiplicity distributions is the difference in high-multiplicity events. The modest excess in MC, which spreads over multiple reconstructed energy bins, could be indicative of flaws in the hit selection process, or of imperfections in models or libraries utilized by FLUKA. This feature can be better examined in future highstatistics studies in larger LArTPCs. Finally, we notice a dip in the first bin in Figure 5.9 (bottom), due to detector thresholding, which can vary in data from event to event due to different electron lifetime values.

Both components - de-excitation photons and photons produced by interactions of final-state neutrons on argon - are needed to have data-MC agreement. Figure 5.11 demonstrates the impact of removing either of these components on the cluster multiplicity and total energy distributions shown previously. The top row indicates the effect of removing neutron-produced photons, and the bottom row indicates the effect of removing deexcitation photons. In all four plots, agreement between data and $\mathrm{MC}$ is shown to be poor. If deexcitation photons are removed from FLUKA distributions, we obtain a $\chi^{2} / \mathrm{ndf}=82.6 / 12$ for reconstructed energy and $\chi^{2} / \mathrm{ndf}=93.8 / 6$ for the cluster multiplicity. If neutron-produced photons are removed, we obtain $\chi^{2} /$ ndf $=194 / 12$ and $\chi^{2} /$ ndf $=197 / 6$ for these same distributions, respectively. To confirm this, we also compared ArgoNeuT data with a different neutrino interaction generator, GENIE [86], commonly used by US-based neutrino experiments; existing user interfaces allowed for easy generation of GENIE final states within the LArSoft framework. The same event selection and reconstruction procedure as in FLUKA was 

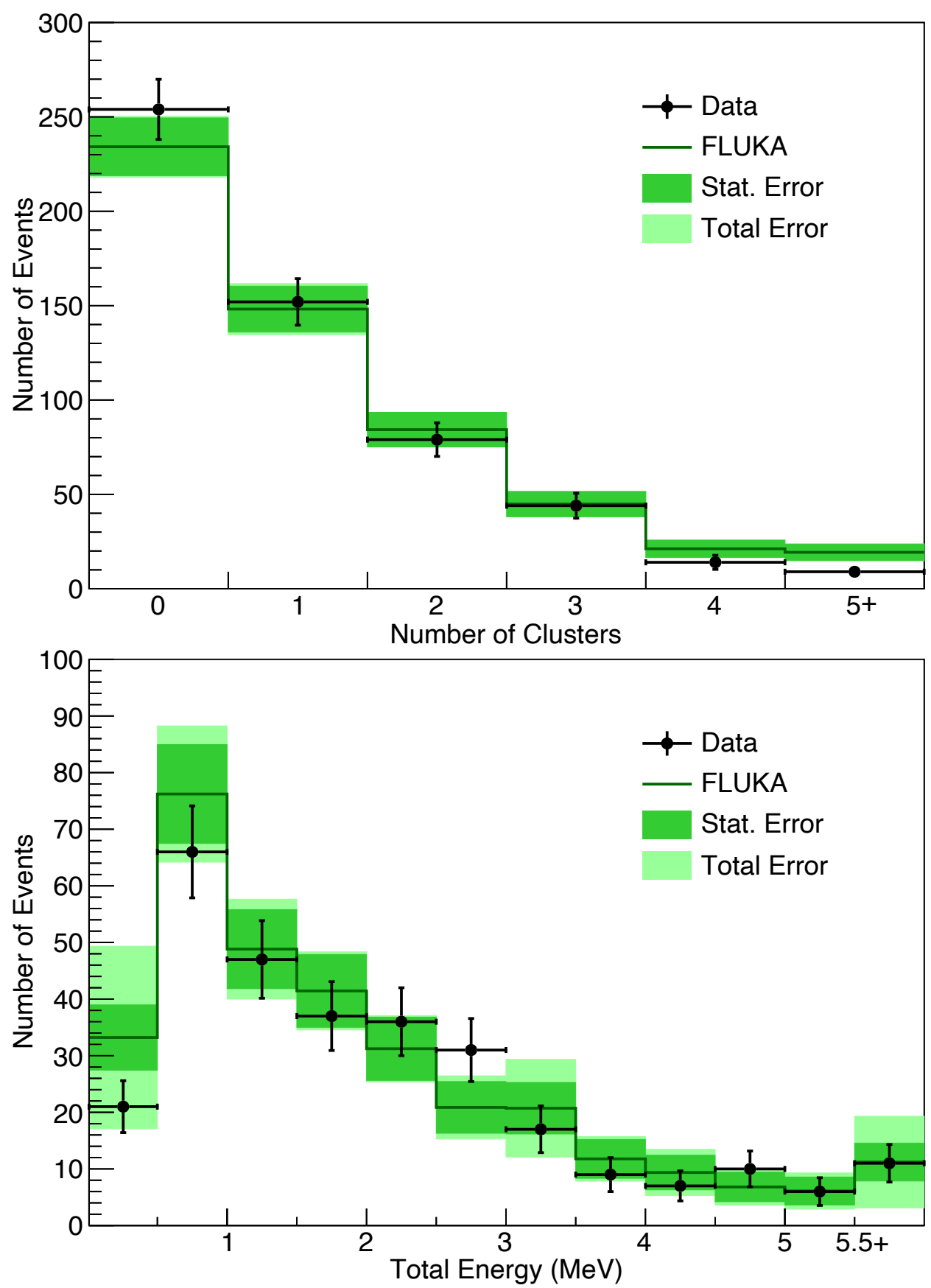

Figure 5.9. Cluster multiplicity (top) and total signal reconstructed energy (bottom) in an event for neutrino data and FLUKA MC events. Events with no reconstructed energy are not included. Data points include statistical error. Dark green line indicates FLUKA prediction with data-driven background added (see text). Dark green shaded area is statistical error in FLUKA, overlaid on total error (statistical + systematic) for FLUKA in light green shading. MC is normalized to the number of neutrino data events. 
applied to GENIE events. As an example, a comparison of reconstructed multiplicity is shown in Figure 5.10 (top). The $\chi^{2} /$ ndf is $57.9 / 6$. This disagreement is attributed to the lack of de-excitation photons in the GENIE simulation of neutrino-argon interactions.

These results indicate that the observed MeV-scale signals in ArgoNeuT contain both de-excitation and neutron-produced photons. The contribution of each of these sources to the total activity in an event as given by the FLUKA simulation is shown in Table 5.3. We find that we cannot distinguish between the two sources of photons by examining the energy of a hit or cluster alone, but we do see a difference in the distance of a cluster with respect to the neutrino interaction vertex. The distribution of these distances is seen in Figure 5.12. Photons produced by de-excitation of the final-state nucleus tend to be concentrated at lower distances, while photons produced by inelastic neutron scattering dominate at higher distances.

Table 5.3. Relative contributions of de-excitation and neutron-produced photon components in FLUKA MC.

\begin{tabular}{cccc}
\hline \hline Metric & De-excitation & Neutron & Total \\
\hline Number of hits per event & 0.48 & 0.98 & 1.46 \\
Number of clusters per event & 0.35 & 0.77 & 1.12 \\
Average event energy (MeV) & 0.41 & 0.76 & 1.17 \\
Average cluster energy (MeV) & 1.18 & 0.98 & 1.04 \\
Average hit energy (MeV) & & 0.86 & 0.80 \\
Average cluster distance from vertex (cm) & 15.7 & 23.4 & 21.0 \\
\hline
\end{tabular}



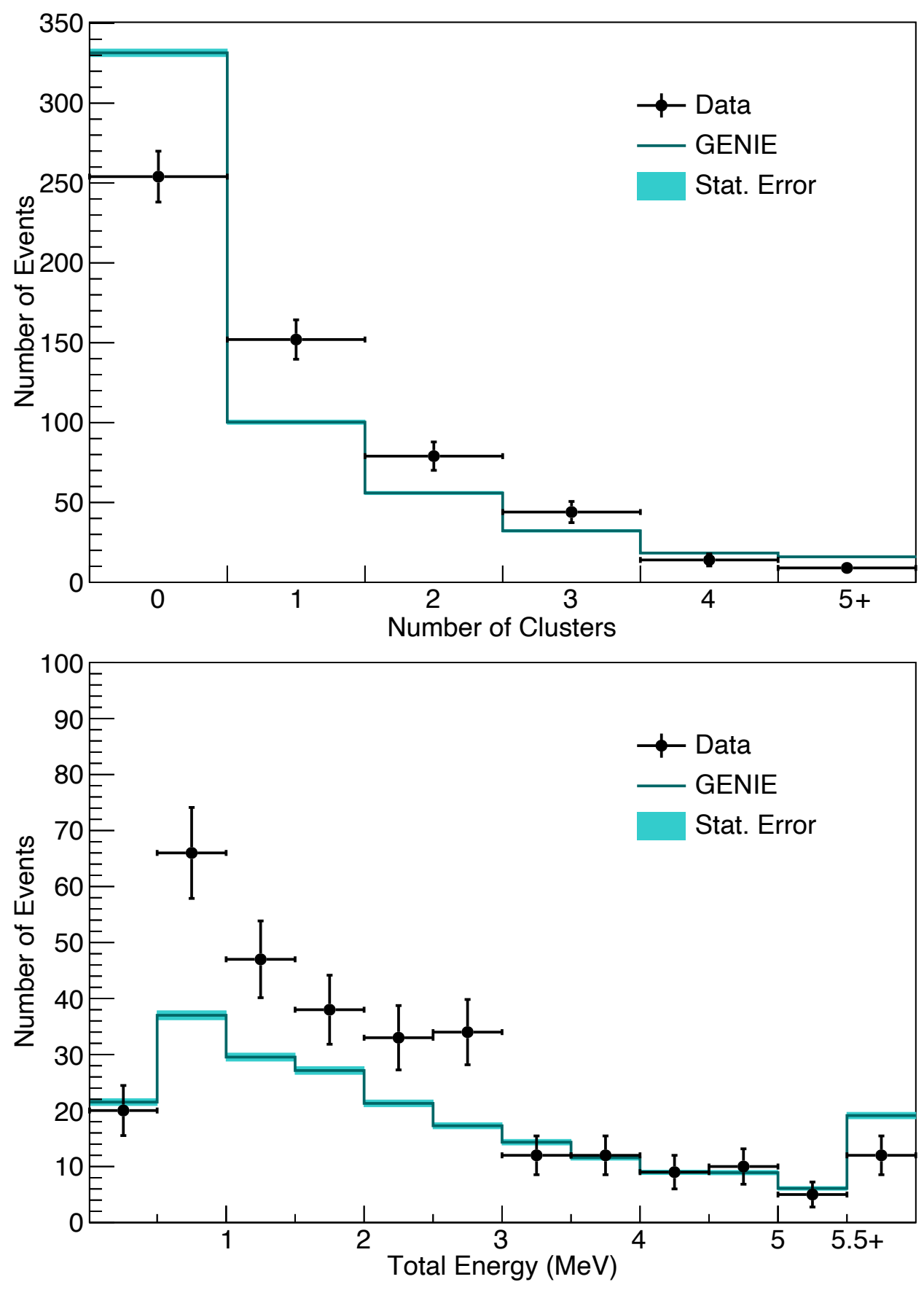

Figure 5.10. Cluster multiplicity (top) and total signal reconstructed energy (bottom) in an event for neutrino data and GENIE MC events. Events with no reconstructed energy are not included. Data points include statistical error. Dark blue line indicates GENIE prediction. Light blue shaded area indicates statistical error for GENIE prediction. MC is normalized to the number of neutrino data events. 

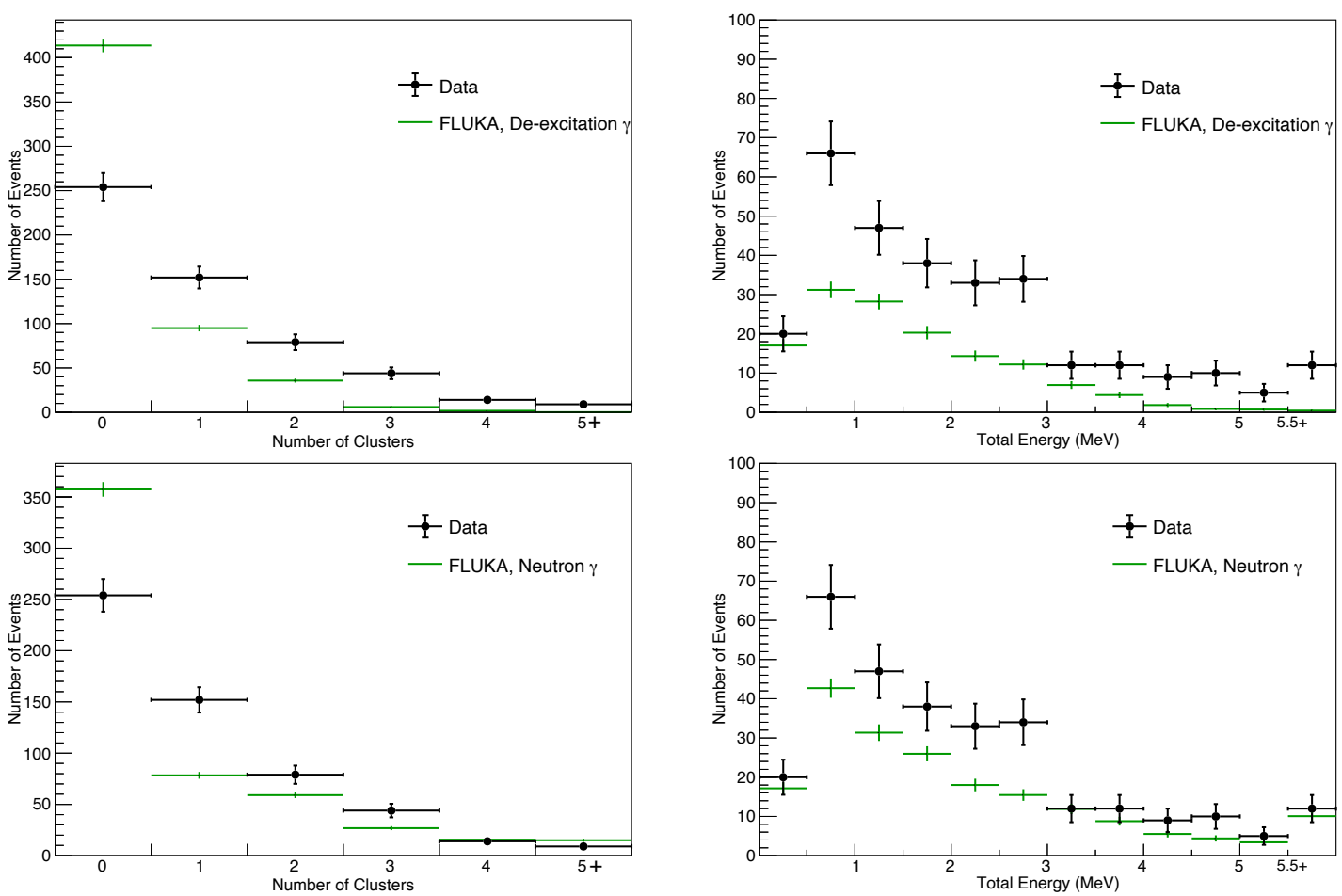

Figure 5.11. Cluster multiplicity (left) and total signal reconstructed energy (right) in an event for neutrino data and FLUKA MC events. Events with no reconstructed energy are not included. Data points include statistical error. Dark green indicates FLUKA prediction with only deexcitation photons (top) or with only neutronproduced photons (bottom). Data-driven background is added to all plots (see text). MC is normalized to the number of neutrino data events. 


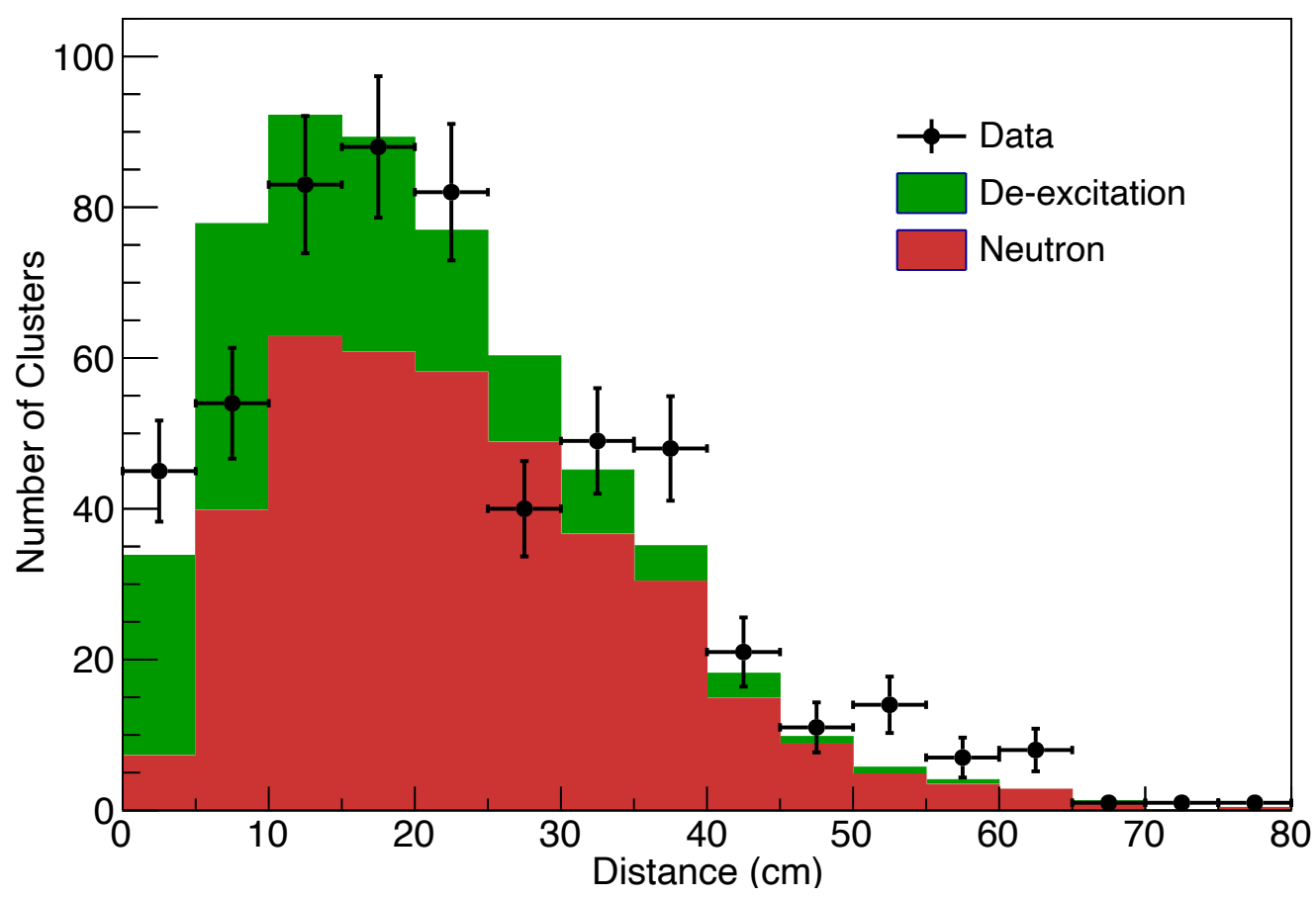

Figure 5.12. Distributions of cluster position with respect to the neutrino interaction vertex in neutrino data and FLUKA MC events. Data includes statistical error. Green indicates the contribution of photons from de-excitation of the final-state nucleus. Red indicates the contribution of photons from inelastic neutron scattering. $\mathrm{MC}$ is area normalized to data.

\subsection{Conclusions}

The ability to reconstruct activity at the MeV scale in a LArTPC is crucial for future studies of supernova, solar, and beam neutrino interactions. Future MC studies and higher-statistics datasets from future large LArTPCs will provide additional understanding of the value of these $\mathrm{MeV}$-scale features. By studying low-energy depositions produced by photons in ArgoNeuT neutrino interactions and comparing to simulation, we have shown that such a reconstruction is possible. Performing this study required the creation of new techniques for low-energy LArTPC reconstruction. By reconstructing photons produced by nuclear de-excitation and inelastic neutron scattering, we have extended the LArTPC's range of physics sensitivity down to the sub-MeV level, reaching a threshold of $0.3 \mathrm{MeV}$ in this analysis. This range now 
spans more than three orders of magnitude, up to the GeV level.

This analysis represents the first-ever reported detection of de-excitation photons or final-state neutrons produced by beam neutrino interactions in argon. Both of these particle classes could provide valuable new avenues of investigation for physics reconstruction in LArTPCs. Reconstruction of MeV-scale neutron-produced features may enable some level of direct reconstruction of final-state neutron energies or multiplicities, which would provide a valuable new handle on one of the dominant expected differences between neutrino and antineutrino interactions in liquid argon. Precise reconstruction of de-excitation photon multiplicities and energies will improve overall reconstruction of neutrino energies, particularly for those at lower energies, such as supernova and solar neutrinos.

In addition, studies of low scale new physics scenarios, such as millicharged particles, light mediators, and inelastic scatterings with small splittings (see e.g. Refs. $[62,87,88])$, could invaluably profit from such low energy reconstruction, as shown in the next chapter. 


\section{CHAPTER 6}

\section{MILLICHARGED PARTICLE SEARCH USING ARGONEUT}

As discussed in Section 2.8, millicharged particles are one of many BSM physics theories being investigated in experimental particle physics today. Using the techniques for reconstructing low energy activity in LArTPCs, described in Chapter 5, it is possible to perform a search for millicharged particles using a LArTPC. This chapter presents the techniques used in and the results of such a search using ArgoNeuT data. This content, which represents the first BSM search ever performed with a neutrino LArTPC, has been submitted to Physical Review Letters and is found in arXiv:1911.07996 [89]. As in Chapter 5, this work is based on my own analysis efforts, building on low-level tools developed by the ArgoNeuT collaboration and working with Fermilab theorists.

\subsection{Production of Millicharged Particles Reaching ArgoNeuT}

Millicharged particles can be produced at any intense fixed-target-produced beam via the decays of neutral mesons or direct Drell-Yan pair production arising from proton interactions in the target. (See Sec. 2.8.) [21,62]. A detector placed downstream of such a target may be exposed to a large flux of millicharged particles produced at the target. In the case of ArgoNeuT, the detector is placed downstream of the NuMI target. The NuMI target is struck by $120 \mathrm{GeV}$ protons with a high intensity. The expected flux of millicharged particles entering ArgoNeuT, $1 \mathrm{~km}$ away from the target, is given in Figure 6.1 [21]. The Figure gives the flux of millicharged particles from various meson decays and Drell-Yan production for charges of $10^{-2} e$ with an exposure of $10^{20}$ POT. Millicharged particles are produced in pairs, so the maximum mass for a millicharged particle is half the parent's mass. This plot demonstrates 
that even a small detector like ArgoNeuT, placed $1 \mathrm{~km}$ away from the source can still receive a large number of millicharged particles.

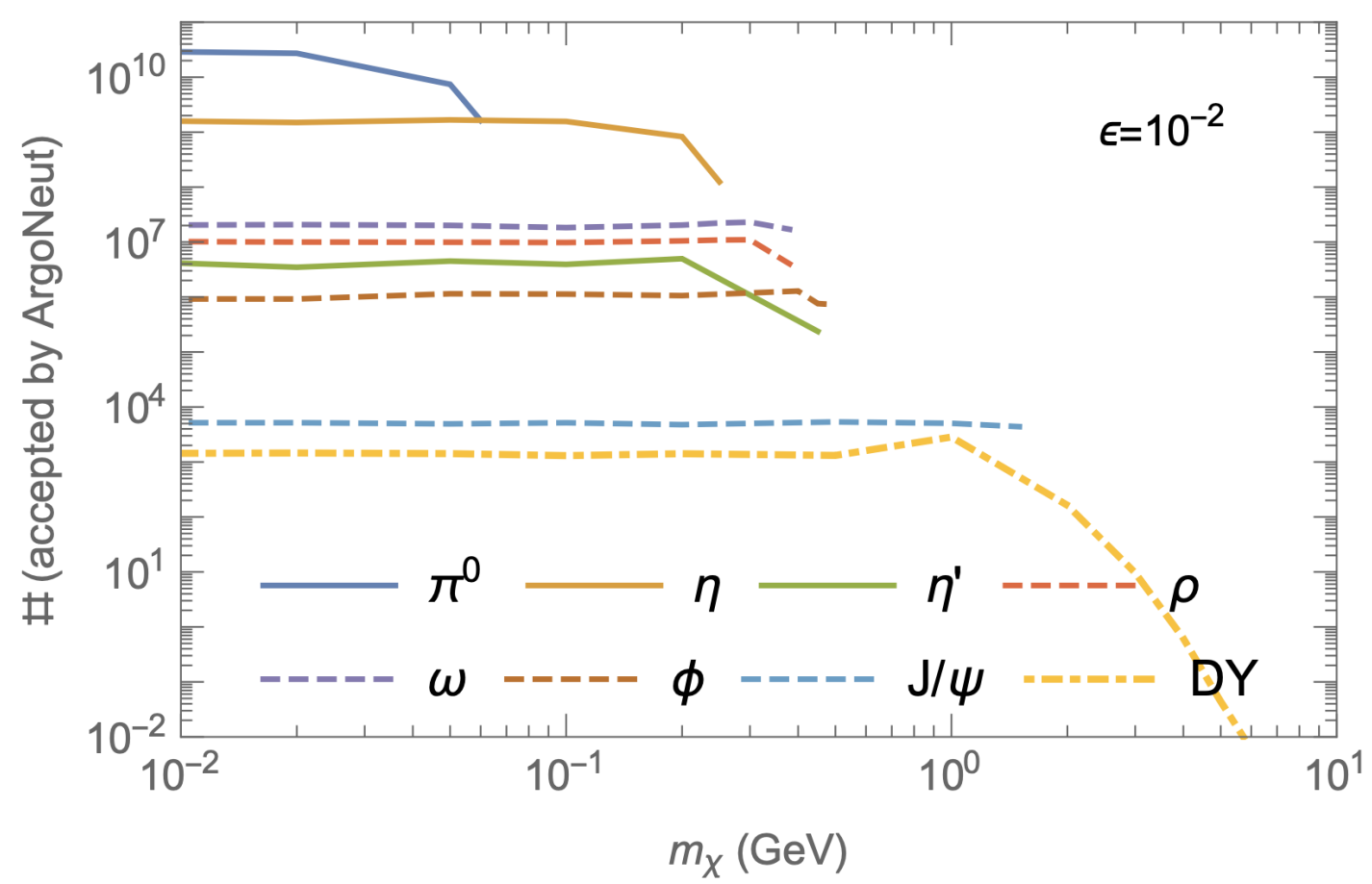

Figure 6.1. Number of millicharged particles with charge $10^{-2} e$ entering ArgoNeuT for $10^{20}$ POT, integrated over all millicharged particle energies. Colors indicate which neutral meson decay. Yellow dot-dash indicates Drell-Yan production. From [21].

\subsection{Detection Signature}

The detection signature of mCPs in the detector is elastic scattering with atomic electrons resulting in knock-on recoils above the detection threshold [21]. Therefore, in order to be able to reconstruct mCPs which pass through ArgoNeuT, we search for small individual energy depositions in the detector. As demonstrated in Chapter 5, in ArgoNeuT it is possible to reconstruct with very good efficiency electromagnetic energy depositions as low as $300 \mathrm{keV}$. Following the method suggested in [21], to cut down on possible backgrounds in our search for mCPs we look for events with two individual soft energy depositions that are aligned with the upstream target, as shown in Figure 6.2. 
Signal

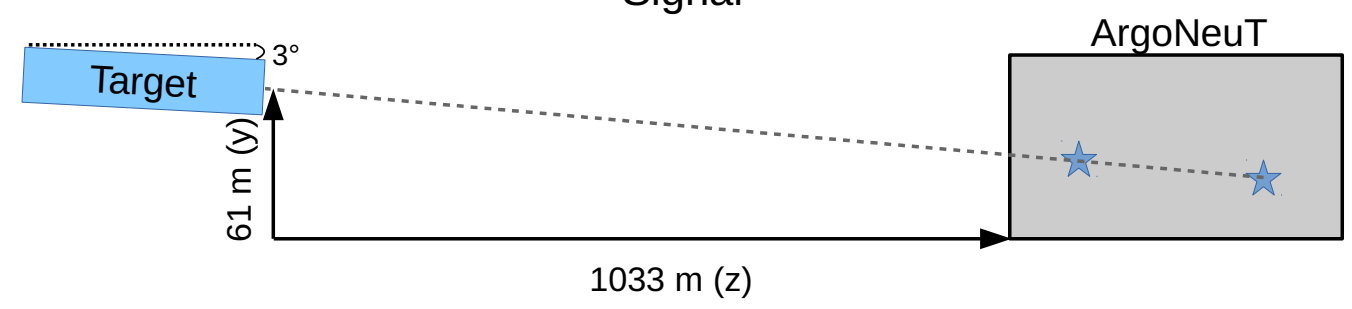

\section{Background}

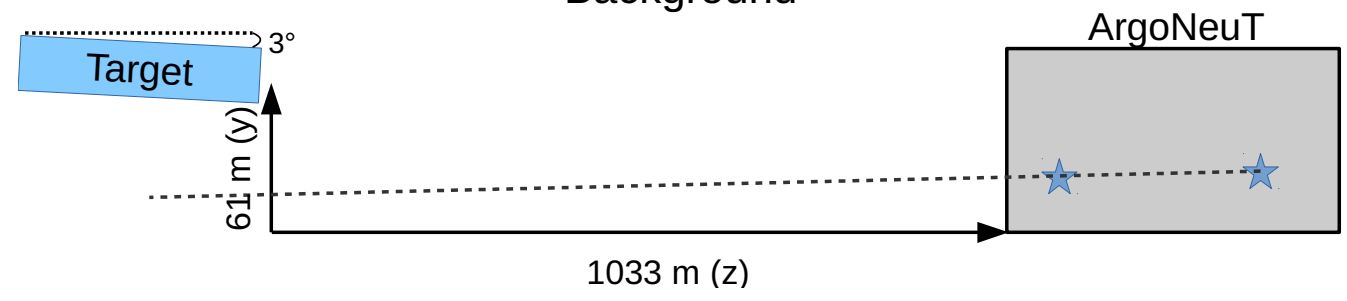

Figure 6.2. Schematic (not to scale) of the ArgoNeuT detector location relative to the upstream target. Signal is a double-hit event with a line defined by the two hits pointing to the target (top). A background double-hit event generally will not point to the target (bottom). Figure adapted from Ref. [21].

\subsection{Datasets}

We searched for the presence of mCPs in data from ArgoNeuT's antineutrino mode run. The trigger condition for the ArgoNeuT data acquisition was set in coincidence with the NuMI beam spill signal. A total of 4,056,940 collected triggers has been analyzed. The vast majority of NuMI beam spills delivered did not produce an observable neutrino interaction within the TPC due to the very low neutrino cross-section and the limited size of the detector, resulting in "empty" events. In this analysis we searched for the possible presence of mCPs in these empty events. Events containing a neutrino interaction inside the LAr volume and events contain- 
ing charged particles (mainly muons) produced by neutrino interactions upstream of the ArgoNeuT detector and propagating through the LAr TPC volume are removed. The background for the $\mathrm{mCP}$ search is due to ambient gamma ray activity, beta electrons from intrinsic ${ }^{39} \mathrm{Ar}$ activity, fluctuations of electronics noise faking signals from true energy depositions, and low-energy electrons produced by Compton scattering of photons from inelastic scattering of entering neutrons from neutrino interactions occurring upstream of the detector. To estimate the contribution due the first three sources of background, in the following analysis we compare events acquired when the NuMI beam was operating at its typical high intensity (named "high-beam" in the following) to events acquired when the intensity was very low $(<1 \%$ of the average intensity, named "low-beam" in the following). In this case the last source of background, coming from neutrino-induced neutrons, is not present.

\subsection{Reconstruction}

The reconstruction technique used in this analysis is described in detail in Chapter 5. It consists of a two step process, the standard LArTPC reconstruction [73] followed by a specific procedure for the identification of isolated low-energy depositions in the event. In the first stage of the analysis, hits in the recorded TPC wire signals are found, and clusters of consecutive hits are identified. Events with highenergy activity, i.e. with long tracks or showers, are removed. This leaves 3,259,427 high-beam events, corresponding to $1.0 \times 10^{20}$ protons on target $(\mathrm{POT})$, and 208,730 low-beam events. The next step aims at efficiently identifying and reconstructing isolated low-energy activity in the selected events. Only hits localized in space within a fiducial volume region are selected, and a series of cuts is applied to possibly remove random electronics noise, as described in detail in Chapter 5.. Individual signal hits whose amplitude corresponds to an energy deposition of $>300 \mathrm{keV}$ are grouped into clusters, where a cluster is defined as one or more hits on adjacent wires. For each 
cluster on a wire plane, we look for a corresponding cluster on the other wire plane that appears at the same time, a process called plane matching. Plane matching keeps hits due to true energy depositions in the TPC volume and rejects hits due to electronic noise fluctuations above threshold occurring in either plane but not simultaneously in both. This technique is used to significantly reduce random electronics noise. Plane matching also allows for a determination of the three-dimensional (3D) position of the cluster.

\subsection{High-Beam vs Low-Beam Data}

The selected clusters appear to be uniformly distributed throughout the detector volume. The average number of low-energy clusters per event is 0.15 and 0.069 for high-beam events and low-beam events respectively. Cluster multiplicities are given in Table 6.1. The vast majority of the events are empty (0 clusters) in both data sets, with a lower fraction $(88 \%)$ in the high-beam data. In the low-beam data (94\% empty events), the 1-cluster fraction ( $\sim 6 \%$, mainly from ${ }^{39} \mathrm{Ar} \beta$ activity) almost exhausts the sample. The greater activity in the high-beam sample is expected to be from neutrino-produced neutrons entering the detector volume. Additional activity from low energy electrons can be anticipated to be produced by elastic interactions of mCPs generated at the neutrino beam production target. Since our analysis method of selecting multiple soft energy depositions aligned with the upstream target is expected to be very effective in reducing the background [21], we do not apply any background subtraction procedure to the data.

\subsection{Analysis}

The final step of the analysis, the search for possible mCPs in events from the high-beam data, requires the identification of two low-energy depositions that are aligned with the upstream target (see Fig. 6.2 top). The distribution of the energy 
Table 6.1. Number of plane matched clusters passing cuts in an event for events with low-beam intensity ("low-beam") and events without high-energy activity ("highbeam").

\begin{tabular}{ccccc}
\hline \hline Number & Events & Percentage & Events & Percentage \\
of clusters & low-beam & low-beam & high-beam & high-beam \\
\hline 0 & 195,420 & $93.6 \%$ & $2,855,299$ & $87.6 \%$ \\
1 & 12,428 & $5.95 \%$ & 330,548 & $10.1 \%$ \\
2 & 782 & $0.37 \%$ & 55,372 & $1.70 \%$ \\
3 & 76 & $0.036 \%$ & 13,177 & $0.40 \%$ \\
$\geq 4$ & 24 & $0.011 \%$ & 5,031 & $0.15 \%$ \\
\hline
\end{tabular}

deposited in each cluster, in each one-hit cluster and the distance between clusters for events with at least two clusters is shown in Figure 6.3. As shown in the top left figure, the majority of events have energy depositions in the region around $1 \mathrm{MeV}$. For events with at least two clusters we create all possible lines that connect the two clusters. While many events have more than two clusters, we find that no lines have more than two collinear points within a tolerance of $3 \mathrm{~cm}$ (i.e. there are no threecluster lines). To check whether the lines point back to the target we extrapolate every line to a plane located at the downstream end of the target (1033 m upstream) and normal to the neutrino beam direction.

The uncertainty on the location of the intersection of the line with the plane is determined by the separation of the clusters (smaller cluster spacing corresponds to larger uncertainties) and stems from the uncertainties in the locations of the clusters inside the detector. The latter uncertainties are determined by the spatial resolution of the detector, which is $0.015 \mathrm{~cm}$ in the horizontal drift direction $(x), 0.28 \mathrm{~cm}$ in 

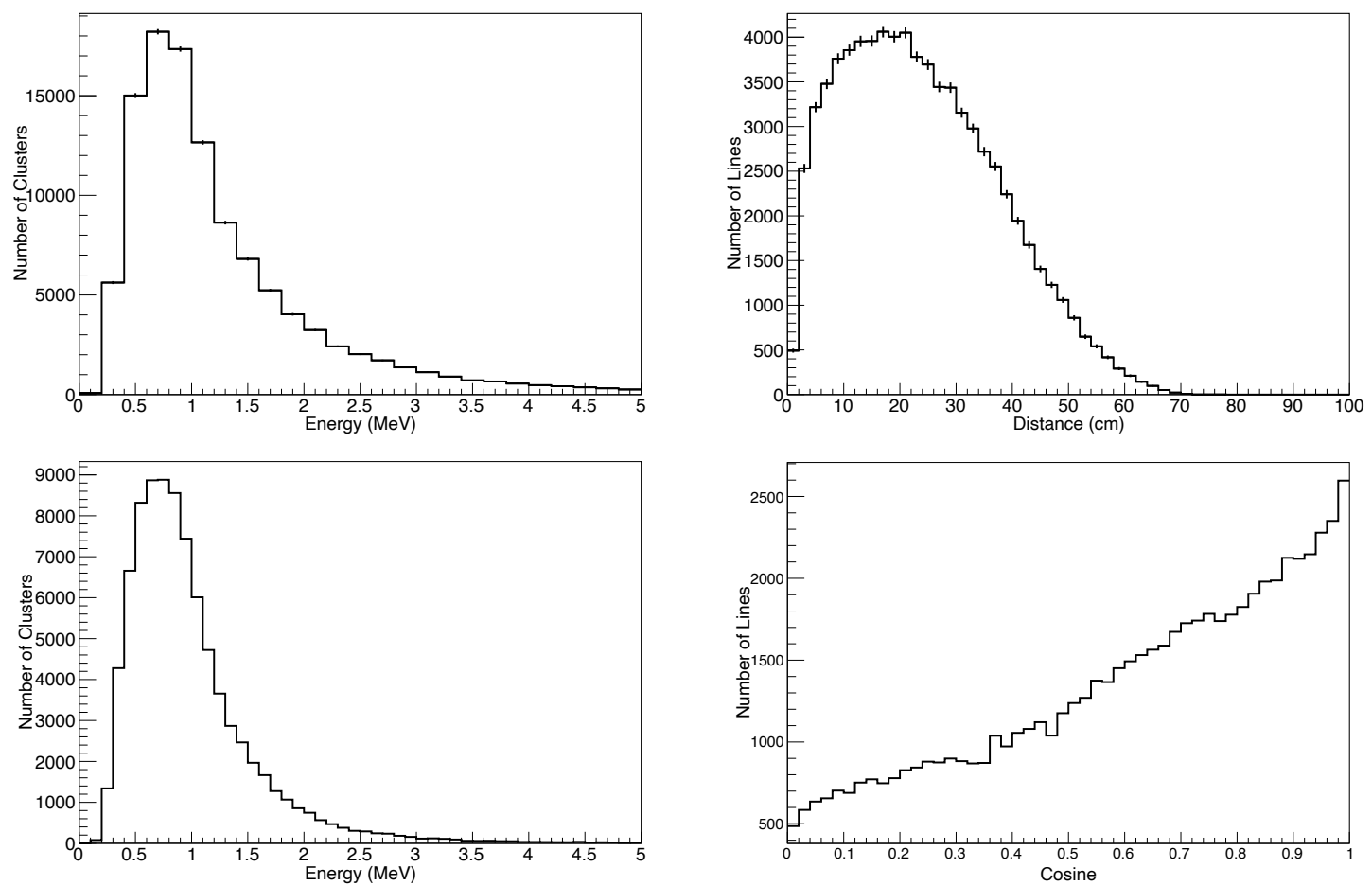

Figure 6.3. Top left: Energy deposited in each cluster in high-beam events with at least two clusters. Top right: Distance between clusters in a line in events with two or more clusters. Bottom left: Energy deposited in individual one-hit clusters for high beam events. The majority $(97 \%)$ are below $2.5 \mathrm{MeV}$. Bottom right: Cosine of angle between line and vector pointing to the target. Any mCP signal should appear at $\cos \theta=1$.

the vertical direction $(y)$ and $0.16 \mathrm{~cm}$ along the beam direction $(z)$ [15]. The smaller uncertainty in the drift direction compared to the other directions is due to the frequency of the detector readout, which samples the drift distance in $0.03 \mathrm{~cm}$ samples. The uncertainties in the other two directions depend on the wire spacing and orientation of the wire planes; thus the uncertainties in the beam and vertical directions are not the same. There is also a global uncertainty of $1.52 \mathrm{~cm}$ in the drift direction due to the $10 \mu \mathrm{s}$ beam spill window. This uncertainty in the arrival time of the beam has the same effect on both clusters in a line. While these uncertainties are small compared to the size of the detector, they can become quite large, depending on the relative location of the points, when extrapolated to the location of the target, 1033 $m$ upstream. Since we use the position of the intersection of the lines on the plane to 
identify signal events coming from the target, we want events with good directional resolution and thus place a cut of $>10 \mathrm{~cm}$ on the separation between clusters. For two clusters in the center of the detector and separated by $10 \mathrm{~cm}$, the uncertainty at the target plane is $41 \mathrm{~m}$ in the vertical and $2.25 \mathrm{~m}$ in the horizontal directions for lines that point in the vicinity of the target. By applying the $10 \mathrm{~cm}$ cut on the separation between the two clusters we are ensuring that the uncertainties at the target plane are always smaller than these. Events where the two clusters are separated by less than $0.4 \mathrm{~cm}$ in the beam $(z)$ direction are also ignored to remove lines with undefined slope.

The locations and the uncertainties of the points of intersection of the lines with the plane at the target's edge are shown in Figure 6.4, where the target is located at the center. Only points at a distance $<10(100) \mathrm{m}$ from the target in the horizontal (vertical) direction are shown in the figure. We note that double hit events that are separated in $y$ by less than the vertical resolution will always be reconstructed as horizontal in the lab frame due to the discrete nature of the detector wires. This feature, which is generic for a discrete detector, leads to a population of horizontally reconstructed events with $\mathrm{Y}=-61 \mathrm{~m}$ in Figure 6.4 because the beam is pointing $3^{\circ}$ downwards.

\subsection{Expected Signal and Background}

The number of expected background events is estimated using a Monte Carlo simulation, assuming that the lines are isotropic and taking the distribution of cluster separation from data, as shown in Figure 6.3 (bottom right). We estimate the probability that two clusters will align with the target within the uncertainties. With the detector performance parameters reported above, and taking into account the spatial separation of clusters and the resulting uncertainties, we expect 1.46 background events which point back to the target. 


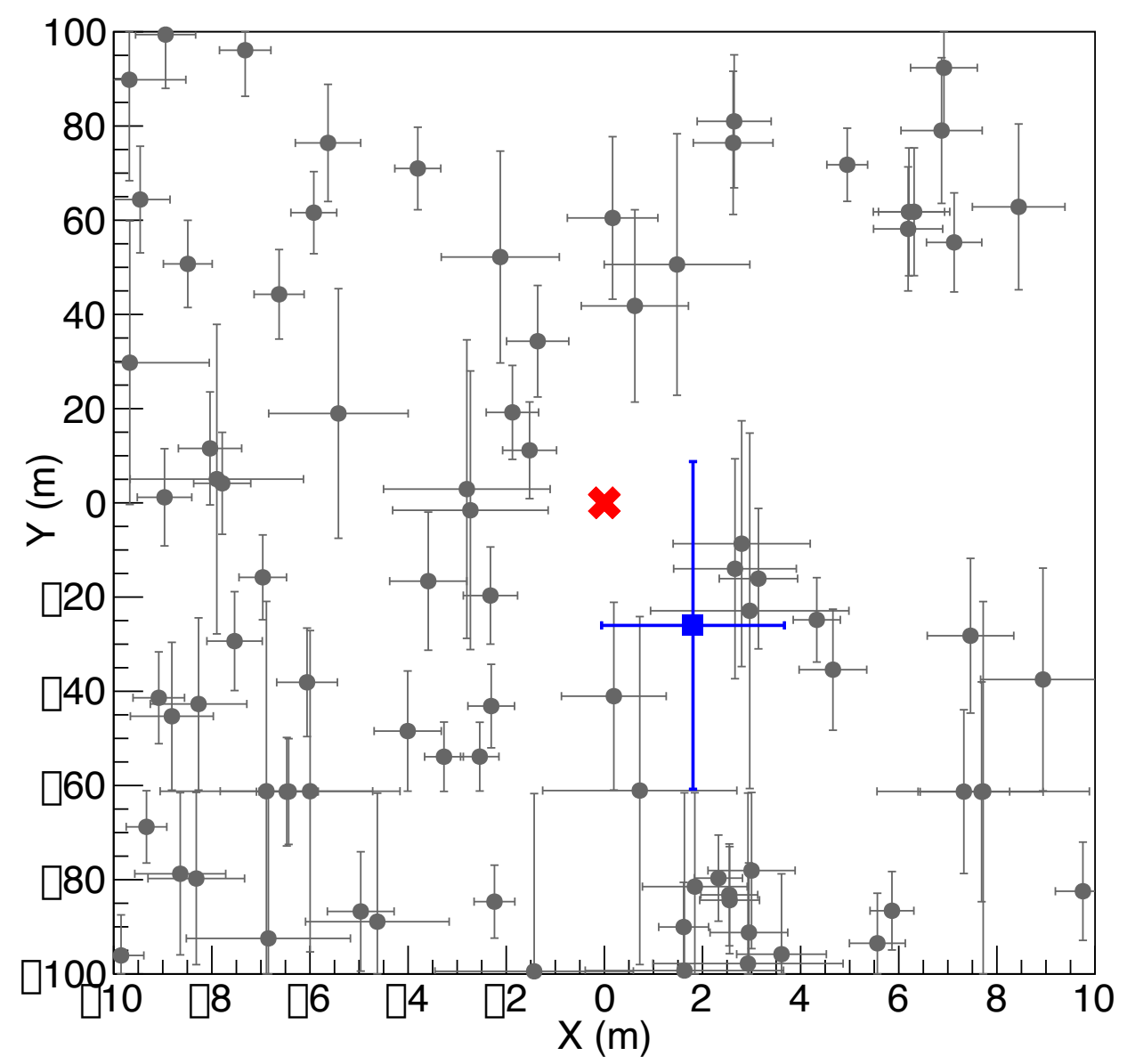

Figure 6.4. Locations of the points of intersections (shown as grey circles) of lines defined by two clusters with a plane perpendicular to the beam at the downstream target's edge. Uncertainties in the location (see text) are also shown. The target, denoted by the red cross, is located at $(0,0)$. The candidate signal event, denoted with a blue square, is consistent with originating from the target within its uncertainties. Note that the scale in the vertical axis is $10 \times$ that of the horizontal axis, since the horizontal uncertainties are smaller. Only points at a distance $<10(100)$ $\mathrm{m}$ from the target in the horizontal (vertical) direction are shown. 
The rate of expected mCPs passing through the ArgoNeuT detector depends on the mass of the mCP. The geometrical acceptance varies between $10^{-5}$ to $10^{-7}$ for signal events [21]. The detection probability for double-hit signals is inversely proportional to the fourth power of its electric charge $Q_{\chi}$.

The number of expected background events can be estimated by assuming the lines in double hit events are isotropic. The distribution of cluster separation is taken from data, shown in Figure 6.3 (top right), using pairs of events which do not point to the target's vicinity (cosine $>0$ in Fig. 6.3 (bottom right)). Using a Monte Carlo simulation, assuming that the lines are isotropic and taking the distribution of cluster separation from data, we estimate the probability that two clusters will align with the target within the uncertainties. With the detector performance parameters reported above, and taking into account the spatial separation of clusters and the resulting uncertainties, we expect 1.46 background events which point back to the target.

\subsection{Search for mCP Candidates}

We found one possible mCP signal candidate event, shown as a blue square in Figure 6.4. The position of the line in this event overlaps with the location of the target within the horizontal and vertical uncertainties. The event has been visually

scanned, and it shows no anomalies. The selected event, shown in Figure 6.5, has two clusters spaced $11.8 \mathrm{~cm}$ apart with with an energy of $0.72(2.82) \mathrm{MeV}$ in the more upstream (downstream) cluster. The observed candidate signal event is compatible with the expected background.

Before using this observation to set a limit, we consider the systematic uncertainty related to ArgoNeuT's exact orientation with respect to the target. Using the spread in direction of through-going muons [90], we find that the direction of the target location is uncertain by $\pm 1.0^{\circ}$ horizontally and $\pm 0.59^{\circ}$ vertically. In the plane 
of Figure 6.4, this corresponds to $\pm 18 \mathrm{~m}$ in $x$ and $\pm 10.6 \mathrm{~m}$ in $y$. When the target location is moved within this uncertainty window, up to five two-cluster events can be found in the ArgoNeuT high-beam data. We set limits using both one and five observed events and treat the difference as a systematic uncertainty. As an additional test, we have checked that the number of signal events in the plane of Figure 6.4 is consistent with a Poisson distribution as the target location is allowed to vary across a large window (well beyond the systematic uncertainty). We have also considered the effect of the mCPs traversing the dirt en route from the target to the detector, following [21]. We find that the amount of energy loss is negligible in the region of interest. The angular deflection of an $\mathrm{mCP}$ from elastic scattering off of nuclei is also negligible for most of our parameter space. The angular deflection may become of order the typical spatial resolution only for $\epsilon \gtrsim 10^{-1}$ and thus can affect the limit only for $m_{\chi}$ above $2 \mathrm{GeV}$. We estimate the limit for these high masses can be weaker by about $15 \%$ in $\epsilon$.

The expected number of mCPs traversing ArgoNeuT and their energy distribution for a given mCP mass and charge are simulated with Pythia 8 [91], as detailed in Ref. [21]. The mean free path for every mCP is computed through equation (3.3) following the procedure in [21], giving a probability to deposit a double hit event. We then set limits using a CLs method [1] without subtracting background. Figure 6.6 shows our limits on mCPs as a function of their mass and charge. We put constraints at the $95 \%$ confidence level on $\mathrm{mCP}$ parameters that do not produce more than 4.7 events for one observed signal event.

To account for the uncertainty in detector orientation discussed above, we also draw a limit on parameters that lead to more than 10.5 events, corresponding to five observed signal events, and draw a band between these two cases. We note that the limits in both these cases are very close. These upper limits on the number 
of expected events correspond to the conservative assumption that the background cannot be subtracted. The results of previous experiments [22-26] are shown for comparison. Our result is a significant increase in the exclusion region in the range of millicharged masses $>0.1 \mathrm{GeV}$ and charge $<10^{-1} e$.

\subsection{Summary}

We have set new constraints from a search for millicharged particles in the ArgoNeuT LArTPC experiment at Fermilab. For a detector exposure of $1.0 \times 10^{20}$ POT, one candidate event has been observed, compatible with the expected background. ArgoNeuT has probed the region of $Q_{\chi}=10^{-1} e-10^{-3} e$ for masses in the range $m_{\chi}=0.1-3 \mathrm{GeV}$, unexplored by previous experiments. This analysis represents the first search for millicharged particles in a LArTPC neutrino detector, performed with a novel search method using a cluster doublet aligned with the beam target location. The analysis techniques used in this search can be applied to future larger mass LArTPC experiments and motivate new searches. 

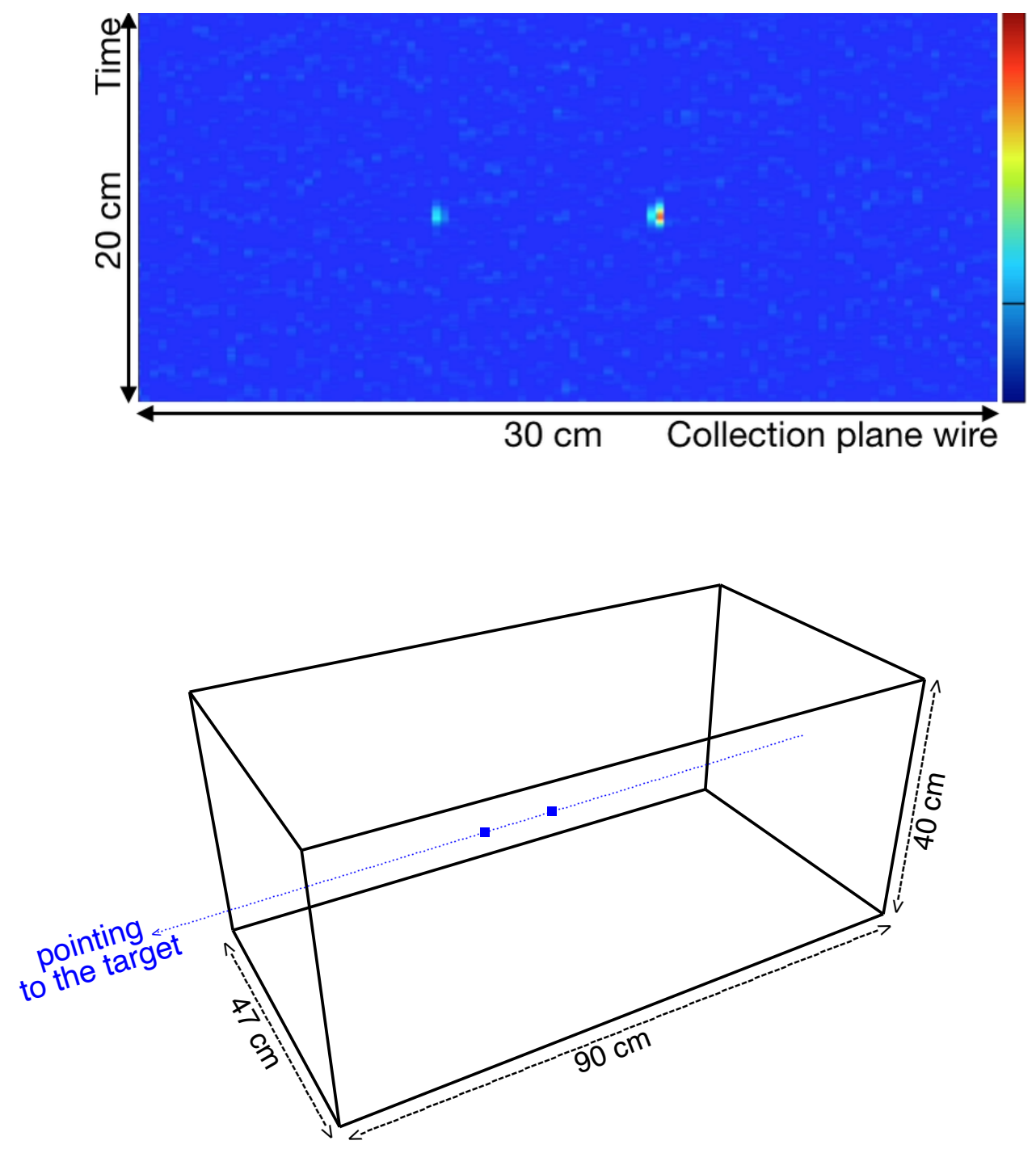

Figure 6.5. The candidate signal event. Top: Zoomed in image from the collection wire plane. Two isolated clusters are visible in the event. The two clusters are spaced $11.8 \mathrm{~cm}$ apart with an energy of $0.72(2.82) \mathrm{MeV}$ in the left (right) cluster. Color in the image indicates the amount of charge collected. The horizontal axis is perpendicular to the collection plane wires. The vertical axis is parallel to the drift direction. Bottom: 3D reconstruction of the event with the reconstructed line superimposed. 


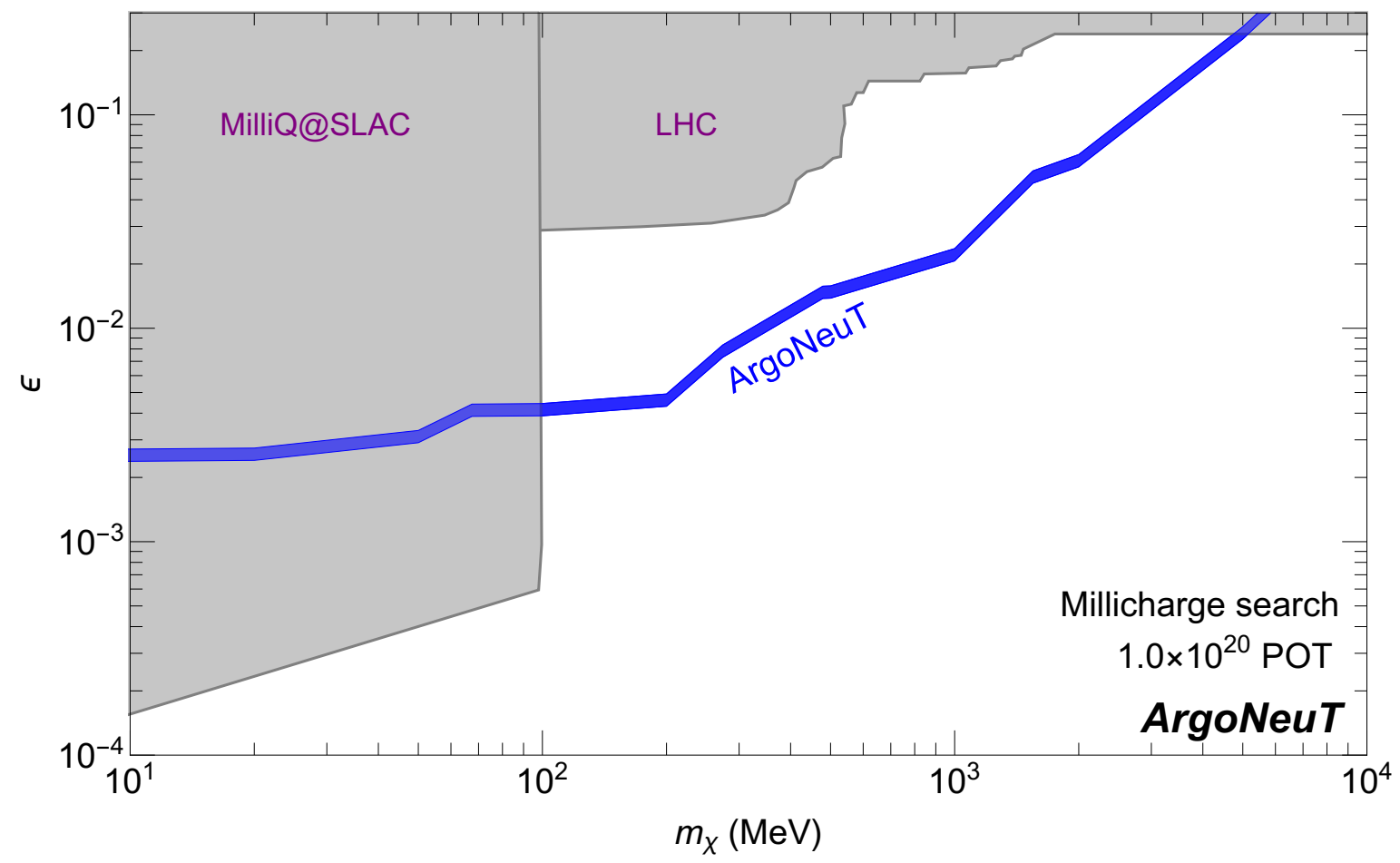

Figure 6.6. ArgoNeuT limits (blue) in the $m_{\chi}-\epsilon$ plane for millicharged particles at $95 \%$ C.L., where $\epsilon \equiv Q_{\chi} / e$. The limit is drawn where $\mathrm{mCP}$ are unlikely to produce more than the observed number of events. The thickness of the blue band accounts for the systematic uncertainty in detector placement. Existing limits from other experiments, including SLAC MilliQ [22] and collider experiments [23-26], are shown in gray. 


\section{CHAPTER 7 CONCLUSIONS}

This thesis has demonstrated the capability of liquid argon time projection chambers to detect sub-MeV-scale energy depositions. Two important results are presented. They are the first physics measurements in this energy range in a LArTPC. In Chapter 5, we presented a study of low-energy photons produced in neutrino interactions. Using data from ArgoNeuT, MeV-scale energy depositions from photons from nuclear deexcitation and inelastic neutron scattering were identified and reconstructed, and a novel reconstruction technique has been developed. The reconstructed number, energies and positions of these depositions were compared to a FLUKA simulation of neutrino-argon interactions. The reconstructed activity is consistent with that created by photons produced when the final-state nucleus deexcites and by photons produced by inelastic scattering of final-state neutrons. This study demonstrated that reconstruction at $\mathrm{MeV}$ scales in LArTPCs is indeed possible. It also shows that existing neutrino generators are capable of correctly simulating final state photon and neutron products in neutrino-argon interactions.

Mev-scale reconstruction capabilities are critically important for future lowenergy LArTPC studies. For supernova and solar neutrinos, MeV-scale feature reconstruction may enable enhanced energy reconstruction and the ability to differentiate between interaction channels, greatly enhancing the versatility of these datasets to answer key questions in particle and astrophysics. This study indicates that for $\mathrm{GeV}$-scale neutrinos, reconstruction of the energies and multiplicities of deexcitation photons and final-state neutrons may very well be possible in large LArTPCs, such as DUNE. The ability to accurately reconstruct these two neutral particles aids greatly in the reconstruction of beam neutrino energies and the final state nucleus. This 
in turn may provide insight into the differences between neutrino and antineutrino interactions in these experiments.

This work has already influenced several theoretical studies discussing the potential of other LArTPCs, particularly DUNE. These studies have discussed the resolution of LArTPCs [78] and the ability for DUNE to study solar neutrinos [19]. A follow-up analysis mimicking the techniques developed here is currently being performed in MicroBooNE in the hopes of increasing the statistical power of the results presented in this thesis.

This thesis also includes the first search for beyond the Standard Model physics in a LArTPC, a search for millicharged particles in data from ArgoNeuT presented in Chapter 6. This search used the low-energy techniques developed in the previous analysis to search for isolated low-energy depositions from millicharged particles. This search sets world-leading limits on the parameter space of millicharged particles. This search also demonstrates the potential of LArTPCs in BSM physics studies and shows that even a small LArTPC can set limits competitive with dedicated millicharged particle experiments and large experiments, like those at the Large Hadron Collider.

The first analysis is published in Physical Review D [82] and presented at several conferences, including a Joint Experimental-Theoretical Physics Seminar at Fermilab. The second analysis has been posted on the arXiv [89] and submitted to Physical Review Letters, in addition to being presented at conferences. 


\section{BIBLIOGRAPHY}

[1] M. Tanabashi et al., "Review of particle physics," Phys. Rev. D, vol. 98, p. 030001, Aug 2018.

[2] D. Griffiths, Introduction to Elementary Particles. Weinheim, Germany: Wiley, 2008.

[3] R. Acciarri et al., "Long-Baseline Neutrino Facility (LBNF) and Deep Underground Neutrino Experiment (DUNE)," 2015. arXiv:1512.06148.

[4] D. Lincoln, "The standard model: The most successful theory ever." Online at http://www.fnal.gov/pub/today/archive/archive_2011/today11-1118_NutshellStandardModelReadMore.html.

[5] J. A. Formaggio and G. P. Zeller, "From eV to EeV: Neutrino cross sections across energy scales," Rev. Mod. Phys., vol. 84, pp. 1307-1341, 2012.

[6] P. Adamson et al., "The NuMI neutrino beam," Nucl. Instrum. Meth., vol. A806, pp. 279-306, 2016.

[7] R. Acciarri et al., "First observation of low energy electron neutrinos in a liquid argon time projection chamber," Phys. Rev., vol. D95, no. 7, p. 072005, 2017.

[8] A. A. Aguilar-Arevalo et al., "The neutrino flux prediction at MiniBooNE," Phys. Rev., vol. D79, p. 072002, 2009.

[9] J. N. Bahcall, A. M. Serenelli, and S. Basu, "New solar opacities, abundances, helioseismology, and neutrino fluxes," Astrophys. J., vol. 621, pp. L85-L88, 2005.

[10] K. Scholberg, "Supernova neutrino detection," Nucl. Phys. Proc. Suppl., vol. 91, pp. 331-337, 2001.

[11] K. Zuber, Neutrino Physics, Second Edition. Series in High Energy Physics, Cosmology and Gravitation, Boca Raton, FL: Taylor \& Francis, 2011.

[12] R. Acciarri et al., "Michel electron reconstruction using cosmic-ray data from the MicroBooNE LArTPC," JINST, vol. 12, no. 09, p. P09014, 2017.

[13] XCOM: Photon Cross Section Database. (Version 1.5), National Institute of Standards and Technology. Accessed: February 8, 2018. [Online]. Available: http://physics.nist.gov/xcom.

[14] N. Otuka et al., "Towards a more complete and accurate experimental nuclear reaction data library (EXFOR): International collaboration between nuclear reaction data centres (NRDC)," Nucl. Data Sheets, vol. 120, pp. 272-276, 2014.

[15] C. Anderson et al., "The ArgoNeuT detector in the NuMI low-energy beam line at Fermilab," JINST, vol. 7, p. P10019, 2012.

[16] R. Acciarri et al., "A study of electron recombination using highly ionizing particles in the ArgoNeuT Liquid Argon TPC," JINST, vol. 8, p. P08005, 2013.

[17] R. Acciarri et al., "Detection of back-to-back proton pairs in charged-current neutrino interactions with the ArgoNeuT detector in the NuMI low energy beam line," Phys. Rev., vol. D90, no. 1, p. 012008, 2014. 
[18] M. Elkins et al., "Neutron measurements from antineutrino hydrocarbon reactions," Phys. Rev., vol. D100, no. 5, p. 052002, 2019.

[19] F. Capozzi, S. W. Li, G. Zhu, and J. F. Beacom, "DUNE as the next-generation solar neutrino experiment," Phys. Rev. Lett., vol. 123, no. 13, p. 131803, 2019.

[20] ESTAR, PSTAR, and ASTAR: Computer Programs for Calculating StoppingPower and Range Tables for Electrons, Protons, and Helium Ions. (Version 1.2.3), National Institute of Standards and Technology. Accessed: December 8, 2017. [Online]. Available: http://physics.nist.gov/Star.

[21] R. Harnik, Z. Liu, and O. Palamara, "Millicharged particles in liquid argon neutrino experiments," JHEP, vol. 07, p. 170, 2019.

[22] A. A. Prinz et al., "Search for millicharged particles at SLAC," Phys. Rev. Lett., vol. 81, pp. 1175-1178, 1998.

[23] H. Vogel and J. Redondo, "Dark Radiation constraints on minicharged particles in models with a hidden photon," JCAP, vol. 1402, p. 029, 2014.

[24] R. Essig et al., "Working Group Report: New Light Weakly Coupled Particles," in Proceedings, 2013 Community Summer Study on the Future of U.S. Particle Physics: Snowmass on the Mississippi (CSS2013): Minneapolis, MN, USA, July 29-August 6, 2013, 2013.

[25] S. Chatrchyan et al., "Search for fractionally charged particles in $p p$ collisions at $\sqrt{s}=7$ TeV," Phys. Rev., vol. D87, no. 9, p. 092008, 2013.

[26] J. Jaeckel, M. Jankowiak, and M. Spannowsky, "LHC probes the hidden sector," Phys. Dark Univ., vol. 2, pp. 111-117, 2013.

[27] C. Adams et al., "The Long-Baseline Neutrino Experiment: Exploring fundamental symmetries of the universe," in Snowmass 2013: Workshop on Energy Frontier Seattle, USA, June 30-July 3, 2013, 2013. arXiv:1307.7335.

[28] M. Antonello et al., "A proposal for a three detector Short-Baseline Neutrino Oscillation Program in the Fermilab Booster Neutrino Beam," 2015. arXiv:1503.01520.

[29] C. Giunti and C. Kim, Fundamentals of Neutrino Physics and Astrophysics. New York, NY: Oxford University Press, 2007.

[30] E. Fermi, "An attempt of a theory of beta radiation," Z. Phys., vol. 88, pp. 161$177,1934$.

[31] C. L. Cowan, F. Reines, F. B. Harrison, H. W. Kruse, and A. D. McGuire, "Detection of the free neutrino: A confirmation," Science, vol. 124, pp. 103-104, 1956.

[32] G. Danby, J. M. Gaillard, K. A. Goulianos, L. M. Lederman, N. B. Mistry, M. Schwartz, and J. Steinberger, "Observation of high-energy neutrino reactions and the existence of two kinds of neutrinos," Phys. Rev. Lett., vol. 9, pp. 36-44, 1962.

[33] F. Suekane, Neutrino Oscillations: A Practical Guide to Basics and Applications. Lecture Notes in Physics, Tokyo, Japan: Springer, 2015. 
[34] K. Kodama et al., "Observation of tau neutrino interactions," Phys. Lett., vol. B504, pp. 218-224, 2001.

[35] V. N. Gribov and B. Pontecorvo, "Neutrino astronomy and lepton charge," Phys. Lett., vol. 28B, p. 493, 1969.

[36] Q. R. Ahmad et al., "Measurement of the rate of $\nu_{e}+d \rightarrow p+p+e^{-}$interactions produced by ${ }^{8} B$ solar neutrinos at the Sudbury Neutrino Observatory," Phys. Rev. Lett., vol. 87, p. 071301, 2001.

[37] Y. Fukuda et al., "Evidence for oscillation of atmospheric neutrinos," Phys. Rev. Lett., vol. 81, pp. 1562-1567, 1998.

[38] M. H. Ahn et al., "Indications of neutrino oscillation in a $250 \mathrm{~km}$ long baseline experiment," Phys. Rev. Lett., vol. 90, p. 041801, 2003.

[39] D. G. Michael et al., "Observation of muon neutrino disappearance with the MINOS detectors and the NuMI neutrino beam," Phys. Rev. Lett., vol. 97, p. 191801, 2006.

[40] N. Agafonova et al., "Observation of a first $\nu_{\tau}$ candidate in the OPERA experiment in the CNGS beam," Phys. Lett., vol. B691, pp. 138-145, 2010.

[41] K. Abe et al., "Indication of electron neutrino appearance from an acceleratorproduced off-axis muon neutrino beam," Phys. Rev. Lett., vol. 107, p. 041801, 2011.

[42] D. G. Michael et al., "The Magnetized steel and scintillator calorimeters of the MINOS experiment," Nucl. Instrum. Meth., vol. A596, pp. 190-228, 2008.

[43] J. Bahcall, Neutrino Astrophysics. Cambridge, UK: Cambridge University Press, 1989.

[44] A. Phillips, The Physics of Stars. West Sussex, UK: Wiley, 1994.

[45] G. Bellini et al., "Final results of Borexino Phase-I on low energy solar neutrino spectroscopy," Phys. Rev., vol. D89, no. 11, p. 112007, 2014.

[46] S. P. Mikheev and A. Yu. Smirnov, "Resonant amplification of neutrino oscillations in matter and solar neutrino spectroscopy," Nuovo Cim., vol. C9, pp. 17-26, 1986.

[47] R. Fardon, A. E. Nelson, and N. Weiner, "Dark energy from mass varying neutrinos," JCAP, vol. 0410, p. 005, 2004.

[48] M. Longair, High Energy Astrophysics. Cambridge, UK: Cambridge University Press, 2011.

[49] H. A. Bethe, "Supernova mechanisms," Rev. Mod. Phys., vol. 62, pp. 801-866, 1990.

[50] A. Friedland and A. Gruzinov, "Neutrino signatures of supernova turbulence," 2006. arXiv:astro-ph/0607244.

[51] R. C. Schirato and G. M. Fuller, "Connection between supernova shocks, flavor transformation, and the neutrino signal," 2002. arXiv:astro-ph/0205390. 
[52] F. Hanke et al., "SASI activity in three-dimensional neutrino-hydrodynamics simulations of supernova cores," Astrophys. J., vol. 770, p. 66, 2013.

[53] K. Winter, T. Ericson, and P. Landshoff, Neutrino Physics. Cambridge Monographs on Particle Physics, Nuclear Physics and Cosmology, Cambridge, UK: Cambridge University Press, 2000.

[54] F. P. An et al., "Observation of electron-antineutrino disappearance at Daya Bay," Phys. Rev. Lett., vol. 108, p. 171803, 2012.

[55] J. Ashenfelter et al., "First search for short-baseline neutrino oscillations at HFIR with PROSPECT," Phys. Rev. Lett., vol. 121, no. 25, p. 251802, 2018.

[56] K. N. Abazajian et al., "Light sterile neutrinos: A white paper," 2012. arXiv:1204.5379.

[57] F. Boehm and P. Vogel, Physics of Massive Neutrinos. Cambridge, UK: Cambridge University Press, 1992.

[58] D. S. Ayres et al., "The NOvA technical design report," tech. rep., Fermilab., Batavia, IL, 2007. Technical Report Number FERMILAB-DESIGN-2007-01.

[59] J. Ashenfelter et al., "The PROSPECT reactor antineutrino experiment," Nucl. Instrum. Meth., vol. A922, pp. 287-309, 2019.

[60] S. N. Gninenko, D. V. Kirpichnikov, and N. V. Krasnikov, "Probing millicharged particles with NA64 experiment at CERN," Phys. Rev., vol. D100, no. 3, p. 035003, 2019.

[61] A. o. Berlin, "Severely constraining dark matter interpretations of the 21-cm anomaly," Phys. Rev. Lett., vol. 121, no. 1, p. 011102, 2018.

[62] G. Magill et al., "Millicharged particles in neutrino experiments," Phys. Rev. Lett., vol. 122, no. 7, p. 071801, 2019.

[63] K. J. Kelly and Y.-D. Tsai, "Proton fixed-target scintillation experiment to search for millicharged dark matter," Phys. Rev., vol. D100, no. 1, p. 015043 , 2019

[64] W. Leo, Techniques for Nuclear and Particle Physics Experiments: A How-to Approach. Berlin, Germany: Springer, 2012.

[65] B. Abi et al., "The single-phase ProtoDUNE technical design report," 2017. arXiv:1706.07081.

[66] C. Rubbia et al., "Underground operation of the ICARUS T600 LAr-TPC: first results," JINST, vol. 6, p. P07011, 2011.

[67] P. Adamson et al., "LArIAT: Liquid Argon TPC in a Test Beam," tech. rep., Fermilab., Batavia, IL, 2013. Tecnhical Report Number FERMILAB-PROPOSAL1034 .

[68] D. J. Hughes, Neutron Cross Sections. London, UK: Pergamon Press, 1957.

[69] R. Acciarri et al., "Measurements of inclusive muon neutrino and antineutrino charged current differential cross sections on argon in the NuMI antineutrino beam," Phys. Rev., vol. D89, no. 11, p. 112003, 2014. 
[70] R. Acciarri et al., "Design and construction of the MicroBooNE detector," JINST, vol. 12, no. 02, p. P02017, 2017.

[71] C. Adams et al., "Design and construction of the MicroBooNE cosmic ray tagger system," JINST, vol. 14, no. 04, p. P04004, 2019.

[72] E. L. Snider and G. Petrillo, "LArSoft: toolkit for simulation, reconstruction and analysis of liquid argon TPC neutrino detectors," J. Phys. Conf. Ser., vol. 898, no. 4, p. 042057, 2017.

[73] R. Acciarri et al., "First measurement of the cross section for $\nu_{\mu}$ and $\overline{\nu_{\mu}}$ induced single charged pion production on argon using ArgoNeuT," Phys. Rev., vol. D98, no. 5 , p. $052002,2018$.

[74] R. Acciarri et al., "First measurement of neutrino and antineutrino coherent charged pion production on argon," Phys. Rev. Lett., vol. 113, no. 26, p. 261801, 2014.

[75] P. Abratenko et al., "First measurement of inclusive muon neutrino charged current differential cross sections on argon at $E_{\nu}=0.8 \mathrm{GeV}$ with the MicroBooNE detector," Phys. Rev. Lett., vol. 123, no. 13, p. 131801, 2019.

[76] C. Adams et al., "Comparison of $\nu_{\mu}$-Ar multiplicity distributions observed by MicroBooNE to GENIE model predictions," Eur. Phys. J., vol. C79, no. 3, p. 248, 2019.

[77] M. Antonello et al., "Search for anomalies in the $\nu_{e}$ appearance from a $\nu_{\mu}$ beam," Eur.Phys.J., vol. C73, p. 2345, 2013.

[78] A. Friedland and S. W. Li, "Understanding the energy resolution of liquid argon neutrino detectors," Phys. Rev., vol. D99, no. 3, p. 036009, 2019.

[79] G. Zhu, S. W. Li, and J. F. Beacom, "Developing the MeV potential of DUNE: Detailed considerations of muon-induced spallation and other backgrounds," Phys. Rev., vol. C99, no. 5, p. 055810, 2019.

[80] C. Grant and B. Littlejohn, "Opportunities with decay-at-rest neutrinos from decay-in-flight neutrino beams," PoS, vol. ICHEP2016, p. 483, 2016.

[81] P. A. Machado, O. Palamara, and D. W. Schmitz, "The Short-Baseline Neutrino Program at Fermilab," Ann. Rev. Nucl. Part. Sci., vol. 69, 2019.

[82] R. Acciarri et al., "Demonstration of MeV-scale physics in liquid argon time projection chambers using ArgoNeuT," Phys. Rev., vol. D99, no. 1, p. 012002, 2019 .

[83] A. Ferrari et al., "FLUKA: A multi-particle transport code (Program version 2005)," tech. rep., CERN., Geneva, Switzerland, 2005. Technical Report Numbers CERN-2005-010, SLAC-R-773, INFN-TC-05-11.

[84] Chart of Nuclides Database, National Nuclear Data Center, December 8, 2017. [Online]. Available: http://www.nndc.bnl.gov/chart/.

[85] O. Palamara, "Exclusive muon neutrino charged current pion-less topologies. ArgoNeuT results and future prospects in LAr TPC detectors," JPS Conf. Proc., vol. 12, p. 010017, 2016. 
[86] C. Andreopoulos et al., "The GENIE neutrino Monte Carlo generator," Nucl. Instrum. Meth., vol. A614, pp. 87-104, 2010.

[87] S. N. Gninenko, "New limits on radiative sterile neutrino decays from a search for single photons in neutrino interactions," Phys. Lett., vol. B710, pp. 86-90, 2012.

[88] E. Bertuzzo et al., "Neutrino Masses and Mixings Dynamically Generated by a Light Dark Sector," Phys. Lett., vol. B791, pp. 210-214, 2019.

[89] R. Acciarri et al., "Improved limits on millicharged particles using the ArgoNeuT experiment at Fermilab," 2019. arXiv:1911.07996.

[90] C. Anderson et al., "Analysis of a large sample of neutrino-induced muons with the ArgoNeuT detector," JINST, vol. 7, p. P10020, 2012.

[91] T. Sjostrand, S. Mrenna, and P. Z. Skands, "A brief introduction to PYTHIA 8.1," Comput. Phys. Commun., vol. 178, pp. 852-867, 2008. 